\section{Karl-Friedrich Böhringer}

University of Washington, Seattle

Department of Electrical Engineering

234 EE/CSE Building

Box 352500

Seattle, WA 98195-2500

karl@ee.washington.edu

www.ee.washington.edu/faculty/karl

\section{Bruce Randall Donald}

Dartmouth College

Department of Computer Science

6211 Sudikoff Laboratory

Hanover, New Hampshire 03755-3510, USA

brd@cs.darmouth.edu www.cs.dartmouth.edu/ ₹brd

\section{Noel C. MacDonald}

Cornell University

Department of Electrical Engineering and

Cornell Nanofabrication Facility

408 Phillips Hall

Ithaca, New York 14853, USA

mmacd@ee.cornell.edu

www.engr.cornell.edu/ee/MacDonald.html

\title{
Programmable Force Fields for Distributed Manipulation, with Applications to MEMS Actuator Arrays and Vibratory Parts Feeders*
}

\begin{abstract}
Programmable force vector fields can be used to control a variety of flexible planar parts feeders such as massively parallel microactuator arrays or transversely vibrating (macroscopic) plates. These new automation designs promise great flexibility, speed, and dexteritywe believe they may be employed to position, orient, singulate, sort, feed, and assemble parts. However, since they have only recently been invented, programming and controlling them for manipulation tasks is challenging. When a part is placed on our devices, the programmed vector field induces a force and moment upon it. Over time, the part may come to rest in a dynamic equilibrium state. By chaining sequences of force fields, the equilibrium states of a part in the field may be cascaded to obtain a desired final state. The resulting strategies require no sensing, and enjoy efficient planning algorithms.

This paper begins by describing new experimental devices that can implement programmable force fields. In particular, we describe our progress in building the M-CHIP (Manipulation CHIP), a massively parallel array of programmable micromotion pixels. Both the M-CHIP and other microarray devices, as well as macroscopic devices such as transversely vibrating plates, may be programmed
\end{abstract}

\footnotetext{
*An earlier, much shorter version of this paper was presented at the Workshop on Algorithmic Foundations of Robotics (Toulouse, France, July 1996). The International Journal of Robotics Research Vol. 18, No. 2, February 1999, pp. xxx-xxx, (C)1999 Sage Publications, Inc.
}

with vector fields, and their behavior predicted and controlled using our equilibrium analysis. We demonstrate lower bounds (i.e., impossibility results) on what the devices cannot do, and results on a classification of control strategies yielding design criteria by which well-behaved manipulation strategies may be developed. We provide sufficient conditions for programmable fields to induce well-behaved equilibria on every part placed on our devices. We define composition operators to build complex strategies from simple ones, and show the resulting fields are also well behaved. We discuss whether fields outside this class can be useful and free of pathology.

Using these tools, we describe new manipulation algorithms. In particular, we improve existing planning algorithms by a quadratic factor, and the plan length by a linear factor. Using our new and improved strategies, we show how to simultaneously orient and pose any part, without sensing, from an arbitrary initial configuration. We relax earlier dynamic and mechanical assumptions to obtain more robust and flexible strategies.

Finally, we consider parts feeders that can only implement a very limited "vocabulary" of vector fields (as opposed to the pixel-wise programmability assumed above). We show how to plan and execute parts posing and orienting strategies for these devices, but with a significant increase in planning complexity and some sacrifice in completeness guarantees. We discuss the trade-off between mechanical complexity and planning complexity. 


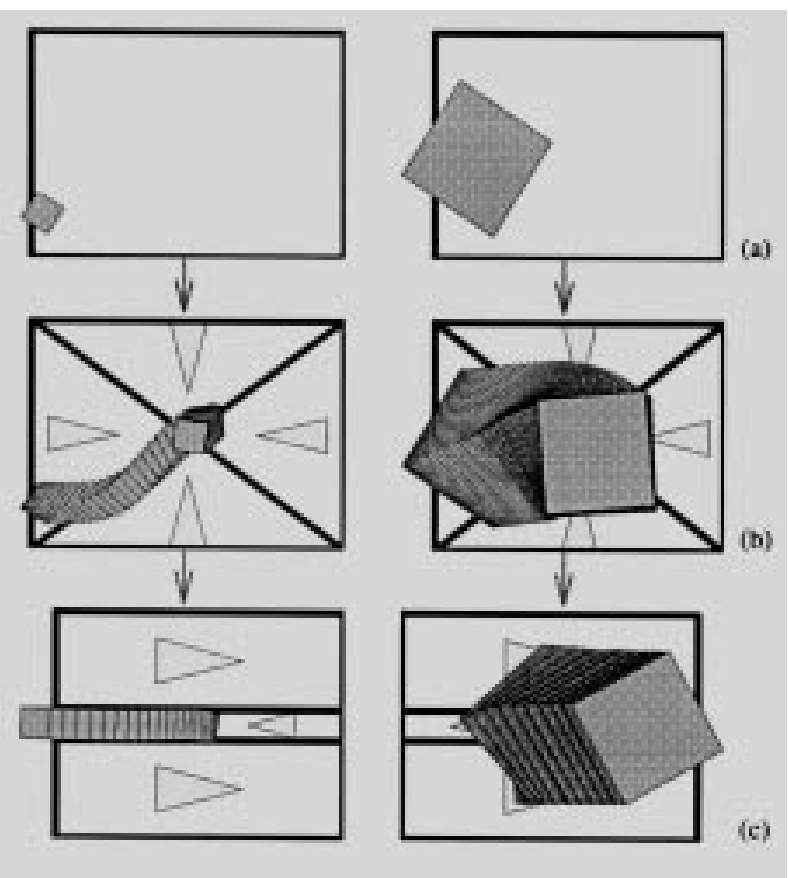

Fig. 1. Sensorless sorting using force vector fields: parts of different sizes are first centered, then subsequently separated, depending on their size.

\section{Introduction}

Programmable force fields offer a fundamentally new approach to automated parts manipulation. Instead of handling a part directly (e.g., with a robot gripper), a force field surrounding the part causes it to move. Programmable force fields promise great flexibility, speed, and dexterity for a wide variety of tasks such as parts orienting, positioning, singulating, sorting, feeding, and assembly. Recently, several devices have been invented that can implement programmable force fields: in particular, actuator arrays fabricated with Micro Electro Mechanical System (MEMS) technology, as well as macroscopic vibrating plates. These new automation designs permit distributed, parallel, nonprehensile, sensorless manipulation tasks that make them particularly attractive for handling batch microfabricated parts, whose small dimensions and large numbers would prohibit conventional pickand-place operations.

A wealth of geometric and algorithmic problems arise in the control and programming of manipulation systems with many independent actuators. The theory of programmable force fields represents the first systematic, computational attack on massively parallel distributed manipulation based on geometric and physical reasoning. The goal of this paper is to develop a science base for manipulation using programmable force fields, and to demonstrate experiments with prototype devices that support this theory. We present combinatorially precise planning algorithms that synthesize strategies for con- trolling and coordinating a very large number of distributed actuators in a principled, task-level fashion.

When a part is placed on such a device, the programmed vector field induces a force and moment upon it. Over time, the part may come to rest in a dynamic equilibrium state. In principle, we have tremendous flexibility in choosing the vector field, since using, e.g., MEMS array technologies, the force field may be programmed pixel-wise. Hence, we have a lot of control over the resulting equilibrium states. By chaining sequences of vector fields, the equilibria may be cascaded to obtain a desired final state-for example, this state may represent a unique orientation or pose of the part. A system with such a behavior exhibits the feeding property (Akella et al. 1995):

A system has the feeding property over a set of parts $\mathcal{P}$ and a set of initial configurations $\mathcal{I}$ if, given any part $P \in \mathcal{P}$, there is some output configuration $\mathbf{q}$ such that the system can move $P$ to $\mathbf{q}$ from any location in $\mathcal{X}$.

Our work on programmable vector fields is related to nonprehensile manipulation [Donald, Jennings, and Rus 1995; Zumel and Erdmann 1996; Erdmann and Mason 1996; Erdmann 1996]: in both cases, parts are manipulated without form or force closure.

This paper describes our experimental devices, a technique for analyzing them called equilibrium analysis, lower bounds (i.e., impossibility results) on what the devices cannot do, and results of a classification of control strategies yielding design criteria for useful manipulation strategies. Then we describe new manipulation algorithms using these tools. In particular, we improve earlier planning algorithms by a quadratic factor, show how to simultaneously orient and pose a part, and relax dynamic and mechanical assumptions to obtain more robust and flexible strategies.

One corollary of our results is a method for coordinating the actions of a large distributed actuation system. Such systems comprise arrays with up to tens of thousands of independently servoable actuator cells, which we call motion pixels. We show how these systems can be programmed in a fine-grained, SIMD (single instruction multiple data) fashion to exert force fields on the manipulated object, thereby accomplishing massively parallel distributed manipulation. Moreover, the theory of programmable force fields gives a method for controlling a large number of distributed actuators in a principled, geometric, task-level fashion. Whereas many control theories for multiple independent actuators break down as the number of actuators becomes large, our systems should only become more robust as the actuators become denser and more numerous.

The theory developed in this paper is applicable to any controllable array capable of generating force vector fields, and it is independent of the specific device hardware. We have tested it thoroughly in collaboration with J. Suh and G. Kovacs on a MEMS actuator array developed at Stanford (Böhringer et al. 1997c). This microcilia device consists of a $16 \times 16$ array of 
motion pixels, which covers an area of about $2 \mathrm{~cm} \times 2 \mathrm{~cm}$. Each pixel consists of four thermobimorph actuators. Actuators in each direction can be controlled independently by a graphical user interface on a personal computer. Böhringer and coworkers (1997b) reported on experiments in sensorless manipulation with the microcilia device. Small chips were placed at arbitrary initial positions on the array and were translated, rotated, centered, and aligned by the array without sensor feedback. These experiments constitute strong evidence in support of our theory of sensorless manipulation.

In this paper, we focus on the theoretical foundations of manipulation with programmable force fields. We pose the question, Which force fields are suitable for manipulation strategies? In particular, we ask whether the fields may be classified. That is, can we characterize all those force fields in which every part has stable equilibria? While this question has been well studied for a point mass in a field, the issue is more subtle when lifted to a body with finite area, due to the moment covector. To answer, we first demonstrate impossibility results, in the form of "lower bounds": there exist perfectly plausible fields that induce no stable equilibrium in simple parts.

Fortunately, there is also good news. We present conditions for fields to induce well-behaved equilibria,

by exploiting the theory of potential fields. While potential fields have been widely used in robot control (Khatib 1986; Koditschek and Rimon 1988; Rimon and Koditschek 1992; Reif and Wang 1995], microactuator arrays present us with the ability to explicitly program the applied force at every point in a vector field. Whereas previous work has developed control strategies with artificial potential fields, our fields are nonartificial (i.e., physical). Artificial potential fields require a tight feedback loop, in which at each clock tick, the robot senses its state and looks up a control (i.e., a vector) using a state-indexed navigation function (i.e., a vector field). In contrast, physical potential fields employ no sensing, and the motion of the manipulated object evolves in an open-loop manner (for example, like a particle in a gravity field). This alone makes our application of potential-field theory to microdevices unique and novel. Moreover, such fields can be composed using addition, sequential composition, "parallel" composition by superposition of controls, or by a new kind of "morphing" of control signals, which we will define.

Previous results on array manipulation strategies may be formalized using equilibrium analysis. Böhringer and colleagues proposed a family of control strategies called squeeze patterns, and a planning algorithm for parts orientation. This first result proved an $O\left(n^{2}\right)$ upper bound on the number $E$ of orientation equilibria of a nonpathological (see Section 3.2) planar part with $n$ vertices. This yields an $O\left(E^{2}\right)=O\left(n^{4}\right)$ planning algorithm to uniquely orient a part, under certain geometric, dynamic, and mechanical assumptions. In this paper, we argue that this bound on equilibria appears tight. This results in a high planning and execution complexity.
Using our equilibrium analysis, we introduce radial fields, which satisfy our stability property. Radial fields can then be combined with squeeze fields. We show this has several benefits:

1. the number of equilibria drops to $E=O(n)$;

2. the planning complexity drops to $O\left(E^{2}\right)=O\left(n^{2}\right)$;

3 . throughout the strategy execution, every part rotates about one fixed, unique point (after the first step); and

4. this means that we can dispense with one critical assumption (called 2PHASE by Böhringer and coworkers (1994a)): we no longer need to assume that the translational and rotational motions induced by the array interact in a "quasi-static" and "sequential" manner.

We motivate our results by beginning with a description of the experimental devices we are interested in programming. In particular, we describe our progress in building the M-CHIP (Manipulation CHIP), a massively parallel array of programmable micromotion pixels. As proof of the concept, we demonstrate a prototype M-CHIP containing up to 15,000 silicon actuators in $1 \mathrm{in}^{2}$. Our strategies are also applicable to macroscopic partsfeeders. We describe a planar, vibratory orienting and manipulation device that also uses our novel strategies.

Both of these devices portend several key practical issues. First, the strategies employed by our improved algorithms and analysis require significant mechanical and control complexity - even though they require no sensing. While we believe such mechanisms are feasible to build using the silicon MEMS technologies we advocate, it is undeniable that no such device exists yet (the M-CHIP has pixel-wise programmability, but the first generation does not have sufficient directional resolution to implement highly accurate radial strategies). For this reason, we introduce and analyze strategies composed of field sequences that we know are implementable using current (microscopic or macroscopic) technology. Each strategy is a sequence of pairs of squeezes satisfying certain "orthogonality" properties. Under these assumptions, we can ensure:

1. equilibrium stability,

2. relaxed mechanical and dynamical assumptions (the same as point 4 , above), and

3. complexity and completeness guarantees.

The framework is quite general, and applies to any set of primitive operations satisfying certain "finite equilibrium" properties (which we define) — hence it has broad applicability to a wide range of devices. In particular, we view the restricted class of fields as a vocabulary and its rules of composition as a grammar, resulting in a language of manipulation strategies. Using our grammar, the resulting strategies are guaranteed to be well-behaved.

Finally, both our radial strategies and our finite manipulation grammar have the following advantage over previous manipulation algorithms for programmable vector fields: 
previous algorithms such as those described by Böhringer and colleagues (1994a, 1996a) guarantee to uniquely orient a part, but the translational position of the part is unknown at the strategy's termination. Both of our new algorithms guarantee to position the part uniquely (up to part symmetry) in translation as well as orientation space. Like the algorithms in Böhringer's work (1994a, 1996a), the new algorithms require no sensing, and work from any initial configuration to uniquely pose the part. In particular, the initial configuration is never known to the (sensorless) execution system, which functions in an open-loop manner.

The complexity and completeness guarantees we obtain for manipulation grammars are considerably weaker than for the ideal radial strategies. For radial strategies, we show that any nonpathological planar part with finite area contact can be placed in a unique pose in $O(E)=O(n)$ steps. Under the simplified manipulation grammar, our planner is guaranteed to find a strategy if one exists (if one does not exist, the planner will signal this). However, it is not known whether there exists a strategy for every part. This lack of completeness of manipulation grammar strategies stands in contrast to the complete general squeeze and radial algorithms for which a guaranteed strategy exists for all parts. Moreover, the planning algorithm is worst-case exponential instead of merely quadratic.

Finally, the desire to implement complicated fields raises the question of control uncertainty. We close by describing how families of potential functions can be used to represent control uncertainty and analyzed for their impact on equilibria, and we give an outlook on still-open problems and future work.

\section{Experimental Apparatus: Parts Feeders}

It is often extremely costly to maintain part order throughout the manufacture cycle. For example, instead of keeping parts in pallets, they are often delivered in bags or boxes, whence they must be picked out and sorted. A parts feeder is a machine that orients such parts before they are fed to an assembly station. Currently, the design of parts feeders is a black art that is responsible for up to $30 \%$ of the cost and 50\% of workcell failures (Nevins and Whitney 1978; Boothroyd, Poli, and Murch 1982; Farnum and Davis 1986; Schroer 1987; Singer and Seering 1987). "The real problem is not part transfer but part orientation," according to Frank Riley of the Bodine Corporation (Riley 1983, p. 316, his italics). Thus, although part feeding accounts for a large portion of assembly cost, there is not much scientific basis for automating the process.

The most common type of parts feeder is the vibratory bowl feeder, where parts in a bowl are vibrated using a rotary motion, so that they climb a helical track. As they climb, a sequence of baffles and cutouts in the track create a mechanical "filter" that causes parts in all but one orientation to fall back into the bowl for another attempt at running the gauntlet (Boothroyd, Poli, and Murch 1982; Riley 1983; Sandler 1991).

Sony's APOS parts feeder (Hitakawa 1988) uses an array of nests (silhouette traps) cut into a vibrating plate. The nests and the vibratory motion are designed so that the part will remain in the nest only in one particular orientation. By tilting the plate and letting parts flow across it, the nests eventually fill up with parts in the desired orientation. Although the vibratory motion is under software control, specialized mechanical nests must be designed for each part (Moncevicz, Jakiela, and Ulrich 1991).

The reason for the success of vibratory bowl feeders and the Sony APOS system is the underlying principle of sensorless manipulation (Erdmann and Mason 1988) that allows parts positioning and orienting without sensor feedback. This principle is even more important at small scales, because sensor data will be less accurate and more difficult to obtain. The APOS system or bowl feeders are unlikely to work in the micro domain: instead, novel device designs for micromanipulation tasks are required. The theory of sensorless manipulation is the science base for developing and controlling such devices.

Reducing the amount of required sensing is an example of minimalism (Canny and Goldberg 1994; Böhringer et al. 1995b), which pursues the following agenda: for a given robot task, find the minimal configuration of resources required to solve the task. Minimalism is interesting, because doing task A without resource $\mathrm{B}$ proves that $\mathrm{B}$ is somehow inessential to the information structure of the task. In robotics, minimalism has become increasingly influential. Raibert and colleagues (1993) showed that walking and running machines could be built without static stability. Erdmann and Mason (1988) showed how to do dexterous manipulation without sensing. McGeer (1990) built a biped, kneed walker without sensors, computers, or actuators. Canny and Goldberg (1994) argued that minimalism has a long tradition in industrial manufacturing, and developed geometric algorithms for orienting parts using simple grippers and accurate, low-cost light beams. Brooks (1986) developed online algorithms that rely less extensively on planning and world models. Donald, Jennings, and Rus (1995) and Böhringer et al. (1995b) have built distributed teams of mobile robots that cooperate in manipulation without explicit communication. We intend to use these results for our experiments in micromanipulation, and to examine how they relate to our theoretical proofs of minimalist systems.

\subsection{Microfabricated Actuator Arrays}

A wide variety of micromechanical structures (devices with features in the $\mu \mathrm{m}$ range) has been built recently by using processing techniques known from the VLSI industry (see, for example, the work of Gabriel (1995), MacDonald and colleagues (1997) and MacDonald (forthcoming). Various 
microsensors and microactuators have been shown to perform successfully; e.g., a single-chip air-bag sensor is commercially available (Analog Devices 1991), and video projections using an integrated, monolithic mirror array have been demonstrated recently (Sampsell 1993) and are now starting to replace conventional projection systems. A fully integrated scanning tunneling microscope (STM) has been developed in our group (Xu, Miller, and MacDonald 1995; MacDonald et al. 1997). However, the fabrication, control, and programming of microdevices that can interact and actively change their environment remains challenging.

Problems arise from:

1. unknown material properties and the lack of adequate models for mechanisms at very small scales,

2. the limited range of motion and force that can be generated with microactuators,

3. the lack of sufficient sensor information with regard to manipulation tasks, and

4. design limitations and geometric tolerances due to the fabrication process.

Several MEMS researchers, among others (Fujita 1993; Storment et al. 1994; Liu and Will 1995; Jacobson et al. 1995; Suh et al. 1996) have proposed MEMS manipulator arrays. For an overview, see the work of Liu and Will (1995) or Böhringer and colleagues (1994a, 1994b).

Our arrays (Fig. 2) are fabricated using a SCREAM (SingleCrystal Silicon Reactive Etching and Metallization) process developed in the Cornell Nanofabrication Facility (Zhang and MacDonald 1992, Shaw, Zhang, and MacDonald 1993). The SCREAM process is low temperature, and does not interfere with traditional VLSI (Shaw and MacDonald 1996). Hence it opens the door to building monolithic microelectromechanical systems with integrated microactuators and control circuitry on the same wafer.

One of the goals of research in microactuators is to develop devices for manipulating other small components; for example, to accurately position micromachined components for inspection or assembly purposes. Fabrication constraints limit the design of most of these components (usually small chiplets made from silicon wafers) to extruded planar shapes, so manipulation in the plane is sufficient for many applications. For example, a microactuator array has been successfully employed to replace a 3-DOF stage in a scanning electron microscope (SEM) (Darling et al. 1997).

Our design is based on microfabricated torsional resonators (Mihailovich et al. 1993; Mihailovich and MacDonald 1996). Each unit device consists of a rectangular grid etched out of single-crystal silicon suspended by two rods that act as torsional springs (Fig. 3). The grid is about $200 \mu \mathrm{m}$ long, and extends $120 \mu \mathrm{m}$ on each side of the rod. The rods are 150 $\mu \mathrm{m}$ long. The current asymmetrical design has $5-\mu \mathrm{m}$ high protruding tips on one side of the grid that make contact with an object lying on top of the actuator (Fig. 4). The other side

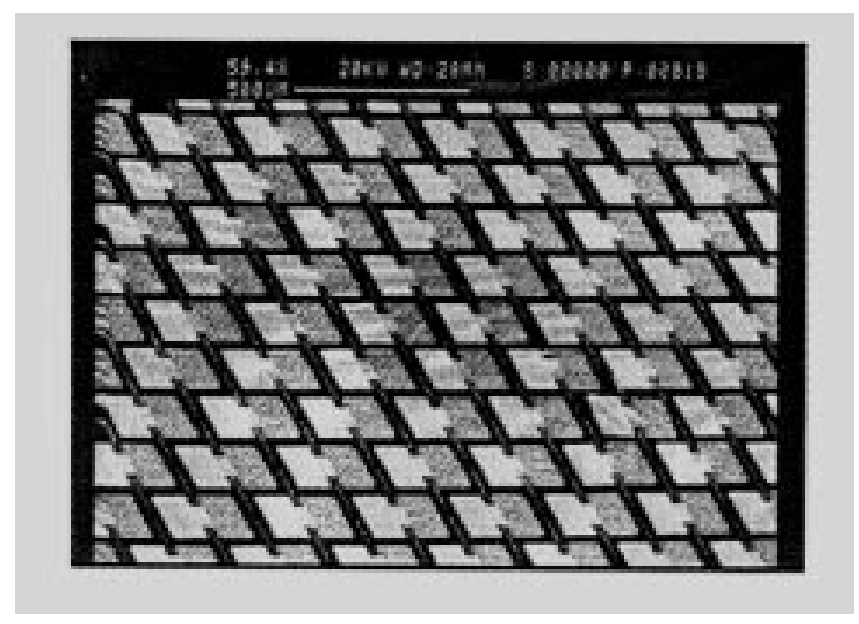

Fig. 2. A prototype M-CHIP fabricated in 1995: a large unidirectional actuator array (viewed via scanning electron microscopy). Each actuator is $180 \times 240 \mu \mathrm{m}$ in size. Detail from a $1 \mathrm{in}^{2}$ array with more than 15,000 actuators (For more pictures on device design and fabrication, see the World Wide Web at http://www.cs.cornell.edu/home/karl/ MicroActuators.)

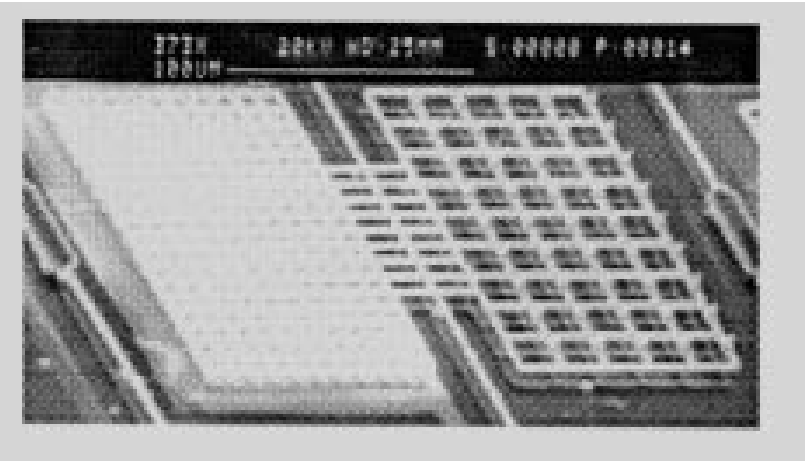

Fig. 3. Released asymmetric actuator for the M-CHIP (viewed via scanning electron microscopy): a dense grid (10 $\mu \mathrm{m}$ spacing) with an aluminum electrode underneath (left); a grid with 5- $\mu \mathrm{m}$ high poles (right).

of the actuator consists of a denser grid above an aluminum electrode. If a voltage is applied between the silicon substrate and the electrode, the dense grid above the electrode is pulled downward by the resulting electrostatic force. Simultaneously, the other side of the device (with the tips) is deflected several $\mu \mathrm{m}$ out of the plane. Hence, an object can be lifted and pushed sideways by the actuator.

Because of its low inertia (resonance in the high- $\mathrm{kHz}$ range), the device can be driven in a wide frequency range from DC to several $100 \mathrm{kHz}$ AC. Our actuators need not be operated at resonance: they can also be servoed to periodically "hit" an object on top, thereby applying both lateral and vertical forces. Our calculations, simulations, and experiments have shown that the force generated with a torsional actuator 
is approximately $10 \mu \mathrm{N}$, which corresponds to a force-perarea ratio of $100 \mu \mathrm{N} / \mathrm{mm}^{2}$, which is large enough to levitate a piece of paper $\left(1 \mu \mathrm{N} / \mathrm{mm}^{2}\right)$ or a silicon wafer $\left(10 \mu \mathrm{N} / \mathrm{mm}^{2}\right)$.

Each actuator can generate motion in one specific direction if it is activated; otherwise, it acts as a passive frictional contact. Figure 2 shows a small section of such a unidirectional actuator array, which consists of more than 15,000 individual actuators. The combination and selective activation of several actuators with different motion bias allows us to generate various motions in discrete directions, spanning the plane (Fig. 5).

The microscopic features of these actuators pose a possible disadvantage, which may make them less useful in harsh

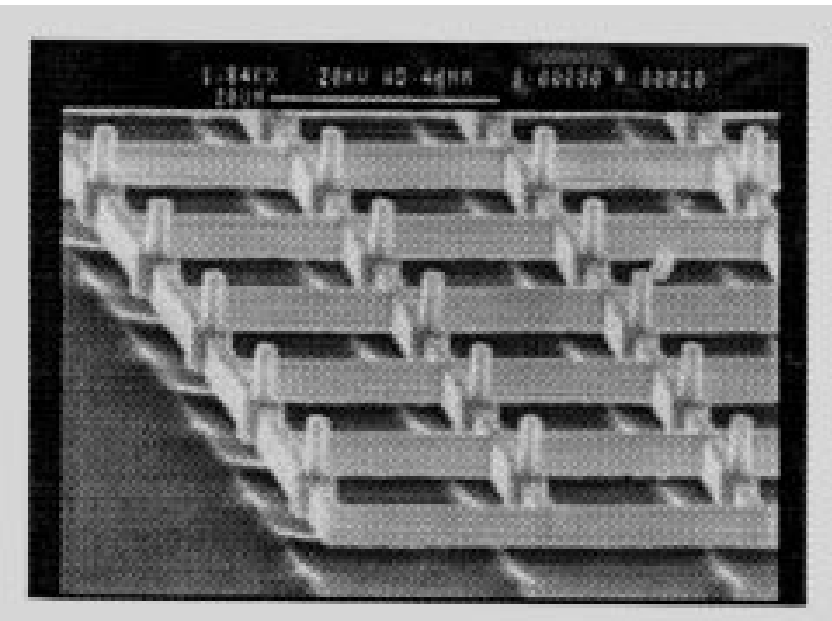

Fig. 4. Released M-CHIP actuators consisting of single-crystal silicon with 5- $\mu \mathrm{m}$ high tips.

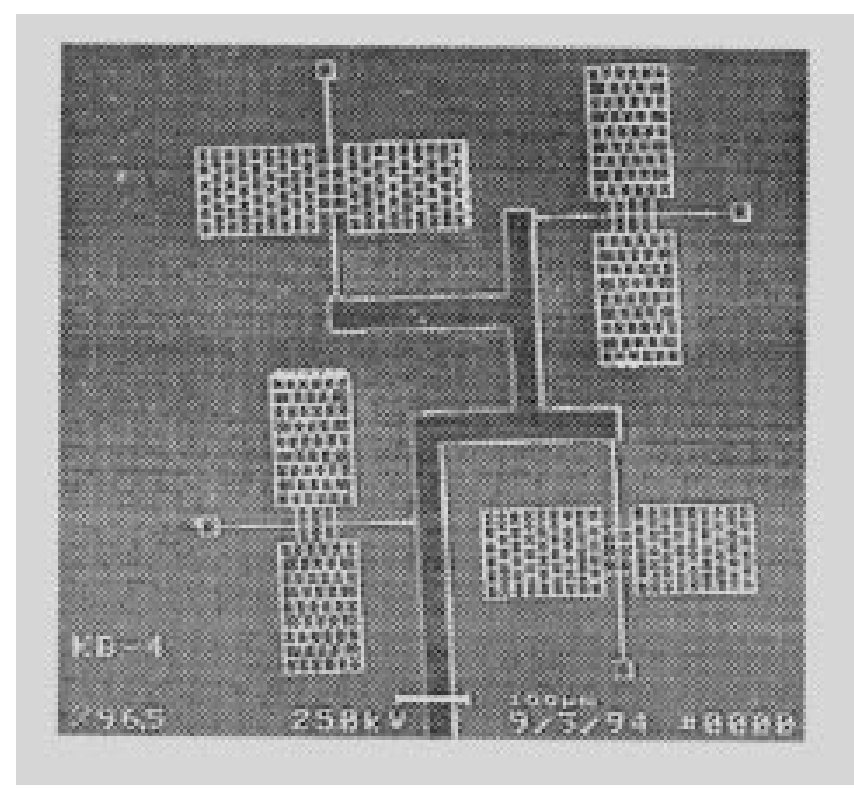

Fig. 5. Released M-CHIP prototype motion pixel, consisting of actuators oriented in four different directions. or dirty environments. Macroscopic objects and forces can easily damage microactuators. For example, careful handling is required when placing objects on the array. However, silicon is a surprisingly flexible material at microscopic scales (Peterson 1982), and extremely large elastic deformations are possible without structural damage (Taher, Saif, and MacDonald 1995). Another concern are dust particles that could jam the microactuators. As a remedy, tiny venting holes can be etched from the backside of the substrate, such that dust particles are removed by a constant flow of air. Such air jets are also useful for levitating or manipulating objects (Pister Fearing, and Howe 1990; Konishi and Fujita 1993).

The fabrication process and mechanism analysis have been described in more detail in other works (Böhringer et al. 1994a, 1994b; Böhringer, Donald, and MacDonald 1996b).

\subsection{Macroscopic Vibratory Parts Feeder}

Böhringer and colleagues (1995a) have presented a device that uses the force field created by transverse vibrations of a plate to position and align parts. The device consists of an aluminum plate that is attached to a commercially available electrodynamic vibration generator, ${ }^{1}$ with a linear travel of $0.02 \mathrm{~m}$, and the capability to produce a force of up to 500 $N$ (Fig. 6). The input signal, specifying the waveform corresponding to the desired oscillations, is fed to a single-coil armature, which moves in a constant field produced by a ceramic permanent magnet in a center-gap configuration.

For low amplitudes and frequencies, the plate moves longitudinally with no perceptible transverse vibrations. However,

\section{Model VT-100G, Vibration Test Systems, Akron, Ohio, USA.}

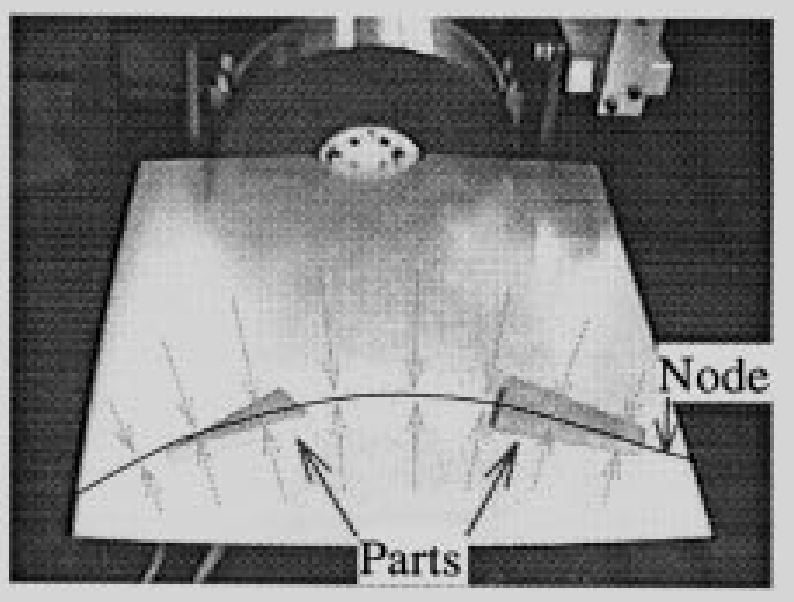

Fig. 6. Vibratory plate parts feeder: an aluminum plate (size $50 \mathrm{~cm} \times 40 \mathrm{~cm}$ ) exhibits a vibratory minimum. Parts are attracted to this nodal line, and reach equilibrium there. (See also the World Wide Web at www.ee.washington.edu/ faculty/karl/Research/VibratoryPlate.) 
as the frequency of oscillations is increased, transverse vibrations of the plate become more pronounced. The resulting motion is similar to the forced transverse vibration of a rectangular plate, clamped on one edge and free along the other three sides. This vibratory motion creates a force field in which particles are attracted to locations with minimal vibration, called the nodal lines. This field can be programmed by changing the frequency, or by employing clamps as programmable fixtures that create various vibratory nodes.

Figure 6 shows two parts, shaped like a triangle and a trapezoid, after they have reached their stable poses. To better illustrate the orienting effect, the curve showing the nodal line has been drawn by hand. Note that this device can only use the finite manipulation grammar described in Section 6.2, since it can only generate a constrained set of vibratory patterns, and cannot implement radial strategies.

\section{Equilibrium Analysis for Programmable Vector Fields}

For the generation of manipulation strategies with programmable vector fields, it is essential to be able to predict the motion of a part in the field. Particularly important is determining the stable equilibrium poses that a part can reach in which all forces and moments are balanced. This equilibrium analysis was introduced in our short conference paper (Böhringer et al. 1994a), where we presented a theory of manipulation for programmable vector fields, and an algorithm that generates manipulation strategies to orient polygonal parts without sensor feedback using a sequence of squeeze fields. We now review the algorithm from that work and give a detailed proof of its complexity bounds. The tools developed here are essential to understanding our new and improved results, and will be used throughout this paper to develop complexity bounds for our distributed manipulation algorithms.

In general, we assume that the dynamics of a part moving in the force field is governed by first-order dynamics. This assumption is based on extensive experimentation with the devices presented in Section 2. In a first-order system, the velocity of a part is directly proportional to the force acting on it. Basically, it is a rigid-body dynamical system that is heavily damped.

\subsection{Squeeze Fields and Equilibria}

In the work of Böhringer and colleagues (1994a), we proposed a family of control strategies called squeeze fields and a planning algorithm for parts orientation.

DEFINITION 1. Assume $l$ is a straight line through the origin. A squeeze field $f$ is a two-dimensional force-vector field defined as follows:

1. if $z \in \mathbb{R}^{2}$ lies on $l$, then $f(z)=0$; and
2. if $z$ does not lie on $l$, then $f(z)$ is the unit vector normal to $l$ and pointing toward $l$.

We refer to the line $l$ as the squeeze line, because $l$ lies in the center of the squeeze field. See Figure 7 for examples of squeeze fields.

Assuming quasi-static motion, an object will move perpendicularly toward the line $l$ and come to rest there. We are interested in the motion of an arbitrarily shaped (not necessarily small) part $P$. Let us call $P_{1}, P_{2}$ the regions of $P$ that lie to the left and to the right of $l$, respectively, and $c_{1}, c_{2}$ their centers of area. In a rest position, both translational and rotational forces must be in equilibrium. We obtain the following two conditions:

1. The areas $P_{1}$ and $P_{2}$ must be equal, and

2. The vector $c_{2}-c_{1}$ must be normal to $l$.

Part $P$ has a translational motion component that is normal to $l$ if condition 1 does not hold, and $P$ has a rotational motion component if condition 2 does not hold (see Fig. 8). This assumes a uniform force distribution over the surface of $P$, which is a reasonable assumption for a flat part that is in contact with a large number of elastic actuators.

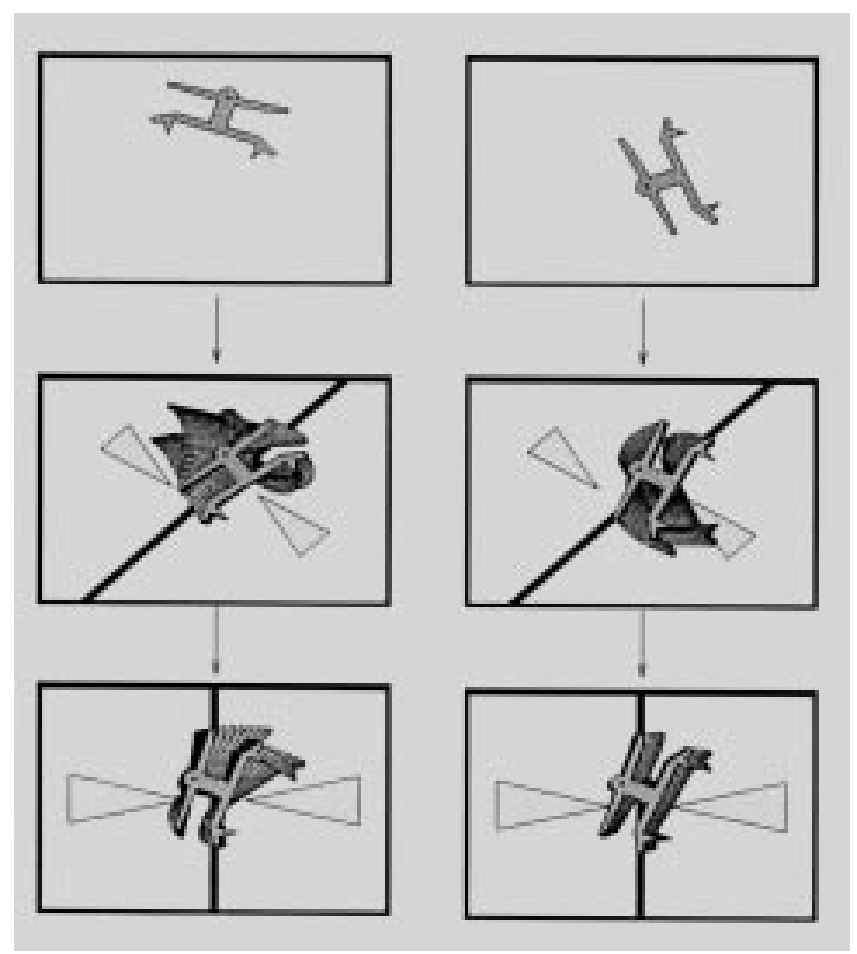

Fig. 7. Sensorless parts orienting using force-vector fields: the part reaches unique orientation after two subsequent squeezes. There exist such orientating strategies for all polygonal parts. (See the World Wide Web at www.ee.washington.edu/ faculty/karl/PFF for an animated simulation.) 


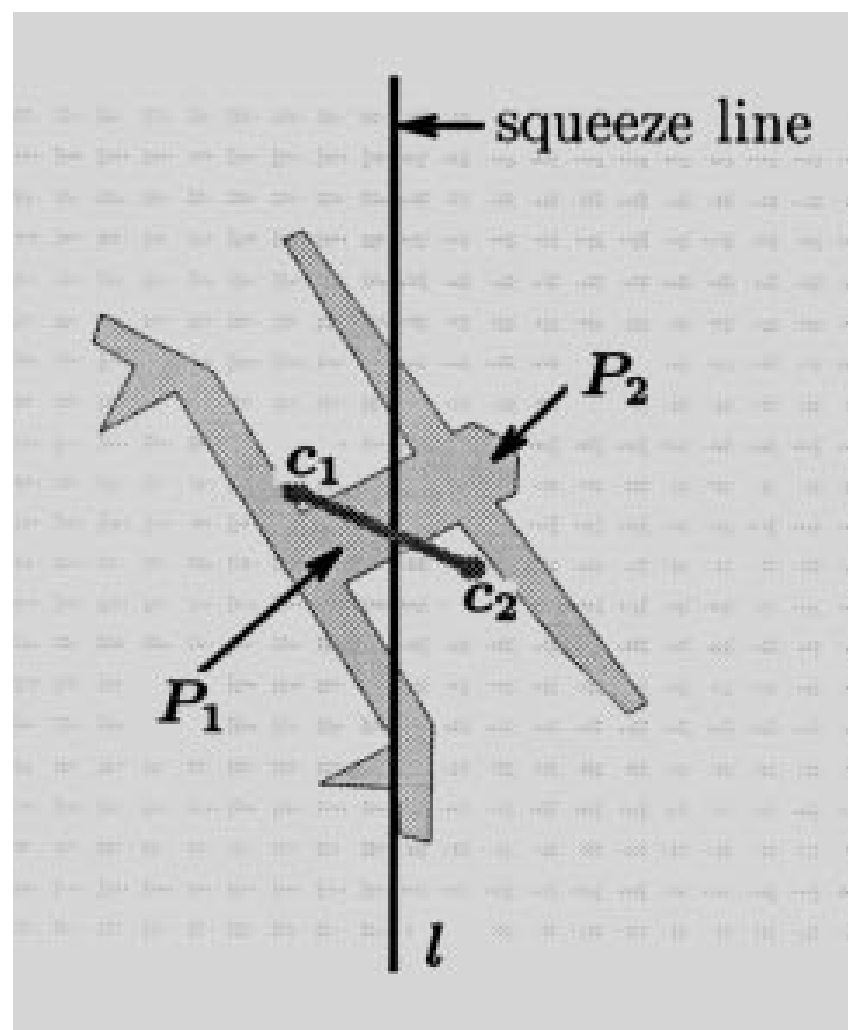

Fig. 8. Equilibrium condition: to balance the force and moment acting on $P$ in a unit squeeze field, the two areas $P_{1}$ and $P_{2}$ must be equal (i.e., $l$ must be a bisector), and the line connecting the centers of area $c_{1}$ and $c_{2}$ must be perpendicular to the node line.

DEFINITION 2. A part $P$ is in translation equilibrium if the forces acting on $P$ are balanced; $P$ is in orientation equilibrium if the moments acting on $P$ are balanced. Total equilibrium is simultaneous translation and orientation equilibrium.

Let $\left(x_{0}, y_{0}, \theta_{0}\right)$ be an equilibrium pose of $P$. $\left(x_{0}, y_{0}\right)$ is the corresponding translation equilibrium, and $\theta_{0}$ is the corresponding orientation equilibrium.

Note that conditions 1 and 2 do not imply that in equilibrium, the center of area of $P$ has to coincide with the squeeze line $l$. For example, consider a large and a small square connected by a long rod of negligible width (Fig. 9). If the rod is long enough, the center of area will lie outside of the large square. However, in equilibrium, the squeeze line $l$ will always intersect the large square.

\subsection{Polygon Bisectors and Complexity}

Consider a polygonal part $P$ in a unit squeeze field, as described in Section 3.1. In this section, we describe how to determine the orientations $\theta_{i}$ in which $P$ achieves equilibrium. This construction will show that equilibria always ex-

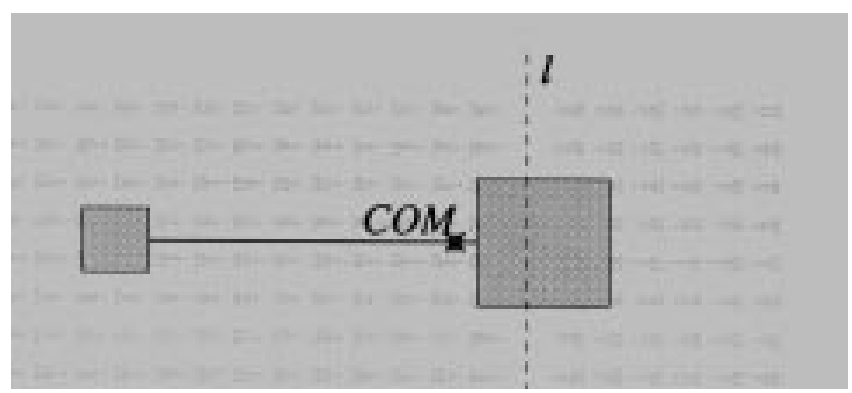

Fig. 9. A part consisting of two squares connected by a long, thin rod. The part is in total equilibrium, but its COM does not coincide with the squeeze line $l$.

$i s t$, as long as the contact areas have finite size, and that for connected parts, the orientation equilibria are discrete. More precisely, if a connected part is in equilibrium in a squeeze field, there are discrete values for its orientation and its offset from the center of the squeeze line. The equilibrium is of course independent of its position along the squeeze line. Hence, in the remainder of Section 3, when using the term "discrete equilibria," we mean that the orientation and offset of the part is discrete. We will derive upper bounds on the number of these discrete equilibria.

DEFINITION 3. A bisector of a polygon $P$ is a line that cuts $P$ into two regions of equal area.

PROPOSITION 1. Let $P$ be a polygon whose interior is connected. There exist $O\left(k n^{2}\right)$ bisectors such that $P$ is in equilibrium when placed in a squeeze field where the bisector coincides with the squeeze line. $n$ is the part complexity measured as the number of polygon vertices, and $k$ denotes the maximum number of polygon edges that a bisector can cross.

If $P$ is convex, then the number of bisectors is bounded by $O(n)$.

For most part geometries, $k$ is a small constant. ${ }^{2}$ However, in the worst case, pathological parts can reach $k=O(n)$. A spiral-shaped part (e.g., a rectilinear part) would be an example for such a pathological case, because every bisector intersects $O(n)$ polygon edges.

LEMMA 1. Given a polygon $P$ and a line $l: y=m x+c$, let $n$ be the number of vertices of $P$ :

1. there exist $O\left(n^{2}\right)$ combinatorially different ways how a line $l$ can intersect $P$;

2. let $a$ and $b$ be the intersections of bisector $l$ with the convex hull of $P$. As $m$ varies from $-\infty$ to $+\infty, a$ and $b$ progress monotonically counterclockwise about the convex hull of $P$; and

3. if the interior of $P$ is connected, then there exists a unique bisector of $P$ for every $m \in \mathbb{R}$.

2. In particular, in an earlier work (Böhringer et al. 1994a), we assumed that $k=O(1)$. 
Combinatorially equivalent intersections of polygon $P$ are all those placements of the intersecting line $l$ such that the sets of left and right polygon vertices are fixed. A necessary condition for combinatorial equivalence is that $l$ intersects the same ordered set of polygon edges.

Proof. There are $O\left(n^{2}\right)$ different placements for $l$ such that it coincides with more than one vertex of $P$. Hence, all placements of $l$ fall into one of $O\left(n^{2}\right)$ combinatorially equivalent classes. This was proven by Díaz and O'Rourke (1990, Lemma 3.1).

Assume $l$ is a bisector of $P$ with a fixed slope $m$. Since the interior of $P$ is connected, the intersection between $l$ and $P$ must be a line segment of nonzero length. Hence a translation of $l$ (e.g., toward the left) will cause a strictly monotonous decrease in the left-area segment of $P$, and vice versa. Therefore, the bisector placement of $l$ for a given slope $m$ is unique.

Consider the bisector $l$ of polygon $P$ for changing $m$ values, as described in Lemma 5. The intersections of $l$ with the convex hull of $P, a$ and $b$, progress monotonically about the convex hull. In general, this progression corresponds to a rotation and a translation of $l$.

In the following proof for Proposition 1, we investigate the relationship between the location of the bisector and the corresponding left and right areas of $P$ and its respective centers of area.

This will allow us to show that for combinatorially equivalent bisector placements, there are only a finite number of possible equilibria, and this number is bounded by $O(k)$, where $k \leq n$ is the number of polygon edges that the bisector intersects.

Proof (Proposition 1). Consider two combinatorially equivalent placements of bisector $l$ on polygon $P$. We will show that the number of equilibria for this bisector placement is bounded by $O(k)$. Since there are $O\left(n^{2}\right)$ such placements for $P$ (see Lemma 1), the total number of equilibria will be $O\left(k n^{2}\right)$.

Rotating the Bisector. Consider the line $l$ and a point $s$ that lies on $l$ (Fig. 10). The direction of $l$ is given by a vector $r$. Assume for now that the line $l$ intersects two edges of the polygon $P$ in the points $r_{1}$ and $r_{2}$. Also assume that these edges have directions $a_{1}$ and $a_{2}$. Now consider another line $l^{\prime}$ with direction $r^{\prime}$ that intersects $l$ in $s$. Assume that $l$ and $l^{\prime}$ have combinatorially equivalent intersections with polygon $P$, and that $l^{\prime}$ intersects the polygon edges in $r_{1}^{\prime}$ and $r_{2}^{\prime}$. Let us write $r_{i}=s+\rho_{i} r$ and $r_{i}^{\prime}=s+\rho_{i}^{\prime} r^{\prime}$ for $i=1,2$. Then the polygon area between $l$ and $l^{\prime}$ is

$$
A=\frac{1}{2}\left(\rho_{2}^{\prime} \rho_{2}-\rho_{1}^{\prime} \rho_{1}\right)\left(r^{\prime} \times r\right) .
$$

In the general case where $l$ and $l^{\prime}$ intersect multiple edges of some arbitrary polygon $P$ at points $r_{1}, r_{2}, \ldots, r_{k}$ and $r_{1}^{\prime}, r_{2}^{\prime}$,

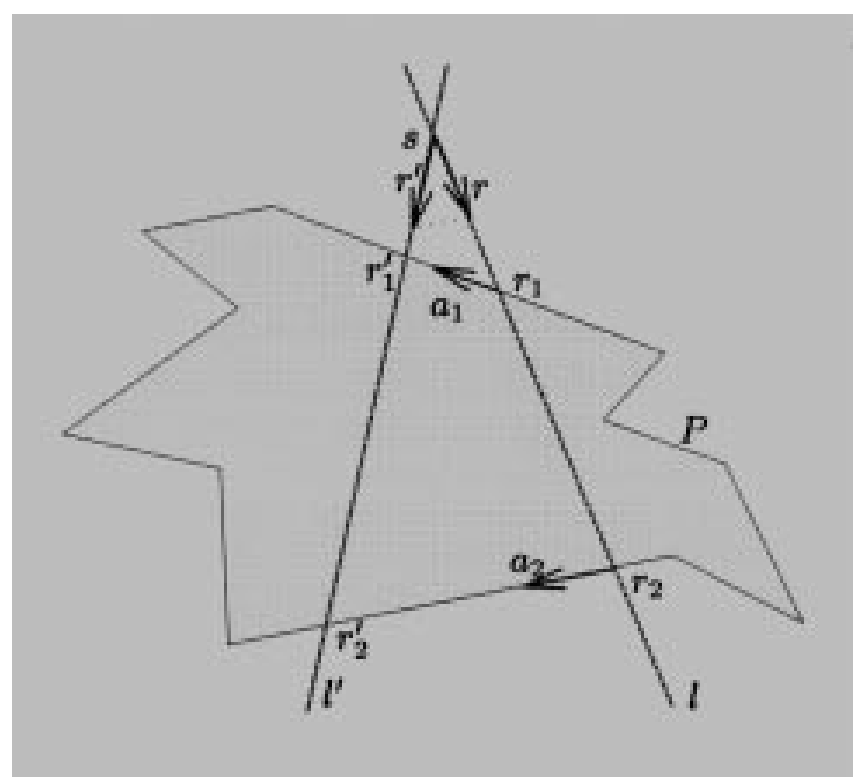

Fig. 10. Two nonparallel lines $l$ and $l^{\prime}$ in a combinatorially equivalent intersection with polygon $P$.

$\ldots, r_{k}^{\prime}\left(k\right.$ even), the polygon area between $l$ and $l^{\prime}$ is

$$
A=\frac{1}{2}\left(r^{\prime} \times r\right) \sum_{i=1}^{k}(-1)^{i} \rho_{i}^{\prime} \rho_{i} .
$$

Without loss of generality, let $\rho_{k} \neq 0$. Then $r^{\prime}$ can be written as $r^{\prime}=r+\alpha a_{k}$ for some $\alpha \in \mathbb{R}$, and the above equation becomes

$$
\begin{aligned}
A & =\frac{1}{2}\left(\left(r+\alpha a_{k}\right) \times r\right) \sum_{i=1}^{k}(-1)^{i} \rho_{i}^{\prime} \rho_{i}, \\
& =\frac{\alpha}{2}\left(a_{k} \times r\right) \sum_{i=1}^{k}(-1)^{i} \rho_{i}^{\prime} \rho_{i} .
\end{aligned}
$$

From the two vector equations $r_{i}^{\prime}=s+p_{i}^{\prime} r^{\prime}$ and $r_{i}^{\prime}=s+$ $\rho_{i} r+\lambda a_{i}, \lambda \in \mathbb{R}$, we can determine $\rho_{i}^{\prime}$ as

$$
\rho_{i}^{\prime}=\frac{\rho_{i}\left(a_{i} \times r\right)}{\left(a_{i} \times r\right)+\alpha\left(a_{i} \times a_{k}\right)} .
$$

If we also choose the edge-direction vectors $a_{i}$ such that $\left(a_{i} \times\right.$ $r)=1$, then eqs. (1) and (2) simplify to the following rational functions in $\alpha$ :

$$
\begin{aligned}
\rho_{i}^{\prime} & =\frac{\rho_{i}}{1+\alpha\left(a_{i} \times a_{k}\right)}, \\
A & =\frac{\alpha}{2} \sum_{i=1}^{k}(-1)^{i} \frac{\rho_{i}^{2}}{1+\alpha\left(a_{i} \times a_{k}\right)} .
\end{aligned}
$$

Let us look at the denominator $d_{i}(\alpha)=1+\alpha\left(a_{i} \times a_{k}\right)$ in more detail. This is important because we shall see that in 
all formulas we will obtain, the denominators consist only of $d_{i}(\alpha)$. For an arbitrary polygon, $d_{i}$ is a linear function of $\alpha$. If all $a_{i}$ are parallel, then $d_{i}=1$. If the polygon is rectilinear, i.e., all $a_{i}$ are either parallel or perpendicular, then $d_{i}(\alpha)=1$ if $a_{i} \| a_{k}$, and $d_{i}(\alpha)=1+\alpha a_{\perp}$ if $a_{i} \perp a_{k}$, where $a_{\perp}$ is constant. So in this case, there are only two different constant denominators, one of which is 1 .

Translating the Bisector. We now consider the case where $l^{\prime}$ shifts parallel (Fig. 11). Analogously to the previous paragraph, let $r_{i}^{\prime}=s^{\prime}+\rho_{i}^{\prime} r^{\prime}$, and $r_{i}^{\prime \prime}=s^{\prime \prime}+\rho_{i}^{\prime \prime} r^{\prime}$. Also, let the vector between $s^{\prime}$ and $s^{\prime \prime}$ be $s^{\prime \prime}-s^{\prime}=\beta a_{2}$. Then the polygon area between $l^{\prime}$ and $l^{\prime \prime}$ is

$$
\begin{aligned}
B & =\beta a_{2} \times \frac{1}{2}\left(\left(r_{2}^{\prime}+r_{2}^{\prime \prime}\right)-\left(r_{1}^{\prime}+r_{1}^{\prime \prime}\right)\right) \\
& =\frac{\beta}{2}\left(\rho_{2}^{\prime}+\rho_{2}^{\prime \prime}-\rho_{1}^{\prime}-\rho_{1}^{\prime \prime}\right)\left(a_{2} \times\left(r+\alpha a_{2}\right)\right), \\
& =\frac{\beta}{2}\left(\rho_{2}^{\prime}+\rho_{2}^{\prime \prime}-\rho_{1}^{\prime}-\rho_{1}^{\prime \prime}\right)
\end{aligned}
$$

In the general case, $l^{\prime}$ and $l^{\prime \prime}$ intersect multiple edges of some arbitrary polygon $P$ at points $r_{1}^{\prime}, r_{2}^{\prime}, \ldots, r_{k}^{\prime}$ and $r_{1}^{\prime \prime}, r_{2}^{\prime \prime}, \ldots, r_{k}^{\prime \prime}$. Now the $\rho_{i}^{\prime \prime}$ can be determined from the two vector equations $r_{i}^{\prime \prime}=r_{i}^{\prime}+\lambda a_{i}, \lambda \in \mathbb{R}$, and $r_{i}^{\prime \prime}=s^{\prime \prime}+\rho_{i}^{\prime \prime} r^{\prime}$ :

$$
\begin{aligned}
\rho_{i}^{\prime \prime} & =\rho_{i}^{\prime}-\beta \frac{a_{i} \times a_{k}}{a_{i} \times r^{\prime}}, \\
& =\rho_{i}^{\prime}-\beta \frac{a_{i} \times a_{k}}{1+\alpha\left(a_{i} \times a_{k}\right)}, \\
& =\frac{\rho_{i}-\beta\left(a_{i} \times a_{k}\right)}{1+\alpha\left(a_{i} \times a_{k}\right.} .
\end{aligned}
$$

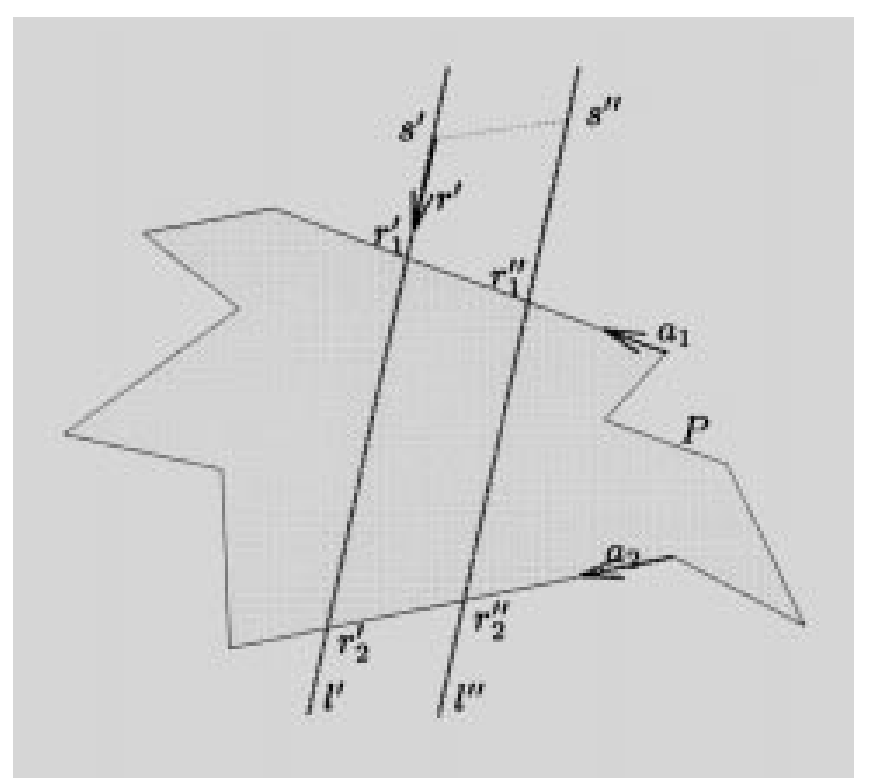

Fig. 11. Two parallel lines $l^{\prime}$ and $l^{\prime \prime}$ in combinatorially equivalent intersection with polygon $P$.
Then the polygon area between $l^{\prime}$ and $l^{\prime \prime}$ is

$$
\begin{aligned}
B & =\frac{\beta}{2} \sum_{i=1}^{k}(-1)^{i}\left(\rho_{i}^{\prime}+\rho_{i}^{\prime \prime}\right), \\
& =\frac{\beta}{2} \sum_{i=1}^{k}(-1)^{i} \frac{\left(\rho_{i}-\beta\left(a_{i} \times a_{k}\right)\right.}{1+\alpha\left(a_{i} \times a_{k}\right)} .
\end{aligned}
$$

This is a quadratic polynomial in $\beta$ (unless all $a_{i}$ are parallel, in which case it simplifies to the linear equation $B=$ $\left.\beta \sum_{i=1}^{k}(-1)^{i} \rho_{i}\right)$.

Maintaining the Bisector Property. From the above two paragraphs, we see that if the bisector $l$ is rotated to $l^{\prime}$, then the left and right areas are changed by a value $A(\neq 0$ in general) as described in eq. (4). Hence, a subsequent shift of $l^{\prime}$ is necessary to restore the bisector property, by changing the areas by a value $B$, as described in eq. (7).

This implies the condition $A+B=0$, with $A$ and $B$ given by eqs. (4) and (7):

$$
\begin{aligned}
A+B & =\frac{1}{2} \sum_{i=1}^{k}(-1)^{i} \frac{\alpha \rho_{i}^{2}+2 \beta \rho_{i}-\beta^{2}\left(a_{i} \times a_{k}\right)}{1+\alpha\left(a_{i} \times a_{k}\right)} . \\
& =0
\end{aligned}
$$

This equation ensures that $l$ is a bisector of $P$. It is a necessary and sufficient condition for translation equilibrium in a unitsqueeze field. Equation (8) is a rational equation in $\alpha$, and a quadratic polynomial equation in $\beta$. Hence for all combinatorially equivalent bisectors, we can obtain an explicit formula to describe $\beta$ as a function of $\alpha$.

In general, eq. (8) is equivalent to a polynomial in $\alpha$ and $\beta$ whose degree depends on the number $k$ of polygon edges intersected by the bisectors $l, l^{\prime}$, or $l^{\prime \prime}$. The degree of this polynomial is limited by $k$ for $\alpha$, and by 2 for $\beta$. In the rectilinear case, the degrees for $\alpha$ and $\beta$ are limited by 2 . In the case where all $a_{i}$ are parallel, eq. (8) simplifies to a linear equation: $\sum_{i=1}^{k}(-1)^{i}\left(\alpha \frac{\rho_{i}}{2}+\beta\right) \rho_{i}=0$.

Moment Equilibrium. After rotating (parameter $\alpha$, obtain $l^{\prime}$ ) and translating (parameter $\beta$, obtain $l^{\prime \prime}$ ) the bisector $l$, its intersections with the polygon edges move from $r_{i}$ to

$$
\begin{aligned}
r_{i}^{\prime \prime} & =s+\rho_{i}^{\prime \prime} r^{\prime}+\beta a_{k}, \\
& =s+\frac{\rho_{i}-\beta\left(a_{i} \times a_{k}\right)}{1+\alpha\left(a_{i} \times a_{k}\right)}\left(r+\alpha a_{k}\right)+\beta a_{k} .
\end{aligned}
$$

If all $a_{i}$ are parallel, this simplifies to $r_{i}^{\prime \prime}=s+\rho_{i} r+\left(\alpha \rho_{i}+\right.$ $\beta) a_{k}$.

Suppose that $c_{l}$ and $c_{r}$, are the left and the right centers of area of $P$, and $A_{l}$ and $A_{r}$ are the respective area sections, so $A_{l}+A_{r}=A$. We are interested in how these points change when the bisector changes. Note that always $c=$ $\frac{1}{A}\left(A_{l} c_{l}+A_{r} c_{r}\right)$, and if $P$ is bisected (i.e., $\left.A_{l}=A_{r}=\frac{1}{2} A\right)$ then $c=\frac{1}{2}\left(c_{l}+c_{r}\right)$. 
We consider the area between $l$ and $l^{\prime \prime}$, which can be written as a sum of quadrangles $\left(r_{i}, r_{k}, r_{k}^{\prime \prime}, r_{i}^{\prime \prime}\right)$. The weighted center area of this area can be determined as

$$
\begin{aligned}
C & =\sum_{i=1}^{k}(-1)^{i} \frac{1}{6}\left(\left(r_{i}+r_{k}\right)\left(r_{i} \times r_{k}\right)+\left(r_{k}+r_{k}^{\prime \prime}\right)\left(r_{k} \times r_{k}^{\prime \prime}\right)\right. \\
& \left.+\left(r_{k}^{\prime \prime}+r_{i}^{\prime \prime}\right)\left(r_{k}^{\prime \prime} \times r_{i}^{\prime \prime}\right)+\left(r_{i}^{\prime \prime}+r_{i}\right)\left(r_{i}^{\prime \prime} \times r_{i}\right)\right)
\end{aligned}
$$

For the left areas, the following relationship holds (assuming $\left.A_{l}^{\prime \prime} \neq 0\right)$ :

$$
\begin{aligned}
A_{l}^{\prime \prime} c_{l}^{\prime \prime} & =A_{l} c_{l}+C \\
\Rightarrow \quad c_{l}^{\prime \prime} & =\frac{A_{l}}{A_{l}^{\prime \prime}} c_{l}+\frac{1}{A_{l}^{\prime \prime}} C,
\end{aligned}
$$

and similarly, for the right areas (assuming $A_{r}^{\prime \prime} \neq 0$ ):

$$
c_{r}^{\prime \prime}=\frac{A_{r}}{A_{r}^{\prime \prime}} c_{r}-\frac{1}{A_{l}^{\prime \prime}} C .
$$

Hence,

$$
c_{r}^{\prime \prime}-c_{r}^{\prime \prime}=\frac{A_{l}}{A_{l}^{\prime \prime}} c_{t}-\frac{A_{r}}{A_{r}^{\prime \prime}} c_{r}+\left(\frac{1}{A_{l}^{\prime \prime}}+\frac{1}{A_{r}^{\prime \prime}}\right) C .
$$

Both $l$ and $l^{\prime \prime}$ are bisectors, so $A_{l}=A_{r}=A_{l}^{\prime \prime}=A_{r}^{\prime \prime}=\frac{A}{2}$, and

$$
c_{l}^{\prime \prime}-c_{r}^{\prime \prime}=c_{l}-c_{r}+\frac{4}{A} C .
$$

For orientation equilibrium, we require that the line connecting the centers of area, $c_{r}^{\prime \prime}-c_{l}^{\prime \prime}$, and the direction of the bisector $r^{\prime}$, are perpendicular:

$$
\begin{aligned}
\left(c_{l}^{\prime \prime}-c_{r}^{\prime \prime}\right) \cdot r^{\prime} & =\left(c_{l}-c_{r}+\frac{4}{A} C\right) \cdot r^{\prime}, \\
& =0 .
\end{aligned}
$$

The value of $C=C(\alpha, \beta)$ can be determined by using eqs. (9) and (10), and the equation $r^{\prime}=r+\alpha a_{k}$. Equation (11) is a necessary and sufficient condition for orientation equilibrium.

By using the expressions derived in eqs. (1)-(10), both eqs. (8) (for translation equilibrium) and (11) (for orientation equilibrium) can be expressed with rational functions in $\alpha$ and $\beta$ whose numerator (respectively, denominator) degrees are $O(k)$ (respectively, $O(1)$ ) for $\alpha$ and 2 for $\beta$. Hence, we can obtain a system of two polynomial equations of degree $O(k)$ for $\alpha$ and 2 for $\beta$. This system has at most $O(k)$ solutions, resulting in $O(k)$ total equilibria for bisector placements that are combinatorially equivalent. Since there are $\left(n^{2}\right)$ combinatorially different bisector placements, there are at most $O\left(k n^{2}\right)$ total equilibria. []

\subsection{Planning of Manipulation Strategies}

In this section, we present an algorithm for sensorless parts alignment with squeeze fields (Böhringer et al., 1994a; Böhringer, Donald, and MacDonald 1996a). Recall from Section 3.2 that in squeeze fields, the equilibria for connected polygons are discrete (modulo a neutrally stable translation parallel to the squeeze line, which we will disregard for the remainder of Section 3).

To model our actuator arrays and vibratory devices, we made the following assumptions:

Density: the generated forces can be described by a vector field, i.e., the individual microactuators are dense compared to the size of the moving part; and

2Phase: the motion of a part has two phases: (1) pure translation towards $l$ until the part is in translation equilibrium, and (2) motion in translation equilibrium until orientation equilibrium is reached.

Note that due to the elasticity and oscillation of the actuator surfaces, we can assume continuous area contact, and not just contact in three or a few points. If a part moves while in translation equilibrium, in general the motion is not a pure rotation, but also has a translational component. Therefore, relaxing the 2 Phase assumption is one of the key results of this paper.

DEFINITION 4. Let $\theta$ be the orientation of a connected polygon $P$ in a squeeze field, and let us assume that condition 1 holds. The turn function $t: \theta \rightarrow\{-1,0,1\}$ describes the instantaneous rotational motion of $P$ :

$$
t(\theta)=\left\{\begin{array}{cl}
1 & \text { if } P \text { will turn counterclockwise, } \\
-1 & \text { if } P \text { will turn clockwise, } \\
0 & \text { if } P \text { is in total equilibrium. }
\end{array}\right.
$$

See Figure 12 for an illustration. The turn function $t(\theta)$ can be obtained, for example, by taking the sign of the lifted moment $M_{P}(\mathbf{z})$ for poses $\mathbf{z}=(x, y, \theta)$, in which the lifted force $f_{P}(\mathbf{z})$ is zero.

Definition 6 immediately implies the following lemma.

LEMMA 2 (Böhringer, MacDonald, and Donald 1996a). Let $P$ be a polygon with orientation $\theta$ in a squeeze field such that condition 1 holds. $P$ is stable if $t(\theta)=0, t(\theta+) \leq 0$, and $t(\theta-) \geq 0$; otherwise, $P$ is unstable.

Proof. Assume the part $P$ is in a pose $(x, y, \theta)$ such that condition 1 is satisfied. This implies that the translational forces acting on $P$ balance out. If in addition $t(\theta)=0$, then the effective moment is zero, and $P$ is in total equilibrium. Now consider a small perturbation $\delta_{\theta}>0$ of the orientation $\theta$ of $P$ while condition 1 is still satisfied. For a stable equilibrium, the moment resulting from the perturbation $\delta_{\theta}$ must not aggravate, but rather counteract, the perturbation. This is true if and only if $t\left(\theta+\delta_{\theta}\right) \leq 0$ and $t\left(\theta-\delta_{\theta}\right)>0$. 


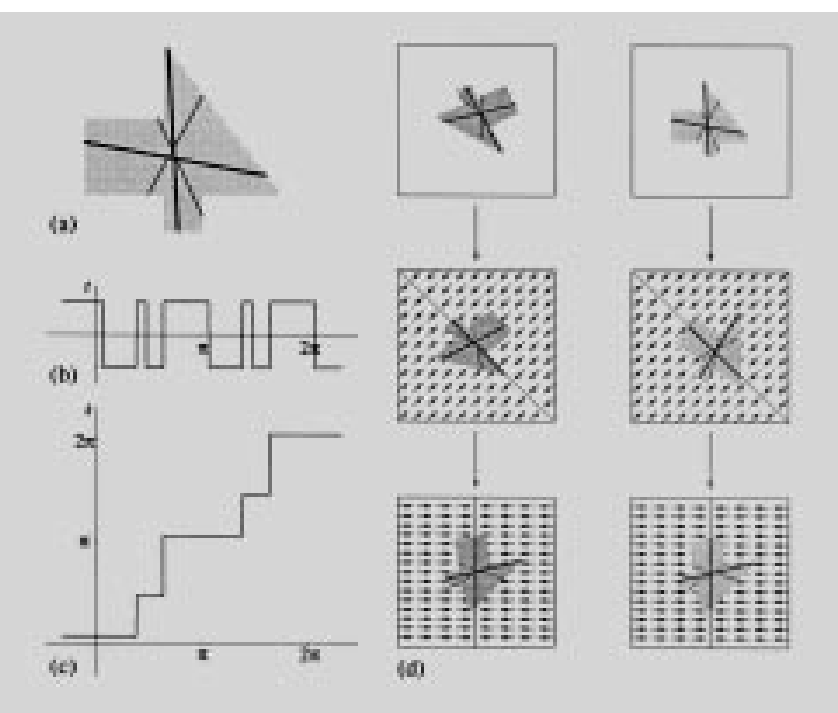

Fig. 12. (a) Polygonal part: stable (thick line) and unstable (thin line) bisectors are also shown. (b) Turn function, which predicts the orientations of the bisectors. Stable (respectively, unstable) bisectors correspond to angles at which the turn function changes from +1 to -1 (respectively, from -1 to +1 ). (c) Squeeze function, constructed from the turn function. (d) Alignment strategy for two arbitrary initial configurations. (See the World Wide Web at www.ee.washington.edu/faculty/karl/Research/ for an animated simulation.)

Using this lemma, we can identify all stable orientations, which allows us to construct the squeeze function (Goldberg 1993) of $P$ (see Fig. 12c); i.e., the mapping from an initial orientation of $P$ to the stable equilibrium that it will reach in the squeeze field:

LEMMA 3. Let $P$ be a polygonal part on an actuator array $\mathcal{A}$ such that Density and 2Phase hold. Given the turn function $t$ of $P$, its corresponding squeeze function $s: \mathbb{S}^{1} \rightarrow \mathbb{S}^{1}$ is constructed as follows:

1. all stable equilibrium orientations $\theta$ map identically to $\theta$;

2. all unstable equilibrium orientations map (by convention $^{3}$ ) to the nearest counterclockwise stable orientation; and

3. all orientations $\theta$ with $t(\theta)=1(-1)$ map to the nearest counterclockwise (clockwise) stable orientation.

Then, $s$ describes the orientation transition of $P$ induced by $\mathcal{A}$.

Proof. Assume that part $P$ initially is in pose $(x, y, \theta)$ in array $\mathcal{A}$. Because of the 2 Phase assumption, we can assume that $P$ translates toward the center line $l$ until condition 1 is

3. Equally, one could define $t$ to map unstable equilibrium orientations to the nearest clockwise stable equilibrium. This choice for a set of measure zero does not affect our subsequent analysis and algorithms. satisfied without changing its orientation $\theta . P$ will change its orientation until the moment is zero, i.e., $t=0$ : a positive moment, $(t>0)$ causes counterclockwise motion, and a negative moment $(t<0)$ causes clockwise motion until the next root of $t$ is reached.

We conclude that any connected polygonal part, when put in a squeeze field, reaches one of a finite number of possible orientation equilibria (Böhringer et al. 1994a; Böhringer, Donald, and MacDonald 1996a). The motion of the part and, in particular, the mapping between initial orientation and equilibrium orientation is described by the squeeze function, which is derived from the turn function (as described in Lemma 3). Note that all squeeze functions derived from turn functions are monotone step-shaped functions.

Goldberg (1993) has given an algorithm that automatically synthesizes a manipulation strategy to uniquely orient a part, given its squeeze function. While Goldberg's algorithm was designed for squeezes with a robotic parallel-jaw gripper, in fact, it is more general, and can be used for arbitrary monotone step-shaped squeeze functions. The output of Goldberg's algorithm is a sequence of angles that specify the required directions of the squeezes; therefore, these angles specify the direction of the squeeze line in our force-vector fields (for example, the two-step strategies in Figures 7 and 12d). It is important to note that the equilibria obtained by a MEMS squeeze field and by a parallel-jaw gripper will typically be different, even when the squeeze directions are identical. For example, to see this, consider squeezing a square-shaped part (Fig. 13). Stable and unstable equilibria are reversed. This shows that our mechanical analysis of equilibrium is different from that of the parallel-jaw gripper. Let us summarize these results in the following statements.

PROPOSITION 2. Let $P$ be a polygon whose interior is connected. There exists an alignment strategy consisting of a

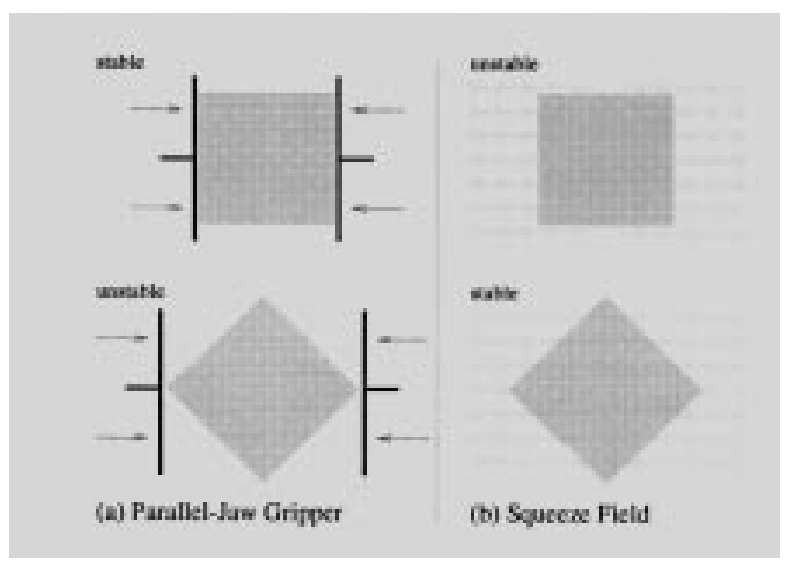

Fig. 13. Equilibrium configurations for a square-shaped part using (a) a frictionless parallel-jaw gripper, and (b) a MEMS squeeze field. In this example, stable and unstable equilibria are reversed. 
sequence of squeeze fields that uniquely orients $P$ up to symmetries.

Since the strategies of Proposition 2 consist of fields with squeeze lines at arbitrary angles through the origin, we call them general $\mathbb{S}^{1}$ squeeze strategies, or, henceforth, general squeeze strategies.

COROLlARY 1. The alignment strategies of Proposition 2 have $O\left(k n^{2}\right)$ steps, and they may be computed in time $O\left(k^{2} n^{4}\right)$, where $k$ is the maximum number of edges that a bisector of $P$ can cross. In the case where $P$ is convex, the alignment strategy has $O(n)$ steps, and can be computed in time $O\left(n^{2}\right)$.

Proof. Proposition 1 states that a polygon with $n$ vertices has $E=O\left(k n^{2}\right)$ stable orientation equilibria in a squeeze field $(O(n)$ if $P$ is convex). This means that the image of its corresponding squeeze function is a set of $E$ discrete values. Given such a squeeze function, Goldberg's algorithm (Goldberg 1993) constructs alignment strategies with $O(E)$ steps. Planning complexity is $O\left(E^{2}\right)$.

The strategies of Goldberg (1993) have the same complexity bounds for convex and nonconvex parts, because when using squeeze grasps with a parallel-jaw gripper, only the convex hull of the part need be considered. This is not the case for programmable vector fields, where manipulation strategies for nonconvex parts are more expensive. As described in Section 3.2, there could exist parts that have $E=\Omega\left(k n^{2}\right)$ orientation equilibria in a squeeze field, which would imply alignment strategies of length $\Omega\left(k n^{2}\right)$ and planning complexity $\Omega\left(k^{2} n^{4}\right)$.

Note that the turn and squeeze functions have a period of $\pi$, due to the symmetry of the squeeze field; rotating the field by an angle of $\pi$ produces an identical vector field. Rotational symmetry in the part also introduces periodicity into these functions. Hence, general squeeze strategies (see Proposition 2) orient a part up to symmetry, that is, up to symmetry in the part and in the squeeze field. Similarly, the grasp plans based on squeeze functions in the work of Goldberg (1993) can orient a part with a macroscopic gripper only modulo symmetry in the part and in the gripper. ${ }^{4}$ Since in Goldberg's (1993) work we reduce to the squeeze-function algorithm, it is not surprising that this phenomenon is also manifested for squeeze-vector fields as well. A detailed discussion of parts orientation modulo symmetry has been provided (Goldberg 1993).

The algorithm in this section uniquely orients a part (up to symmetry); however, its position cannot be predicted precisely. In Section 6, we will present new and improved manipulation algorithms that position and orient parts uniquely, and also reduce the number of equilibria to $E=O(k n)$. In Section 6.2 we will show that the algorithm described in this

4. Parallel-jaw gripper symmetry is also modulo $\pi$. section can be extended easily such that unique positioning and orienting can be achieved.

Squeeze fields may be generalized to the case where $l$ is slightly curved, as in the "node" of the vibrating plate in Figure 6 (details are available (Böhringer 1995a)). The remaining sections of this paper investigate using more exotic fields (not simple squeeze patterns) to:

- allow disconnected polygons,

- relax the 2 Phase assumption,

- reduce the planning complexity,

- reduce the number of equilibria,

- reduce the execution complexity (strategy length), and

- determine feasibility results and limitations for manipulation with general force fields.

\subsection{Relaxing the 2Phase Assumption}

In Section 3.3, the 2Phase assumption allowed us to determine successive equilibrium positions in a sequence of squeezes, by a quasi-static analysis that decouples translational and rotational motion of the moving part. For any part, this obtains a unique orientation equilibrium (after several steps). If the 2Phase assumption is relaxed, we obtain a dynamic manipulation problem, in which we must determine the equilibria $(x, \theta)$ given by the part orientation $\theta$ and the offset $x$ of its center of mass from the squeeze line. A stable equilibrium is a $\left(x_{i}, \theta_{i}\right)$ pair in $\mathbb{R} \times \mathbb{S}^{1}$ that acts as an attractor (the $x$ offset in an equilibrium is, surprisingly, usually not 0 ; see Fig. 9). Again, we can compute these $\left(x_{i}, \theta_{i}\right)$ equilibrium pairs exactly, as outlined in Section 3.2.

Considering $\left(x_{i}, \theta_{i}\right)$ equilibrium pairs has another advantage. We can show that, even without the 2 Phase assumption, after two successive, orthogonal squeezes, the set of stable poses of any part can be reduced from $\mathcal{C}=\mathbb{R}^{2} \times \mathbb{S}^{1}$ to a finite subset of $\mathcal{C}$ (the configuration space of part $P$ ); see claim 1 (Section 6.2). Subsequent squeezes will preserve the finiteness of the state space. This will significantly reduce the complexity of a task-level motion planner. Hence, if assumption 2 Phase is relaxed, this idea still enables us to simplify the general motion-planning problem (as formulated, for example, by Lozano-Pérez, Mason, and Taylor (1984)) to that of Erdmann and Mason (1988). Conversely, relaxing assumption 2Phase raises the complexity from the "linear" planning scheme of Goldberg (1993) to the forward-chaining searches of Erdmann and Mason (1988) or Donald (1990).

\section{Lower Bounds: What Programmable Vector Fields Cannot Do}

We now present "lower bounds"—constituting vector fields and parts with pathological behavior, making them unusable for positioning. These counterexamples show that we must be careful in choosing programmable vector fields, and that, a priori, it is not obvious when a field is well behaved. 


\subsection{Unstable Fields}

In Section 3, we saw that in a vector field with a simple squeeze pattern (see again Fig. 7), polygonal parts reach certain equilibrium poses. This raises the question of a general classification of all those vector fields in which every part has stable equilibria. There exist vector fields that do not have this property, even though they are very similar to a simple squeeze.

DEFINITION 5. A skewed field $f_{S}$ is a vector field given by $f_{S}(x, y)=-\operatorname{sign}(x)(1, \epsilon)$, where $0 \neq \epsilon \in \mathbb{R}$.

Proposition 3. A skewed field induces no stable equilibrium on a disk-shaped part.

Proof. Consider Figure 14, which shows a skewed field with $\epsilon=-\frac{2}{3}$ : only when the center of the disk coincides with the center of the squeeze pattern do the translational forces acting on the disk balance. But it will still experience a positive moment that will cause rotation.

Proposition 4. A diagonally skewed field $(\epsilon= \pm 1)$ induces no stable equilibrium on a square-shaped part.

Proof. To reach equilibrium in a diagonally skewed field, the squeeze line has to bisect the part such that the connector between the left and the right centers of area is diagonal (i.e., parallel to the force vectors). An analysis similar to the proof of Proposition 1 (Section 3.2) shows that for a square, no bisector placement can achieve an angle with the connector of less than $83^{\circ}$.

Propositions 3 and 4 show that skewed squeeze fields cannot be used for open-loop positioning or orienting of parts, since there may not exist any stable equilibria. However, skewed squeeze fields may be very useful if our goal is to continuously rotate a part (e.g., for inspection purposes).

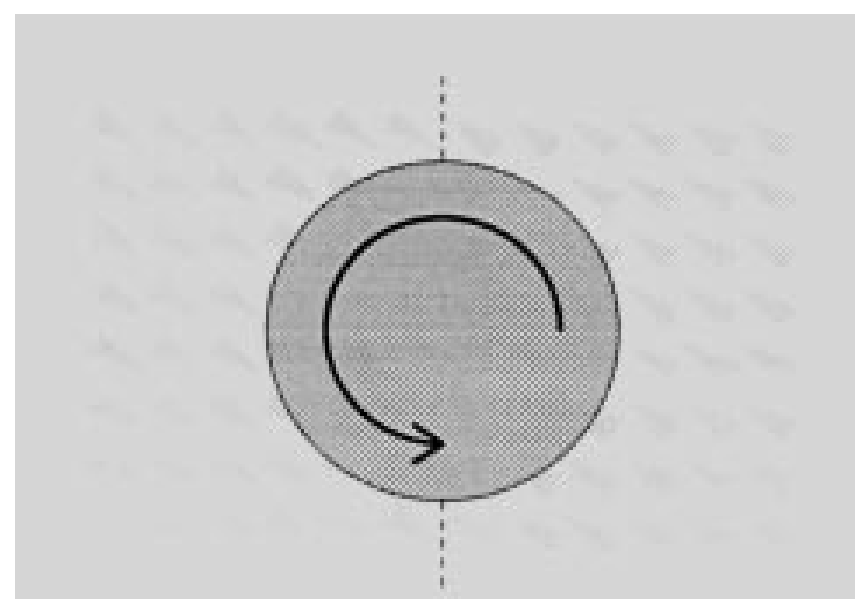

Fig. 14. Unstable part in the skewed squeeze field $\left(\epsilon=-\frac{2}{3}\right)$. The disk with its center on the squeeze line will keep rotating; moreover, it has no stable equilibrium in this field.

\subsection{Unstable Parts}

Similarly, we would like to identify the class of all those parts that always reach stable equilibria in particular vector fields. From Section 3, we know that connected polygons in simple squeeze fields satisfy this condition. This property relies on finite area contacts: it does not hold for point contacts. As a counterexample, consider the part $P_{S}$ in Figure 15.

Proposition 5. There exist parts that do not have stable equilibria in a simple squeeze field.

Proof. The S-shaped part in Figure 15 has four rigidly connected "feet" with small contact surfaces. As the area of each of these four feet approaches zero, the part has no stable equilibrium in a simple squeeze field. There is only one orientation for the part in which both force and moment balance out, and this orientation is unstable.

In Section 5.2, we discuss this phenomenon in greater detail, after the tools necessary for a complete mathematical analysis have been developed.

Finally, the number of stable equilibria of a given part influences both the planning complexity and the plan length of an alignment strategy. It also affects the resolution of the vector field that is necessary to perform a strategy accurately. Even though all parts we have considered exhibit only one or two orientation equilibria, there exist no tight bounds on the maximum number of orientation equilibria in a unit squeeze field.

PROPOSITION 6. Let $n$ be the number of vertices of a polygon $P$, and let $k$ be the maximum number of edges that a bisector of $P$ can cross:

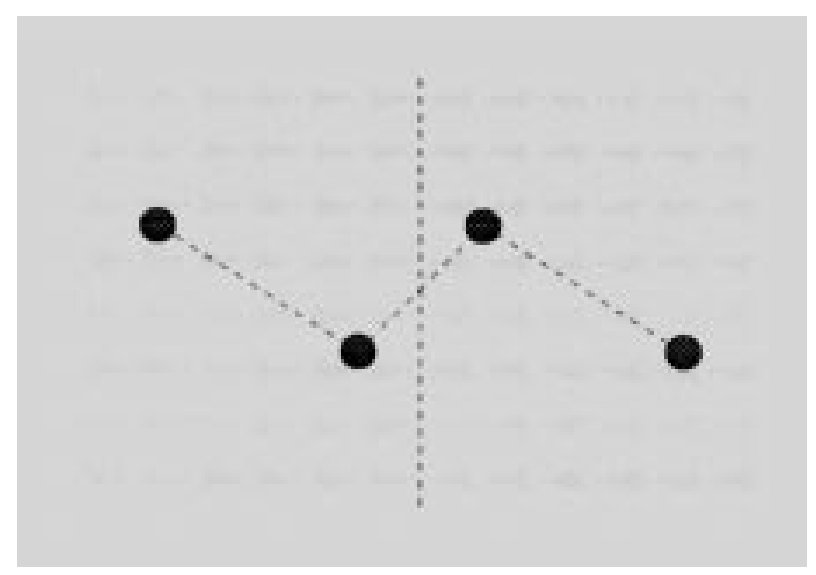

Fig. 15. The S-shaped part $P_{S}$ with four rigidly connected point-contact "feet" in unstable total equilibrium (forces and moments balance). There exists no stable equilibrium position for this part in a vector field with a simple squeeze pattern. 
A. regular polygons have $n$ stable orientation equilibria in a squeeze field; and

B. every connected polygon has $O\left(k n^{2}\right)$ stable orientation equilibria in a squeeze field.

\section{Proof.}

A. Because of their part symmetry, regular polygons have $2 n$ equilibria. Half of them are stable, the other $n$ are unstable.

B. See Section 3.2.

As described in Section 3.2, there exist simple polygons with $n$ vertices that can be bisected by a straight line in up to $O\left(k n^{2}\right)$ topologically different ways (Böhringer et al. 1997a). This suggests that there could be parts that have $\Omega\left(k n^{2}\right)$ orientation equilibria in a squeeze field, which would imply alignment strategies of length $\Omega\left(k n^{2}\right)$ and planning complexity $\Omega\left(k^{2} n^{4}\right)$.

While the counterexample in Figure 15 may be plausibly avoided by prohibiting parts with "point contacts," the other examples (Fig. 14 and Proposition 6) are more problematic. In Section 5, we show how to choose programmable vector fields that exclude some of these pathological behaviors, by using the theory of potential fields to describe a class of force vector fields for which all polygonal parts have stable equilibria. In Section 6.1, we show how to combine these fields to obtain new fields in which all parts have only $O(k n)$ equilibria.

We believe parts with point contact (not having finite area contact) will behave badly in all vector fields. We can model a point contact with delta functions, such that, e.g., for a pointcontact $P_{0}$ at $\left(x_{0}, y_{0}\right)$ :

$$
\int_{P_{0}} f d A=\int f \delta\left(x_{0}, y_{0}\right) d A=f\left(x_{0}, y_{0}\right) .
$$

This model is frequently used in mechanics (see, e.g., the work of Erdmann (1994)). Point contact permits rapid, discontinuous changes in force and moment. Hence, bodies with point contact will tend to exhibit instabilities, as opposed to flat parts that are in contact with a large number of (elastic) actuators. Finally, we believe that as the area contact-the size of the "feet" of a part-approaches zero, the part may become unstable. This represents a design constraint on parts that are to be manipulated using programmable planar parts feeders.

The lower bounds we demonstrate are indications of the pathologies that can arise when fields without potential or parts with point contact are permitted. Each of our counterexamples (Figs. 14 and 15) is "generic" in that it can be generalized to a very large class of similar examples. However, these lower bounds are just a first step, and one wishes for examples that delineate the capabilities of programmable vector fields for planar parts manipulation even more precisely.
The separating field shown in Figure 1c is not a potential field, and there exist parts that will spin forever, without equilibrium, in this field (this follows by generalizing the construction in Fig. 14). However, for specific parts, such as those shown in Figure 1, this field is useful if we can pose the parts appropriately first (e.g., using the potential field shown in Fig. lb).

Finally, we may "surround" nonpotential fields with potential fields to obtain reasonable behavior in some cases. Figure 1 shows how to "surround" a nonpotential field in time by potential fields, to eliminate pathologies. Similarly, we can surround nonpotential fields spatially. For example, if field lc could be surrounded by a larger potential field, then after separation, parts could reach a stable equilibrium.

Nonpotential fields can be used safely with the following methodology: let $H \subset \mathcal{C}=\mathbb{R}^{2} \times \mathbb{S}^{1}$ be the undesirable limit set. For example, $H$ could be a limit cycle where the part spins forever. Let $\hat{P}_{V}(H)$ be the weak pre-image (LozanoPérez, Mason, and Taylor 1984; Donald 1989) of $H$ under the field $V$. If we can ensure that the part starts in a configuration $z \notin \hat{P}_{V}(H)$, it will not reach the unwanted limit cycle. For example, in Figure 1 the centering step (b) ensures that the part does not end up on the border between the two separating fields, where it would spin forever in step (c).

\section{Completeness: Classification using Potential Fields}

We are interested in a general classification of all those vector fields in which every part has stable equilibria. As motivation, recall that a skewed vector field, even though very similar to a regular squeeze field (see again Fig. 7), induces no stable equilibrium in a disk-shaped part (Fig. 14). In this section, we discuss a family of vector fields that will be useful for manipulation tasks. These fields belong to a specific class of vector fields: the class of fields that have a potential.

We believe that fields without potential will often induce pathological behavior in many parts. Fields without potential admit paths along which a particle (point mass) will gain energy. Since mechanical parts are rigid aggregations of particles, this may induce unstable behavior in larger bodies. However, there are some cases where nonpotential fields may be useful. For example, see Figure lc, which is not a potential field. Such fields may be employed to separate but not to stabilize, pose, or orient parts. This strong statement devolves to our proof that fields like Figure 14 do not have well-behaved equilibria. Hence, they should only be employed when we want to induce an unstable system that will cast parts away from equilibrium, e.g., to sort or separate them.

Consider the class of vector fields on $\mathbb{R}^{2}$ that have a potential, i.e., fields $f$ in which the work is independent of the path, or equivalently, the work on any closed path is zero, $\oint f \cdot d s=0$. In a potential field, each point $(x, y)$ is assigned 
a real value $U(x, y)$ that can be interpreted as its potential energy. When $U$ is smooth, then the vector field $f$ associated with $U$ is the gradient $-\nabla U$. In general, $U(x, y)$ is given, up to an additive constant, by the path integral $\int_{a} f \cdot d s$ (when it exists and it is unique), where $\alpha$ is an arbitrary path from a fixed reference point $\left(x_{0}, y_{0}\right)$ to $(x, y)$. Assuming first-order dynamics, when $U$ is smooth, an ideal point object is in stable equilibrium when it is at a local minimum of $U$.

DEFINITION 6. Let $f$ be a force-vector field on $\mathbb{R}^{2}$, and let $\mathbf{p}$ be a point that is offset from a fixed reference point $\mathbf{q}$ by a vector $\mathbf{r}(\mathbf{p})=\mathbf{p}-\mathbf{q}$. We define the generalized force $F$ as the force and moment induced by $f$ at point $\mathbf{p}$ :

$$
F(\mathbf{p})=(f(\mathbf{p}), \mathbf{r}(\mathbf{p}) \times f(\mathbf{p}))
$$

Let $P$ be a part of arbitrary shape, and let $P_{z}$ denote the part $P$ in pose $\mathbf{z}=(x, y, \theta) \in \mathcal{C}$. We define the lifted force field $f_{P}$ as the area integral of the force induced by $f$ over $P_{\mathbf{z}}$ :

$$
f_{P}(\mathbf{z})=\int_{P_{\mathbf{z}}} f d A .
$$

The lifted generalized force field $F_{P}$ is defined as the area integral of the force and moment induced by $f$ over $P$ in configuration $\mathbf{z}$ :

$$
\begin{aligned}
F_{P}(\mathbf{z}) & =\int_{P_{\mathbf{z}}} F d A \\
& =\left(\int_{P_{\mathbf{z}}} f d A, \int_{P_{\mathbf{z}}} r \times f d A\right)
\end{aligned}
$$

Hence, $F_{P}$ is a vector field on $\mathcal{C}$. Finally, we define the lifted potential $U_{P}: \mathcal{C} \rightarrow \mathbb{R} . U_{P}$ is the area integral of the potential $U$ over $P$ in configuration $\mathbf{z}$ :

$$
U_{P}(\mathbf{z})=\int_{P_{\mathbf{z}}} U d A .
$$

We now show that the category of potential fields is closed under the operation of lifting, and that $U_{P}$ is the potential of $F_{P}$ (see Fig. 16). Note that $U$ need not be smooth.

Let $g: X \rightarrow Y$ and $h: Y \rightarrow Z$. Let $k: X \rightarrow Z$ be the function that is the composition of $g$ and $h$, defined by $k(x)=h(g(x))$. In the following proposition, we use the notation $h(g)$ to denote $k$, the function composition of $g$ and $h$.

PROPOSITION 7. Let $f$ be a force field on $\mathbb{R}^{2}$ with potential $U$, and let $P$ be a part of arbitrary shape. For the lifted generalized force field $F_{P}$ and the lifted potential $U_{P}$, the following equality holds: $U_{P}=\int_{P} U d A=\int_{\alpha} F_{P} \cdot d \mathbf{z}+c$, where $\alpha$ is

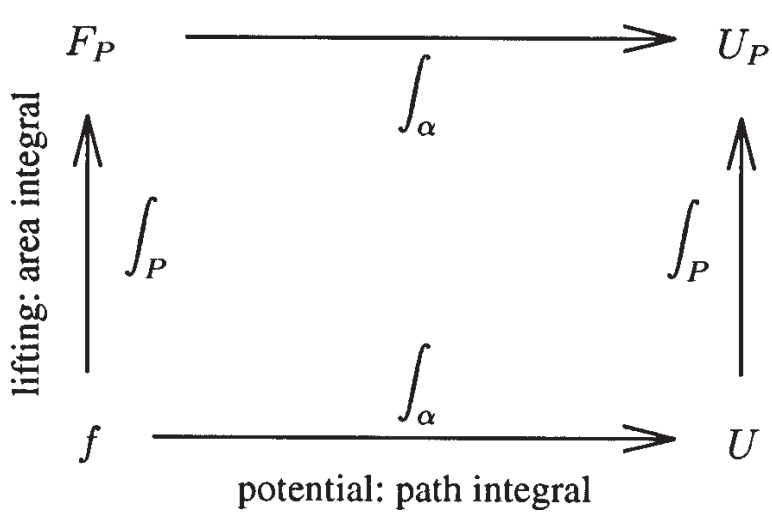

Fig. 16. Determining the potential, and lifting are commutative operations on force-vector fields.

an arbitrary path in $\mathcal{C}$ from a fixed reference point, and $c$ is a constant.

Proof. Given a force field $f$ with potential $U$, and a part $P$, we define $P^{*}$ as the set $\{(r, \eta) \mid(r \cos \eta, r \sin \eta) \in P\} \subset$ $\mathbb{R} \times \mathbb{S}^{1} . P^{*}$ is a representation of $P$ in polar coordinates: $\mathbf{p}$ $=(r \cos \eta, r \sin \eta) \in P$ if and only if $(r, \eta) \in P^{*}$.

We write $P_{\mathbf{z}}$ to denote $P$ in pose $\mathbf{z}=\left(x_{\mathbf{z}}, y_{\mathbf{z}}, \theta_{\mathbf{z}}\right)$. If $P$ is moved into pose $\mathbf{z}$, then the point $\mathbf{p}$ moves to $\mathbf{p}_{\mathbf{z}}=\left(x_{\mathbf{z}}+\right.$ $\left.r \cos \left(\theta_{\mathbf{z}}+\eta\right), y_{\mathbf{z}}+r \sin \left(\theta_{\mathbf{z}}+\eta\right)\right)=\left(x_{\mathbf{z}}, y_{\mathbf{z}}\right)+\mathbf{r}_{\mathbf{z}}$. Let us assume that for a given $P$, the COM of $P$ is at $\mathbf{0}$; this implies that the COM of $P_{\mathbf{z}}$ lies at $\left(x_{\mathbf{z}}, y_{\mathbf{z}}\right)$.

We define three families of functions $\rho, \zeta$, and $\alpha$, as follows:

$$
\rho_{r, \eta}:[0,1] \rightarrow \mathbb{R}^{2}
$$

such that $\rho_{r, \eta}$ is a smooth path in $\mathbb{R}^{2}$ with $\rho_{r, \eta}(0)=\mathbf{0}$ and $\rho_{r, \eta}(1)=\mathbf{p}_{\mathbf{0}}=(r \cos \eta, r \sin \eta)$,

$$
\zeta_{\mathbf{z}}:[0,1] \rightarrow \mathbb{R}^{2} \times \mathbb{S}^{1}
$$

such that $\zeta_{\mathbf{z}}$ is a smooth path in $\mathbb{R}^{2} \times \mathbb{S}^{1}$ with $\zeta_{\mathbf{z}}(\mathbf{0})=\mathbf{0}$ and $\zeta_{\mathbf{z}}(1)=\mathbf{z}=\left(x_{\mathbf{z}}, y_{\mathbf{z}}, \theta_{\mathbf{z}}\right)$, and

$$
\begin{array}{rlcc}
\alpha_{r, \eta}: & \mathbb{R}^{2} \times \mathbb{S}^{1} & \rightarrow & \mathbb{R}^{2} \\
& (x, y, \theta) & \mapsto & (x+r \cos (\theta+\eta), \\
& & & y+r \sin (\theta+\eta) .
\end{array}
$$

So $\zeta_{\mathbf{z}}$ is an arbitrary smooth path from $\mathbf{0}$ to $\mathbf{z}$ in $\mathcal{C}$, and $\alpha_{r, \eta}\left(\zeta_{\mathbf{z}}\right)$ is a smooth path in $\mathbb{R}^{2}$ from $\mathbf{p}_{\mathbf{0}}=(r \cos \eta, r \sin \eta)$ to $\mathbf{p}_{\mathbf{z}}=\left(x_{\mathbf{z}}+r \cos \left(\theta_{\mathbf{z}}+\eta\right), y_{\mathbf{z}}+r \sin \left(\theta_{\mathbf{z}}+\eta\right)\right)$. Recall that $\alpha_{r, \eta}\left(\zeta_{\mathbf{z}}\right)$ is the function composition of $\zeta_{\mathbf{z}}$ and $\alpha_{r, \eta}$.

We are interested in the potential of $U$ at $\mathbf{p}_{\mathbf{z}}$. 
$U\left(\mathbf{p}_{\mathbf{z}}\right)=\int_{\beta} f \cdot d s$,

where $\beta$ is some path from $\mathbf{0}$ to $\mathbf{p}_{\mathbf{z}}$. The integral is path independent, because $f$ has potential $U$. Since we can choose any path, we choose a path $\beta$ that consists of two parts: (1) a path from $\mathbf{0}$ to the point $\mathbf{p}_{\mathbf{0}}$, and (2) a path from $\mathbf{p}_{\mathbf{0}}$ to $\mathbf{p}_{\mathbf{z}}$.

$=\int_{\rho_{r, \eta}} f \cdot d s+\int_{\alpha_{r, \eta}\left(\zeta_{\mathbf{z}}\right)} f \cdot d s$

where the path $\alpha_{r, \eta}\left(\zeta_{\mathbf{z}}\right)$, given by the composition of $\zeta_{\mathbf{z}}$ and $\alpha_{r, \eta}$, depends on $\mathbf{z}$ as well as on $r$ and $\eta$, but $\rho_{r, \eta}$ is independent of $\mathbf{z}$. The left integral is the potential difference between $\mathbf{p}_{\mathbf{0}}$ and $\mathbf{0}$. Without loss of generality, let us choose $U(\mathbf{0})=0$.

$$
=U\left(\mathbf{p}_{\mathbf{0}}\right)+\int_{\zeta_{\mathbf{z}}} f\left(\alpha_{r, \eta}\right) \cdot(\mathbf{J} d \mathbf{z}),
$$

where $\mathbf{J}$ is the Jacobian:

$$
\mathbf{J}=\left(\begin{array}{ccc}
\frac{\partial \alpha x}{\partial x} & \frac{\partial \alpha x}{\partial y} & \frac{\partial \alpha x}{\partial \theta} \\
\frac{\partial \alpha y}{\partial x} & \frac{\partial \alpha y}{\partial y} & \frac{\partial \alpha y}{\partial \theta}
\end{array}\right)=\left(\begin{array}{ccc}
1 & 0 & -r \sin (\theta+\eta) \\
0 & 1 & r \cos (\theta+\eta)
\end{array}\right),
$$

which is the derivative of $\alpha_{r, \eta} . f\left(\alpha_{r, \eta}\right)$ is the function composition of $\alpha_{r, \eta}$ and $f$. Also note that $d \mathbf{z}=$ $(d x, d y, d \theta)$.

$$
\begin{aligned}
= & U\left(\mathbf{p}_{\mathbf{0}}\right)+\int_{\zeta_{\mathbf{z}}}\left(f_{x}\left(\alpha_{r, \eta}\right), f_{y}\left(\alpha_{r, \eta}\right),\right. \\
& \left.r \cos (\theta+\eta) f_{y}\left(\alpha_{r, \eta}\right)-r \sin (\theta+\eta) f_{x}\left(\alpha_{r, \eta}\right)\right) \cdot d \mathbf{z} . \\
= & U\left(\mathbf{p}_{\mathbf{0}}\right)+\int_{\zeta_{\mathbf{z}}} F\left(\alpha_{r, \eta}\right), \cdot d \mathbf{z} .
\end{aligned}
$$

Equation (16) states that the potential at a point $\mathbf{p}_{\mathbf{z}}=\left(x_{\mathbf{z}}+\right.$ $\left.r \cos \left(\theta_{\mathbf{z}}+\eta\right), y_{\mathbf{z}}+r \sin \left(\theta_{\mathbf{z}}+\eta\right)\right)$ can be determined as the sum of two integrals: the first integrates the force $f$ over a path from $\mathbf{0}$ to $\mathbf{p}_{\mathbf{0}}=(r \cos \eta, r \sin \eta)$. If we choose $U(\mathbf{0})=0$, then the first integral is the potential at point $\mathbf{p}_{\mathbf{0}}$. The right part of the expression can be understood as the path integral of the generalized force from $\mathbf{p}_{\mathbf{0}}$ to $\mathbf{p}_{\mathbf{z}}$

With this result, we can now consider the lifted potential $U_{P}$ at a point $\mathbf{z}=\left(x_{\mathbf{z}}, y_{\mathbf{z}}, \theta_{\mathbf{z}}\right) \in \mathcal{C}$ :

$$
\begin{aligned}
U_{P}(\mathbf{z})= & \int_{P_{\mathbf{z}}} U(\mathbf{p}) d A, \\
= & \iint_{P^{*}} U\left(\mathbf{p}_{\mathbf{z}}\right) r d r d \eta, \\
& \text { where } \mathbf{p}_{\mathbf{z}}=\left(x_{\mathbf{z}}+r \cos \left(\theta_{\mathbf{z}}+\eta\right), y_{\mathbf{z}}\right. \\
& \left.+r \sin \left(\theta_{\mathbf{z}}+\eta\right)\right) \operatorname{such} \text { that }(r, \eta) \in P^{*} . \\
= & \iint_{P^{*}}\left(U\left(\mathbf{p}_{\mathbf{0}}\right)+\int_{\zeta_{\mathbf{z}}} F\left(\alpha_{r, \eta}\right) \cdot d \mathbf{z}\right) r d r d \eta,
\end{aligned}
$$

by using eq. (16). Again, $F\left(\alpha_{r, \eta}\right)$ denotes the function composition of $\alpha_{r, \eta}$ and $F$.

$=\iint_{P^{*}} U\left(\mathbf{p}_{\mathbf{0}}\right) r d r d \eta$

$+\iint_{P^{*}}\left(\int_{\zeta \mathbf{z}} F\left(\alpha_{r, \eta}\right) \cdot d \mathbf{z}\right) r d r r \eta$.

The first expression is the area integral of $U$ over $P$. From Definition 6, it follows that this expression is equal to $U_{P}(\mathbf{0})$ (note that $U_{P}(\mathbf{0})$ is a constant that does not depend on $\mathbf{z})$.

$=U_{P}(\mathbf{0})$

$+\iint_{P^{*}}\left(\int_{0}^{1} F\left(\alpha_{r, \eta}\left(\zeta_{\mathbf{z}}(t)\right)\right) \cdot \zeta_{\mathbf{z}}^{\prime}(t) d t\right) r d r r \eta$,

where $\zeta_{\mathbf{z}}^{\prime}$ is the derivative of $\zeta_{\mathbf{z}}$. The dot product yields a scalar value. We can now switch the integrals.

$=\int_{0}^{1}\left(\iint_{P^{*}} F\left(\alpha_{r, \eta}\left(\zeta_{\mathbf{z}}(t)\right)\right) \cdot \zeta_{\mathbf{z}}^{\prime}(t) r d r d \eta\right) d t$ $+U_{P}(\mathbf{0})$.

$\zeta_{\mathbf{z}}^{\prime}$ is constant with respect to the integration parameters $r$ and $\eta$ hence, we can move $\zeta_{\mathbf{z}}^{\prime}$ outside of the area integral.

$=\int_{0}^{1}\left(\iint_{P^{*}} F\left(\alpha_{r, \eta}\left(\zeta_{\mathbf{z}}(t)\right)\right) r d r d \eta\right) \cdot \zeta_{\mathbf{z}}^{\prime}(t) d t$

$+U_{P}(\mathbf{0})$

$=\int_{0}^{1}\left(\iint_{P^{*}} F\left(\zeta_{\mathbf{z}, x}(t)+r \cos \left(\zeta_{\mathbf{z}, \theta}(t)+\eta\right)\right.\right.$, 


$$
\begin{aligned}
& \left.\left.\zeta_{\mathbf{z}, y}(t)+r \sin \left(\zeta_{\mathbf{z}, \theta}(t)+\eta\right)\right) r d r d \eta\right) \cdot \zeta_{\mathbf{z}}^{\prime}(t) d t \\
+ & U_{P}(\mathbf{0}), \\
& \text { where } \zeta_{\mathbf{z}}=\left(\zeta_{\mathbf{z}, x}, \zeta_{\mathbf{z}, y}, \zeta_{\mathbf{z}, \theta}\right) . \\
= & \int_{0}^{1}\left(\iint_{P_{\zeta_{\mathbf{z}}(t)}^{*}} F(r \cos \eta, r \sin \eta) r d r d \eta\right) \cdot \zeta_{\mathbf{z}}^{\prime}(t) d t \\
+ & U_{P}(\mathbf{0}), \\
& \text { where } P_{\zeta_{\mathbf{z}}(t)}^{*}=\left\{(r, \eta) \mid(r \cos \eta, r \sin \eta) \in P_{\left.\zeta_{\mathbf{z}}(t)\right\} .}\right. \\
= & \int_{0}^{1}\left(\int_{P_{\zeta_{\mathbf{z}}(t)}} F d A\right) \cdot \zeta_{\mathbf{z}}^{\prime}(t) d t+U_{P}(\mathbf{0}), \\
= & \int_{0}^{1} F_{P}\left(\zeta_{\mathbf{z}}(t)\right) \cdot \zeta_{\mathbf{z}}^{\prime}(t) d t+U_{P}(\mathbf{0}) \\
& \text { by definition of the lifted force } F_{P} . \\
= & \int_{\zeta_{\mathbf{z}}} F_{P} \cdot d \mathbf{z}+U_{P}(\mathbf{0}) .
\end{aligned}
$$

Hence, $U_{P}$ is indeed the potential of $F_{P}$.

We believe that a shorter proof is possible by using differential forms for the case that both $f$ and $U$ are smooth. However, since the fields in consideration are usually not smooth (e.g., unit squeeze or radial fields), we give the longer general proof here. Note that this proof does not rely on the fact that $f$ is a vector field on $\mathbb{R}^{2}$. Therefore, the proof generalizes to dimensions 3 or higher.

COROLLARY 2. Let $f$ be a force field on $\mathbb{R}^{2}$ with potential $U$, and let $P$ be a part of arbitrary shape. For the lifted generalized force $F_{P}$ and the lifted potential $U_{P}$, the following equality holds if $U_{P}$ is differentiable: $\nabla U_{P}=-F_{P}$.

Proof. Follows directly from Proposition 7.

So again, $U_{P}(x, y, \theta)$ can be interpreted as the potential energy of part $P$ in configuration $(x, y, \theta)$ Therefore, we obtain a lifted potential field $U_{P}$ whose local minima are the stable equilibrium configurations in $\mathcal{C}$ for part $P$. Furthermore, potential fields are closed under addition and scaling. We can thus create and analyze more complex fields by looking at their components. In general, the theory of potential fields allows us to classify manipulation strategies with vector fields, offering new insights into equilibrium analysis and providing the means to determine strategies with stable equilibria. For example, it allows us to show that orientation equilibrium

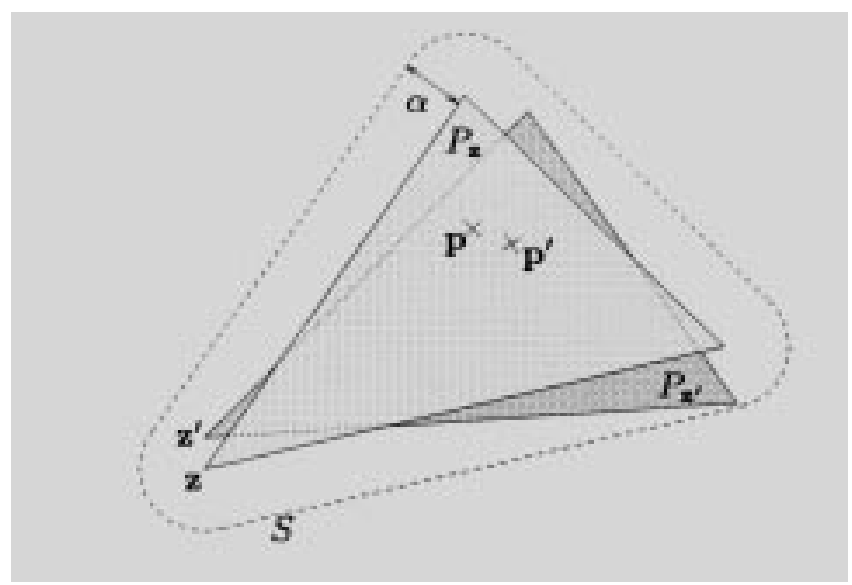

Fig. 17. Two triangles $P_{\mathbf{z}}$ and $P_{\mathbf{z}^{\prime}}$ with reference points $\mathbf{z}$ and $\mathbf{z}^{\prime}$ whose symmetric difference is less than $\epsilon$.

in a simple squeeze field is equivalent to the stability of a homogeneous boat floating in water, provided its density is $\rho=\frac{1}{2} \rho_{\text {water }}$ (for references on boat stability, see the works of Gillmer (1956, pp. 42ff) or Newman (1977, p. 290ff)).

\subsection{Properties of Lifted Force and Potential Fields}

In this section, we show that for a polygonal part $P$, the liftedforce field is always continuous, and the lifted potential is always smooth.

Proposition 8. Consider a polygon $P$ at two configurations: $\mathbf{z}=(x, y, \theta)$ and $\mathbf{z}^{\prime}=\left(x^{\prime}, y^{\prime}, \theta^{\prime}\right), \mathbf{z}, \mathbf{z}^{\prime} \in \mathcal{C}=\mathbb{R}^{2} \times \mathbb{S}^{1}$. For all $\epsilon>0$, there exists $a \delta>0$ such that if $\mathbf{z}^{\prime}$ lies within a $\delta$-ball around $\mathbf{z}, \mathbf{z}^{\prime} \in B_{\delta}(\mathbf{z})$, then $\mu\left(P_{\mathbf{z}} \Delta P_{\mathbf{z}^{\prime}}\right)<\epsilon(\mu(\cdot)$ denotes the size of an area, and $\Delta$ is the symmetric difference of two sets).

Proof. First we will create a region $S$ around $P_{\mathbf{z}}$ such that for any perturbed triangle $P_{\mathbf{z}^{\prime}} \subset S$, the nonoverlapping regions of $P_{\mathbf{z}}$ and $P_{\mathbf{z}^{\prime}}$ are less than a given $\epsilon$ in size. Then we will show that there always exists a region $B_{\delta}(\mathbf{z})$ around $\mathbf{z}$ such that if $\mathbf{z}^{\prime} \in B_{\delta}(\mathbf{z})$, then $P_{\mathbf{z}^{\prime}}$ lies in $S$.

For now, let us assume that $P_{\mathbf{z}}$ is a triangle in configuration $\mathbf{z}$, and let $a$ be the length of its longest side. Consider the set $S=P_{\mathbf{z}} \oplus B_{\alpha}(\mathbf{0})$, for some $a>0$ (Fig. 17). The area of $S-P_{\mathbf{Z}}$ is $\mu\left(S-P_{\mathbf{Z}}<3 \alpha(a+2 \alpha)\right.$. Let us choose $\alpha<\frac{1}{9}$ $\min (1, \epsilon, \epsilon / a)$. Then, if $\epsilon<1, \mu\left(S-P_{\mathbf{z}}\right)<\frac{1}{3}\left(\epsilon+2 \epsilon^{2}\right)<\epsilon$. If $\epsilon \geq 1, \mu\left(S-P_{\mathbf{Z}}\right)<\frac{1}{3}(\epsilon+2)<\epsilon$. So in both cases, if $P_{\mathbf{z}^{\prime}} \subset S$, then the area of the symmetric difference, $P_{\mathbf{z}} \Delta P_{\mathbf{z}^{\prime}}$ is at most $\epsilon$.

We are interested in the distance between a point $\mathbf{p} \in P_{\mathbf{z}}$, and the corresponding perturbed point $\mathbf{p}^{\prime} \in P_{\mathbf{z}^{\prime}}$. We can describe the points $\mathbf{p}$ and $\mathbf{p}^{\prime}$ as $\mathbf{p}=(x+r \cos (\phi+\theta), y+$ $r \sin (\phi+\theta))$ and $\mathbf{p}^{\prime}=\left(x^{\prime}+r \cos \left(\phi+\theta^{\prime}\right), y^{\prime}+r \sin \left(\phi+\theta^{\prime}\right)\right)$, where $r$ and $\phi$ are the length and the angle of a line from the reference point of $P$ to the point $\mathbf{p}$. The distance between the 
$x$-coordinates is

$$
\begin{aligned}
\left|x_{\mathbf{p}}-x_{\mathbf{p}^{\prime}}\right| & =\left|x+r \cos (\phi+\theta)-x^{\prime}-r \cos \left(\phi+\theta^{\prime}\right)\right| \\
& \leq\left|x-x^{\prime}\right|+\left|2 r \sin \frac{2 \phi+\theta+\theta^{\prime}}{2} \sin \frac{\theta-\theta^{\prime}}{2}\right| \\
& \leq\left|x-x^{\prime}\right|+r\left|\theta-\theta^{\prime}\right| .
\end{aligned}
$$

If we choose $\delta=\frac{\alpha}{4 \max (1, r)}$, we obtain

$$
\begin{aligned}
\left|x_{\mathbf{p}}-x_{\mathbf{p}^{\prime}}\right| & \leq \alpha / 4+\alpha / 4 \\
& =\alpha / 2 .
\end{aligned}
$$

Similarly, $\mid y_{\mathbf{p}}-y_{\mathbf{p}^{\prime}}<\alpha / 2$, and hence $\left|\mathbf{p}-\mathbf{p}^{\prime}\right|<\alpha$. We conclude that $\mathbf{p}^{\prime} \in S$ whenever $\mathbf{z}^{\prime} \in B_{\delta}(\mathbf{z})$. Hence we can always find a $\delta$-ball around $\mathbf{z}$ such that the areas of $P_{\mathbf{z}}$ and $P_{\mathbf{z}^{\prime}}$ differ by at most $\epsilon$ (by choosing $\delta<\frac{\alpha}{4 \max (1, r)}$, i.e., $\left.\delta<\frac{\min (1, \epsilon, \epsilon / a)}{36 \max (1, r)}\right)$.

This proof generalizes to arbitrary polygons (e.g., by using triangulations).

Proposition 9. Let $P$ be a polygonal part in a force field $f$ with potential $U$. The lifted force field $f_{P}(\mathbf{z})$, and the lifted generalized force field $F_{P}(\mathbf{z})$, with $\mathbf{z}=(x, y, \theta) \in \mathcal{C}=$ $\mathbb{R}^{2} \times \mathbb{S}^{1}$, are continuous functions in $x, y$, and $\theta$.

Proof. For a given $\gamma>0$, we want to determine an upper bound on the difference between $F(\mathbf{z})$ and $F\left(\mathbf{z}^{\prime}\right)$ for an $\operatorname{arbitrary} \mathbf{z}^{\prime} \in B_{\gamma}(\mathbf{z})$ :

$$
\begin{aligned}
\left|F(\mathbf{z})-F\left(\mathbf{z}^{\prime}\right)\right| & =\left|\int_{P_{\mathbf{z}}} f d A-\int_{P_{\mathbf{z}^{\prime}}} f d A\right|, \\
& \leq\left|\int_{P_{\mathbf{z}}-P_{\mathbf{z}^{\prime}}} f d A\right|+\left|\int_{P_{\mathbf{z}^{\prime}}-P_{\mathbf{z}}} f d A\right|, \\
& \leq \hat{f}\left(\int_{P_{\mathbf{z}}-P_{\mathbf{z}^{\prime}}} d A+\int_{P_{\mathbf{z}^{\prime}}-P_{\mathbf{z}}} d A\right), \\
& =\hat{f}\left|P_{\mathbf{z}} \Delta P_{\mathbf{z}^{\prime}}\right|,
\end{aligned}
$$

where $\hat{f}=\sup _{S}(|f(x, y)|)$ with $S=\left\{s \in P_{\mathbf{z}^{\prime}} \mid \mathbf{z}^{\prime} \in B_{\gamma}(\mathbf{z})\right\}$. This supremum exists whenever $f$ is integrable; i.e., if $f_{P}$ exists.

From Proposition 8, we know that we can make the area of $P_{\mathbf{z}} \Delta P_{\mathbf{Z}^{\prime}}$ arbitrarily small, by choosing an appropriate $\delta$ ball around $\mathbf{z}$. In particular, we can force it to be less than $1 / f$. Hence we can ensure that $\left|F(\mathbf{z})-F\left(\mathbf{z}^{\prime}\right)\right|<\epsilon$ for any $\mathbf{z}^{\prime} \in B_{\delta}(\mathbf{z})$, and any $\epsilon>0$. This implies that $F$ is continuous in $\mathbf{z}=(x, y, \theta)$.

An analogous argument holds for the lifted generalized force $F_{P}$.

COROLlaRY 3. For a polygonal part $P$, the lifted potential field $U_{P}(\mathbf{z})=\int_{P_{\mathbf{z}}} U d A$ is $C^{1}$ (i.e., its derivative exists and is continuous). Moreover, $\nabla U_{P}(\mathbf{z})=-F_{P}(\mathbf{z})$, where $F_{P}$ is the lifted generalized force acting on $P$.

Proof. Because of Proposition 7, $U_{P}(\mathbf{z})=\int_{\alpha} F_{P} \cdot d \mathbf{z}+c$ for some constant $c$. From Proposition 9, we know that the lifted generalized force $F_{P}$ is continuous; hence the path integral of $F_{P}$ must be $C^{1} . \nabla U_{P}(\mathbf{z})=-F_{P}(\mathbf{z})$ because of Corollary 2 .

\subsection{Examples: Classification of Force Fields}

Example 1. (Radial Fields) A radial field is a vector field whose forces are directed toward a specific center point. It can be used to center a part in the plane. The field in Figure $\mathrm{lb}$ can be understood as a radial field with a rather coarse discretization using only four different force directions. Note that this field has a potential.

DEFINITION 7. A unit radial field $R$ is a two-dimensional force-vector field such that $R(\mathbf{z})=-\mathbf{z} /|\mathbf{z}|$ if $\mathbf{z}=0$, and $R(\mathbf{0})=\mathbf{0}$.

Note that $R$ has a discontinuity at the origin. A smooth radial field can be defined; for example, by $R^{\prime}(\mathbf{z})=-\mathbf{z}$.

Proposition 10. Given the radial fields $R$ and $R^{\prime}$, the corresponding potential fields are $U(\mathbf{z})=\|\mathbf{z}\|$, and $U^{\prime}(\mathbf{z})=$ $\frac{1}{2}\|\mathbf{z}\|^{2}$, respectively.

Note that $U$ is continuous (but not smooth), while $U^{\prime}$ is smooth.

Counterexample 1 (Skewed Squeeze Fields): Consider again the skewed squeeze field in Figure 14. Note that, for example, the integral on a cyclic path along the boundary of the disk is nonzero. This explains why the disk-shaped part has no equilibrium.

Proposition 11. No skewed squeeze field has a potential.

Counterexample 2 (Parts with Point Contacts): Consider again the globally unstable $\mathrm{S}$-shaped part $P_{S}$ from Section 4 (Fig. 15). At first glance, this example may seem counterintuitive. It can be shown (see Lemma 4) that there must exist a pose $\mathbf{z}_{\min }$ in which $P_{S}$ achieves minimal potential, so why is $P_{S}$ not stable in pose $\mathbf{z}_{\min }$ ? To better understand this problem, we investigate $S$-shaped parts with finite area contacts, and the transition as their contact areas are decreased towards 0 .

Let us consider an S-shaped part with four square "feet." We choose the reference point at the COM, such that two of the feet are centered at $\pm\left(r_{A}, 0\right)$, and the other two feet are at $\pm\left(r_{B} \cos \phi, r_{B} \sin \phi\right)$ with $\phi$ constrained to $-\pi / 2<$ $\phi<\pi / 2$ (Fig. 18). Figure 19 shows two equilibria for an $\mathrm{S}$-shaped part. It is easy to see that these are the only two total equilibria, and that one of them (Fig. 19a) is unstable. For the following discussion, it is sufficient to investigate the behavior in a squeeze field with its reference point fixed at $(0,0)$.

Figure 20a shows the moment function $M_{P_{S}}$ and the potential $U_{P_{S}}$ of an S-shaped part, where $r_{A}=12, r_{B}=4, \phi=$ 


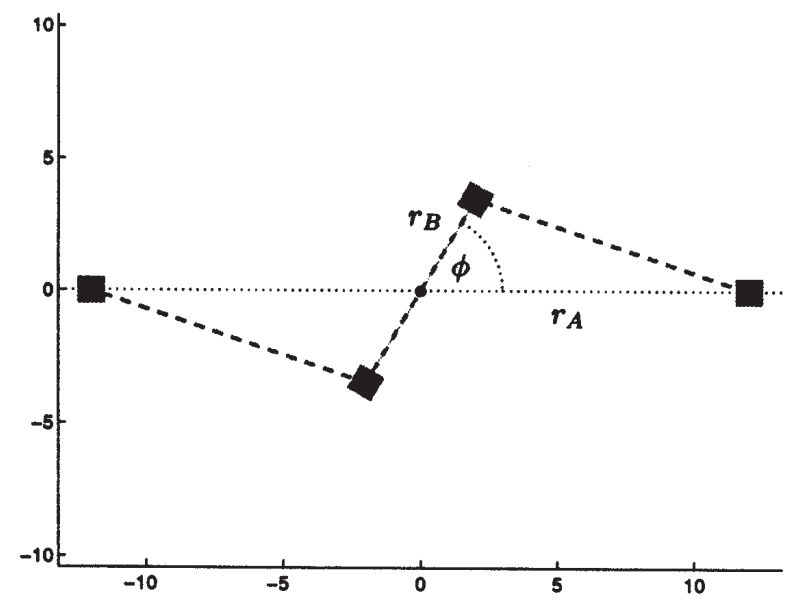

Fig. 18. An S-shaped part with four rigidly connected square "feet" in configuration $(x, y, \theta)=(0,0,0), r_{A}=12, r_{B}=$ 4 , and $\phi=60^{\circ}$.

$60^{\circ}$, and the feet have area size 10 . Notice that in poses with $\theta$-angles corresponding to minima in the potential, the moment has a root with negative slope, which indicates a stable (orientation) equilibrium. Figures $20 \mathrm{~b}$ and $20 \mathrm{c}$ show the (normalized) moments and potentials for parts with feet sizes 5 and 1, respectively. We observe that with decreasing contact areas, these functions become "less smooth," and the slope at the moment root increases. Figure 20d depicts moment and potential for a part with infinitesimally small feet. In this case, the moment function does not have a root at the minimum of the potential; rather, it exhibits a discontinuity at this orientation. This has the consequence that the part is not stable in this pose. In fact, for the moment function in Figure 20d, there exist no roots with negative slope, and hence there exists no stable equilibrium.

This observation can be made mathematically precise. The exact equations for the lifted potential and the moment of $P_{S}$ are

$$
\begin{aligned}
U_{P_{S}} & =2 r_{A}|\cos \theta|+2 r_{B}|\cos (\theta+\phi)|, \\
M_{P_{S}} & =2 r_{A} S(\theta)+2 r_{B} S(\theta+\phi), \\
\text { with } S(\theta) & = \begin{cases}\sin \theta & \text { if } 0 \leq \theta<\pi / 2 \text { or } 3 / 2 \pi<\theta<2 \pi, \\
-\sin \theta & \text { if } \pi / 2<\theta<3 / 2 \pi, \\
0 & \text { if } \theta=\pi / 2 \text { or } \theta=3 / 2 \pi .\end{cases}
\end{aligned}
$$

The potential minimum is reached at $\theta=\pi / 2$ or $\theta=$ $3 / 2 \pi$. However, we see that, for example, $M_{P_{S}}(\pi / 2)=$ $-2 r_{B} S(\pi / 2+\phi)=-2 r_{B} \cos \phi \neq 0$. Furthermore, $M_{P_{S}}(\pi / 2-)>0$, and $M(\pi / 2+)<0$. This implies that the part $P_{S}$ will oscillate about $\theta=\pi / 2$. Under first-order dynamics, this oscillation will be infinitesimally small, because any infinitesimal angular deflection of $P_{S}$ results in a restor-
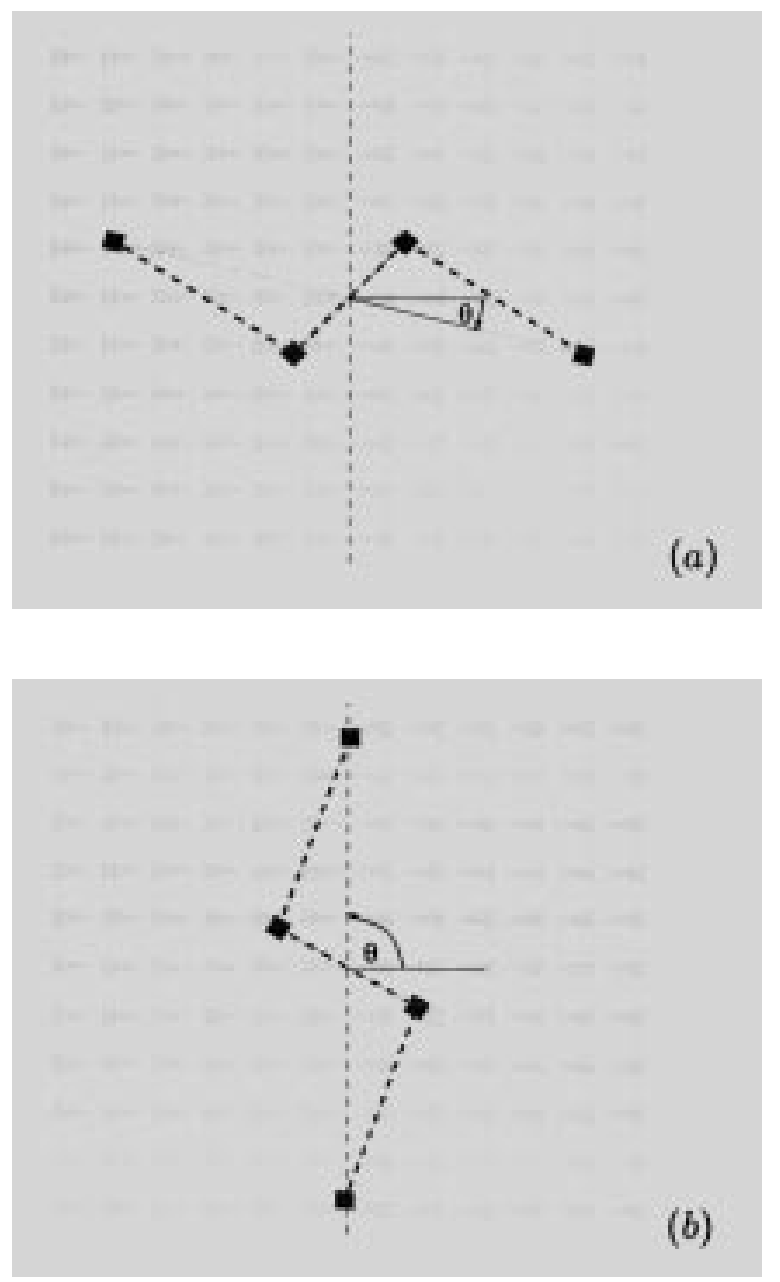

Fig. 19. Total equilibria of an S-shaped part with area contacts in a squeeze field. (a) Maximum potential, $\mathbf{z}_{\max }=$ $\left(0,0, \theta_{\max }\right)$, such that $r_{A} \sin \theta_{\max }=-r_{B} \sin \left(\theta_{\max }+\phi\right)$; $\theta_{\max } \approx-0.24$. (b) Minimum potential, $\mathbf{z}_{\min }=\left(0,0, \theta_{\min }\right)$; $\theta_{\min } \approx \pi / 2$.

ing moment with opposed orientation. Under second-order dynamics, the part may have a finite oscillation amplitude because of the inertia of the part. However, damping will reduce this amplitude over time.

We conclude that parts with point contacts can exhibit pathological behavior even in very simple and otherwise wellbehaved potential fields: this example shows that for such parts, it is possible that the generalized force is not zero in a pose that minimizes the potential of the part.

This pathology cannot occur when only parts with finite area contact are allowed. From Corollary 3, we know that the (lifted) potential of a part with area contact is $C^{1}$; hence its gradient exists everywhere. In particular, the gradient is zero at the minimum of the potential. This means that in a pose with minimum potential, the generalized force must be zero. Let us summarize these results. 


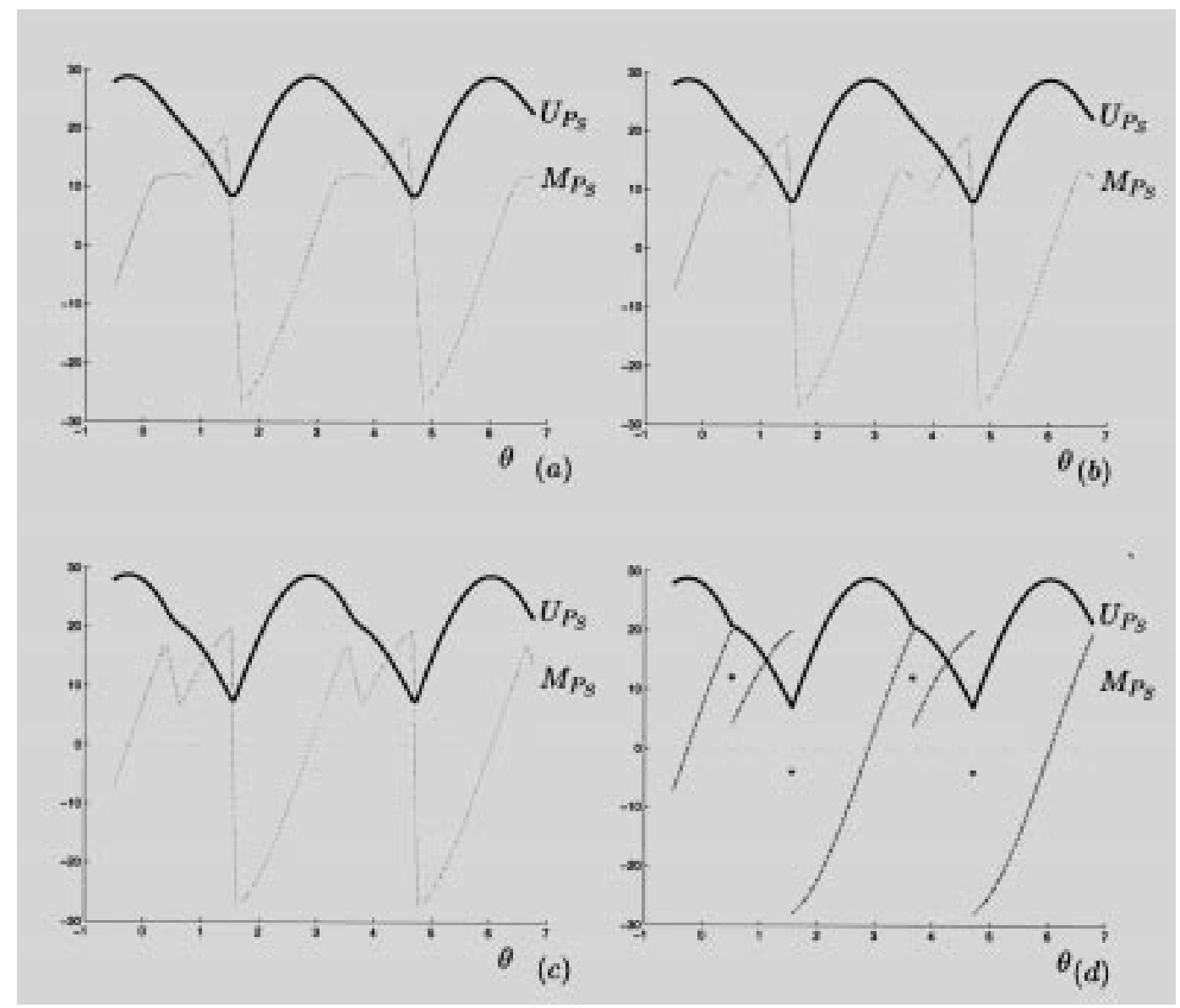

Fig. 20. Moment function $M_{P_{S}}$ (thin line) and potential $U_{P_{S}}$ (thick line) for S-shaped parts: (a) feet have contacts of area size 10 ; (b) size $=5$; (c) size $=1$; (d) point contacts. Note how a discontinuity is created in the moment function when the contact area is decreased toward 0 .

COROLlARY 4. Let $P$ be a part with finite area contact in a force-vector field $f$ with potential $U$. In a configuration $\mathbf{z}_{0}$ that corresponds to a local extremum of the lifted potential $U_{P}$, the lifted generalized force $F_{P}\left(\mathbf{z}_{0}\right)$ is zero.

In other words, for a first-order dynamical system and a part $P$ with finite-area contact, a local minimum (maximum) of $U_{P}$ corresponds to a stable (unstable) equilibrium of $P$ in $f$.

Example 2 (Morphing and Combining Vector Fields). Our strategies from Section 3 have switch points in time, where the vector field changes discontinuously (Fig. 7). This is because after one squeeze, for every part, the orientation equilibria form a finite set of possible configurations, but in general there exists no unique equilibrium (as shown in Section 3.3). Hence, subsequent squeezes are needed to disambiguate the part orientation. These switches are necessary for strategies with squeeze patterns.

One may ask whether, using another class of potential field strategies, unique equilibria may be obtained without discrete switching. We believe that continuously varying vector fields of the form $(1-t) f+t g$, where $t \in[0,1]$ represents time, and $f$ and $g$ are squeezes, may lead to vector fields that have this property. Here, "+" denotes point-wise addition of vector fields, and we will write "f $\rightsquigarrow g$ " for the resulting continuously varying field. By restricting $f$ and $g$ to be fields with potentials $U$ and $V$, we know that $U+V$ and $(1-t) U+t V$ are potential fields, and hence we can guarantee that $f+g$ and $f \rightsquigarrow g$ are well-behaved strategies. These form the basis of our new algorithms in Section 6.

Let us formalize the previous paragraphs. If $f$ is a vector field (in this case a squeeze pattern) that is applied to move part $P$, we define the equilibrium set $E_{P}(f)$ as the subset of the configuration space $\mathcal{C}$ for which $P$ is in equilibrium. Let us write $f * g$ for a strategy that first applies vector field $f$, and then vector field $g$, to move part $P . f+g$ can be understood as applying $f$ and $g$ simultaneously. We have shown that in general, $E_{P}(f)$ is not finite, but for two orthogonal squeezes $f$ and $g$, the discrete-switching strategy $f * g$ yields a finite equilibrium set $E_{P}(f * g)$ (see Section 6.2, Claim 1 ). Furthermore, for some parts, the equilibrium is unique up to symmetry. 
We wish to explore the relationship between equilibria in simple vector fields $E_{P}(f)$ or $E_{P}(g)$, combined fields $E_{P}(f+g)$, discretely switched fields $E_{P}(f * g)$, and continuously varying fields $E_{P}(f \rightsquigarrow g)$. For example, one may ask whether there exists a strategy with combined vector fields, or continuously varying fields, that, in just one step, reaches the same equilibrium as a discretely switched strategy requiring multiple steps. Finally, let $f_{1} * f_{2} * \ldots * f_{s}$ be a sequence of squeeze fields guaranteed to uniquely orient a part $P$ under assumption 2Phase. We wish to investigate how continuously varying strategies such as $f_{1} \rightsquigarrow f_{2} \rightsquigarrow \ldots \rightsquigarrow f_{s}$ can be employed to dynamically achieve the same equilibria even when the 2 Phase assumption is relaxed. The distributed actuation strategy $f * g$ is distributed in space, but not in time. The strategy $f+g$ is parallel with respect to space and time, since $f$ and $g$ are simultaneously "run." Research in this area could lead to a theory of parallel distributed manipulation that describes spatially distributed manipulation tasks that can be parallelized over time and space by superposition of controls.

\subsection{Upward-Shaped Potential Fields}

So far we have presented specific force fields that always (e.g., squeeze and radial fields) or never (e.g., skewed squeeze fields) induce stable equilibria on certain classes of parts. We conclude this section with a criterion that provides a sufficient condition on force fields such that all parts of a certain size reach a stable equilibrium.

We have observed in Section 4 that a priori it is not obvious when a force field induces stable equilibria. Our equilibrium criterion will be based on two important properties:

1. The field has a potential. Potential fields do not allow closed paths (technically, limit cycles) along which the work is positive, which could induce infinite motion of a part.

2. The force field is "inward-directed," which implies that (assuming first-order dynamics) parts can never leave a certain region, $R$. This useful property is a direct consequence of the definition of inward-directedness. An inward-directed force field corresponds to an "upwardshaped" potential, in which all paths that leave region $R$ have an ascending slope.

We will require Property 1 to hold for the entire force field, while Property 2 devolves to a boundary condition.

\subsubsection{Elementary Definitions}

DEFINITION 8. Let $\mathbf{z} \in \mathbb{R}^{n}$. The $\epsilon$-ball around $\mathbf{z}$, denoted $B_{\epsilon}(\mathbf{z})$, is the set $\left\{r \in \mathbb{R}^{n}|| \mathbf{r}-\mathbf{z} \mid<\epsilon\right\}$ of all points within a distance $\epsilon$ of $\mathbf{z}$.

DEFinition 9. (Lozano-Pérez 1983). Let $A, B$ be sets in $\mathbb{R}^{n}$. The Minkowski sum $A \oplus B$ of two sets $A$ and $B$ is defined as the set $\{\mathbf{a}+\mathbf{b} \mid \mathbf{a} \in \mathbf{A}, \mathbf{b} \in B\}$.
From these definitions, it follows that for a region $R$ with boundary $\partial R$, the set $\partial R \oplus B_{d}(\mathbf{0})=\{\mathbf{r}+\mathbf{z} \mid \mathbf{r} \in \partial R$, and $|\mathbf{z}| \leq d\}$ comprises all points that are within a distance $d$ from the boundary of $R$.

DEFINITION 10. Given a region $R \subset \mathbb{R}^{n}$, define the set $C I(R, d)=R-\left(\partial R \oplus B_{d}(\mathbf{0})\right.$, which is the region $R$ shrunk by distance $d$. Note that $C I(R, d)$ is based upon the configuration-space interior (Lozano-Pérez 1983) of $R$ for $B_{d}(\mathbf{0})$. Abusing terminology slightly, we call $C I(R, d)$ the configuration-space interior of $R$ in this paper.

DEFINITION 11. The radius $r_{P}$ of a part $P$ is the maximum distance between an arbitrary point of $P$ and the center of mass (COM) of $P$.

\subsubsection{Equilibrium Criterion}

We are now able to state a general criterion for a force field $f$ to induce stable equilibria on all parts in a region $S$. As mentioned at the beginning of Section 5.3, this criterion is based on two main conditions: (1) if $f$ has a potential, limit cycles with positive energy gain are avoided inside $S$; and (2) if $f$ is "inward-directed" (see the definition below), parts cannot leave the region $S$.

In the following, we give a general definition of inwarddirected vector fields on a manifold $Z$. We then specialize the definition to the special instances of $Z=\mathcal{C}=\mathbb{R}^{2} \times \mathbb{S}^{1}$ (the configuration space) and $Z=\mathbb{R}^{2}$, and give a sufficient, practical condition for inward-directed vector fields. We conclude with the presentation of our equilibrium criterion.

DEFINITION 12. (Inward-Directed Force Fields). ${ }^{5}$ Let $Z$ be an arbitrary smooth manifold, and let $Y \subset Z$ be a compact and smooth submanifold with boundary of $Z$. Assume that $\partial Y$ has codimension 1 in $Z$, and that the boundary of $Y$ is orientable. Let $q \in \partial Y$ be a point on the boundary of $Y$, and $V_{q} \in T_{q} Z$ be a tangent vector to $Z$ at $q$.

We say $V_{q}$ is inward-directed to $\partial Y$ at $q$ if there exists a sufficiently small $\epsilon>0$ such that $q+\epsilon V_{q} \in Y$.

Let $V$ be a vector field on $Z$. We say $V$ is inward-directed to $\partial Y$ if $V(q)$ is inward-directed to $\partial Y$ at $q$ for all $q \in \partial Y$.

Assume the set $S \subset \mathbb{R}^{2}$ is compact and smooth. Consider the part $P$ when it is placed into the force field $f$ such that its COM lies in $S$. The set of all such poses is a subset of the configuration space $\mathcal{C}=\mathbb{R}^{2} \times \mathbb{S}^{1}$, which we call $\tilde{S}=S \times \mathbb{S}^{1}$. The boundary of $\tilde{S}$ is $\partial \tilde{S}=\partial S \times \mathbb{S}^{1}$. Note that $\partial \tilde{S}$ separates the interior $i \tilde{S}=\tilde{S}-\partial S$ from the exterior $\left.\mathcal{C}-\tilde{S}=\mathbb{R}^{2}-S\right) \times \mathbb{S}^{1}$, and that $\partial \tilde{S}$ is isomorphic to a torus $\mathbb{S}^{1} \times \mathbb{S}^{1}$.

Now let $z=(x, y, \theta) \in \partial \tilde{S}$, and let $F_{z} \in T_{z} \mathcal{C}$ represent the lifted generalized force acting on part $P$ in pose $z . F_{z}$ is inward-directed (with respect to $\partial \tilde{S}$ ) if $F_{z}$ points into the interior of $\tilde{S}$. Note that this condition is equivalent to saying

5. In this definition, for convenience we assume that $Z$ is embedded in $\mathbb{R}^{m}$ for some $m$. This condition may be relaxed. 
that the projection of $F_{z}$ onto the tangent space at $(x, y)$ to $\mathbb{R}^{2}$ points into $S$, because the rotational component of $F_{z}$ is tangential to $\partial \tilde{S}$. So, for example, if $z=(x, y, \theta) \in \partial \tilde{S}$, then $z^{\prime}=\left(x, y, \theta^{\prime}\right) \in \partial \tilde{S}$ for any $\theta^{\prime}$.

The following proposition gives a simple condition on a force field $f$ that tells us if, for a given part $P$, its lifted generalized force field $F_{P}$ is inward-directed:

Proposition 12. Let $P$ be a part with radius $r$ whose COM is the reference point used to define its configuration space $C$ $=\mathbb{R}^{2} \times \mathbb{S}^{1}$. Let $f$ be a force-vector field defined on a region $R \subset \mathbb{R}^{2}$, with $F_{P}$ the corresponding lifted generalized force field. Let $S \subset \mathbb{R}^{2}$ be a convex, compact, and smooth subset of the configuration-space interior of $R$, and $S \subset C I(R, r)$.

Consider a point $q \in \partial S$ with outward normal $n_{q}$, and a ball $B_{r}(q)$ with radius $r$ about $q$. If for every point $q \in \partial S$, and for every point $s$ in the corresponding ball $B_{r}(q)$, the dot-product $g(s)=f(s) \cdot n_{q}$ is less than 0 , then the lifted generalized force field $F_{P}$ is inward-directed to $\partial S$ (note: $(\cdot)$ is the standard inner product).

Proof. Consider the part $P$ in pose $z=(x, y, \theta) \in \partial \tilde{S}$ such that $q=(x, y) . \quad P$ has radius $r$; hence it lies completely inside the ball $B_{r}(q)$, independent of its orientation $\theta$. As we know that $g(p)=f(p) \cdot n_{q}<0$ for all $p \in B_{r}(q)$, we can conclude that the integral of $g(p)$ over $P$ is also less than 0: $\int_{P} g(p) d A=\int_{P} f(p) \cdot n_{q} d A=f_{P} \cdot n_{q}<0$. This implies that for $f_{P}$, which is the translational component of $F_{P}$ (see Definition 6), the vector $q+\epsilon f_{P}(z)$ lies inside $S$, if $\epsilon$ is positive and sufficiently small. As mentioned above, this suffices to ensure that the vector $z+\epsilon F_{P}(z)$ lies inside $\tilde{S}$.[]

LEMMA 4. (Equilibrium Criterion). Let $P$ be a polygonal part with radius $r$, let $f$ be a force field with potential $U$ defined on a region $R \subset \mathbb{R}^{2}$, and let $S \subset R$ as specified in Proposition 12. Let us also assume that the motion of part $P$ is governed by first-order dynamics.

If the lifted force-vector field $F_{P}$ is inward-directed to $\partial \tilde{S}$, then the part $P$ will reach a stable equilibrium under $f$ in $i \tilde{S}$ whenever its COM is initially placed in $S$.

Proof. Assume that the COM of part $P$ is placed at a point $(x, y) \in S$. This means that $P$ is in some pose $z=(x, y, \theta) \in$ $\tilde{S}$. We now show that the COM of $P$ cannot leave $S$ when initially placed inside $S$. We know that $\partial \tilde{S}$ separates $i \tilde{S}$ from $\mathcal{C}-\tilde{S}$. Hence every path from $z$ to some $z^{*} \in \mathcal{C}-\tilde{S}$ must intersect $\partial \tilde{S}$ at some point $z^{\prime} \in \partial \tilde{S}$. Now consider part $P$ in pose $z^{\prime}$. Under first-order dynamics, its velocity must be in the direction of $F_{P}\left(z^{\prime}\right)$. Because $F_{P}$ is inward-directed, the velocity of $P$ must be toward $i \tilde{S}$. In particular, this means that the COM will move into $i S$; hence $P$ cannot leave $S$, and that there is no equilibrium on $\partial S$.

Because of Proposition 7, $f$, and hence $F_{P}$, have potential $U$ and $U_{P}$, respectively. Therefore limit cycles with energy gain are not possible. Furthermore, $U_{P}(\tilde{S})$ is the continuous image of a compact set, $\tilde{S}$. Therefore the image $U_{P}(\tilde{S})$ is a compact subset of $\mathbb{R}$, which has a minimum value attained by some point $s \in \tilde{S}$. Since $f$ is inward-directed, $s$ must lie in $i \tilde{S}$. This minimum is a stable equilibrium of $P$ in $f$, as shown in Corollary 4. []

Because of Lemma 4, the use of potential fields is invaluable for the analysis of effective and efficient manipulation strategies, as discussed in the following section. In particular, it is useful for proving the completeness of a manipulation planner.

\section{New and Improved Manipulation Algorithms}

The part-alignment strategies in Section 3.3 have switch points in time where the vector field changes discontinuously (Fig. 12). We can denote such a switched strategy by $f_{1} * f_{2} * \ldots * f_{s}$, where the $f_{i}$ are vector fields. In Section 3.3, we showed that a general squeeze strategy to align a (nonconvex) polygonal part with $n$ vertices may need up to $O\left(k n^{2}\right)$ switches, and require $O\left(k^{2} n^{4}\right)$ time in planning ( $k$ is the maximum number of polygon edges that a bisector can cross). To improve these bounds, we now consider a broader class of vector fields including simple squeeze patterns, radial, and combined fields, as described in Section 5.

In Section 6.1 we show how, by using radial and combined vector fields, we can significantly reduce the complexity of the strategies from that of Section 3. In Section 6.2 we describe a general planning algorithm that works with a limited "grammar" of vector fields (and yields, correspondingly, lessfavorable complexity bounds).

\subsection{Radial Strategies}

Consider a part $P$ in a force field $f$. Some force fields exhibit rotational symmetry properties that can be used to generate efficient manipulation strategies:

Property 1: There exists a unique pivot point $v$ of $P$ such that $P$ is in translation equilibrium if and only if $v$ coincides with $\mathbf{0 .}$

Property 2: $\quad$ There exists a unique pivot point $v$ of $P$ such that $P$ is in (neutrally stable) orientation equilibrium if and only if $v$ coincides with $\mathbf{0}$.

We typically think of the pivot point $v$ being a point of $P$; however, in generality, just like the center of mass of $P, v$ does not need to lie within $P$, but instead is some fixed point relative to the reference frame of $P$. Now consider the part $P$ in an ideal unit radial force-vector field $R$ as described in Section 5 .

Proposition 13. In a unit radial field $R$, Properties 1 and 2 hold.

Proof. First, we fix the part $P$ at an arbitrary orientation $\theta$, and show that at this orientation $P$ has a unique translation 
equilibrium $v(\theta)$. That is, placing $v(\theta)$ at the origin is necessary and sufficient for $P$ to be in translation equilibrium at orientation $\theta$. Second, we show that for any two distinct orientations $\theta$ and $\theta^{\prime}, v(\theta)=v\left(\theta^{\prime}\right)$. We call this unique point $v$, dropping the orientation $\theta$. Finally, we argue that whenever $P$ is in translation equilibrium (i.e., $v$ is at the origin), $P$ is neutrally stable with respect to orientation. This follows by the radial symmetry of $R$.

Consider the translational forces (but not the moments) acting on $P$ in the radial field $R$. To do this, let us separate $R$ into its $x$ - and $y$-components, $R_{x}$, and $R_{y}$, such that $R=\left(R_{x}, R_{y}\right)$. Assume for now that the orientation of $P$ is fixed. If $P$ is placed at a position $z_{0} \in \mathbb{R}^{2}$, whose $x$ coordinate is sufficiently negative, the total force induced by $R_{x}$ on $P$ will point in the positive $x$-direction. Symmetrically, placing $P$ at a sufficiently large positive $x$-coordinate will cause a force in the negative $x$-direction. We claim that by translating $P$ rigidly with an increasing $x$-coordinate, this force decreases continuously and strictly monotonically, and hence has a unique root.

To verify this claim, consider a small area patch $\mathcal{P}_{0}$ of $P$. A uniform translation $t$ of $\mathcal{P}_{0}$ in the $x$-direction can be described as $\mathcal{P}(t)=\mathcal{P}_{0} \oplus\left(z_{0}+t \hat{x}\right.$ ) (with $z_{0}$ the initial position of the patch, $\hat{x}$ the unit vector in the $x$-direction, and $\oplus$ the Minkowski sum). The total force on $\mathcal{P}(t)$ in the $x$ direction is $\int_{\mathcal{P}(t)} R_{x} d A$. This force decreases continuously and strictly monotonically with $t$, because $R_{x}$ is strictly monotone and continuous everywhere except on the $x$-axis, which has measure zero in $\mathbb{R}^{2}$. A similar argument applies for the $y$-direction, and because of the radial symmetry of $R$, for any direction.

If we choose the set $S$ as a sufficiently large disk-shaped region around the origin, and recall that $R$ has a potential, we can apply Lemma 4 to conclude that there must exist at least one total equilibrium for $P$. Now assume that there exist two distinct equilibria $e_{1}=\left(x_{1}, y_{1}, \theta_{1}\right)$, and $e_{2}=\left(x_{2}, y_{2}, \theta_{2}\right)$ for $P$ in $R$. We write " $P\left(e_{i}\right)$ " to denote that $P$ is in configuration $e_{i}$. Because of the radial symmetry of $R$, we can reorient $P\left(e_{2}\right)$ to $P\left(e_{2}^{\prime}\right)$ such that its orientation is equal to $P\left(e_{1}\right.$ : $e_{2}^{\prime}=\left(x_{2}^{\prime}, y_{2}^{\prime}, \theta_{1}\right)$, where $\left(\begin{array}{l}x_{2}^{\prime} \\ y_{2}^{\prime}\end{array}\right)=M\left(\begin{array}{l}x_{2} \\ y_{2}\end{array}\right)$, and $M$ is a rotation matrix with angle $\theta_{1}-\theta_{2}$ (Fig. 21). This reorientation does not affect the equilibrium. Note that $P$ can be moved from $e_{1}$ to $e_{2}^{\prime}$ by a pure translation. From above, we know that such a translation of $P$ corresponds to a strictly monotone change in the translational forces acting on $P$. Hence we conclude that $P\left(e_{1}\right)$ and $P\left(e_{2}^{\prime}\right)$ cannot both be in translation equilibrium unless $e_{1}$ and $e_{2}^{\prime}$ are equal. This implies that $e_{1}$ and $e_{2}$ cannot both be equilibria of $P$ in $R$ unless they both have the same pivot point $v$.

Definition 7 assumes that the center of a radial field lies at the origin. This definition can be generalized to radial fields with arbitrary centers $\left(x_{c}, y_{c}\right)$. Then properties 1 and 2 hold when the pivot point $v$ coincides with $\left(x_{c}, y_{c}\right)$. Surprisingly,

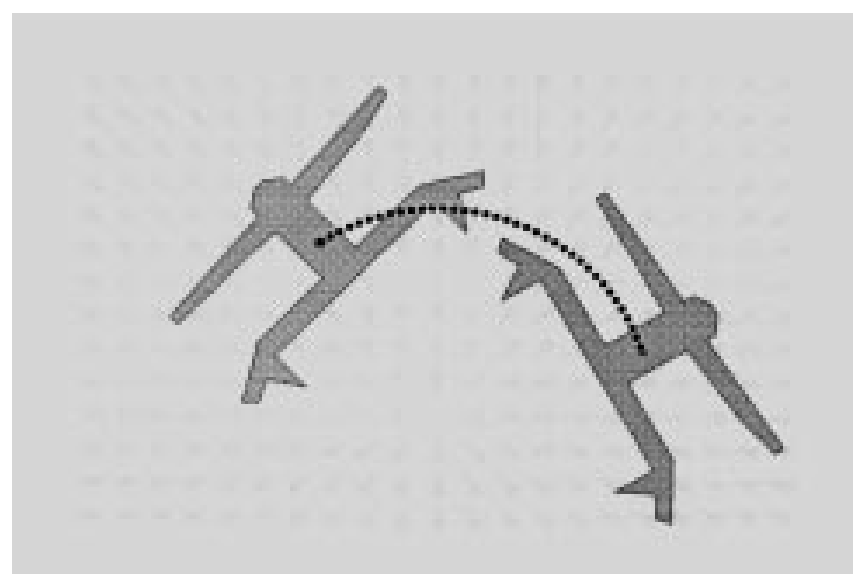

Fig. 21. Rotating a part about the center of a unit radial field. The force and torque on the part remain constant with respect to its reference frame.

$v$ need not be the center of area $P$ : for example, consider again the part in Figure 9, which consists of a large and a small square connected by a long rod of negligible width. The pivot point of this part will lie inside the larger square. But if the rod is long enough, the center of area will lie outside of the larger square. However, the following corollary holds:

COROLlARY 5. For a part $P$ in a continuous radial force field $R^{\prime}$ given by $R^{\prime}(\mathbf{z})=-\mathbf{z}$, the pivot point of $P$ coincides with the center of area of $P$.

Proof. The force acting on $P$ in $R^{\prime}$ is given by $F=\int_{P}-\mathbf{z} d A$, which is also the formula for the (negated) center of area. $\square$

Now suppose that $R$ is combined with a unit squeeze pattern $S$, which is scaled by a factor $\delta>0$, resulting in $R+\delta S$. The squeeze component $\delta S$ of this field will cause the part to align with the squeeze, similarly to the strategies in Section 3.3. But note that the radial component $R$ keeps the part centered in the force field. Hence, by keeping $R$ sufficiently large (or $\delta$ small), we can assume that the pivot point of $P$ remains within an $\epsilon$-ball of the center of $R$. This implies that assumption 2Phase (see Section 3.3) is no longer necessary. Moreover, $\epsilon$ can be made arbitrarily small by an appropriate choice of $\delta$.

PROPOSITION 13. Let $P$ be a polygonal part with $n$ vertices, and let $k$ be the maximum number of edges that a bisector of $P$ can cross. Let us assume that $v$, the pivot point of $P$, is in general position. There are at most $O(k n)$ stable equilibria in a field of the form $R+\delta S$ if $\delta$ is sufficiently small and positive.

Proof. For a part in equilibrium in a pure radial field $R$ (i.e., with $\delta=0$ ), the pivot point $v$ is essentially fixed at the origin. This is implied by Property 1 . It is easy to see that Property 1 is not true in general for arbitrary fields of the form $R+\delta S$. Property 1 holds if $\delta=0$, because then any orientation is an 
equilibrium when $v$ is at the center of $R$. However, Property 2 does not hold if $\delta>0$, because in general there does not exist a unique pivot point in squeeze fields (see Section 3.3).

We conduct the combinatorial analysis of the orientation equilibria under the assumptions that (1) $\delta>0$, and (2) that $v$ is fixed at the origin. Then we relax the latter assumption, and show that Property 1 holds, approximately, even in $R+\delta S$, for a sufficiently small $\delta>0$. That is, we show that a sufficiently small $\delta$ can be chosen so that the combinatorial analysis is unaffected when assumption (2) is relaxed.

First, we show that when $\delta$ is small but positive, and with $v$ fixed at the center of $R$, there are only a linear number of orientation equilibria (i.e., we constrain the pivot point $v$ to remain fixed at the origin until further notice). So let us assume that we are in a combined radial and small squeeze field $R+\delta S$.

Consider a ray $w(0)$ emanating from $v$. Assume without loss of generality that $v$ is not a vertex of $P$, and that $w(0)$ intersects the edges $S(0)=\left\{e_{1}, \ldots, e_{k}\right\}$ of $P$ in general position, $1 \leq k \leq n$. Parameterize the ray $w(\cdot)$ by its angle $\phi$ to obtain $w(\phi)$. As $\phi$ sweeps from 0 to $2 \pi$, each edge of $P$ will enter and leave the crossing structure $S(\phi)$ exactly once. $S(\phi)$ is updated at critical angles where $w(\phi)$ intersects a vertex of $P$. Since there are $n$ vertices, there are $O(n)$ critical angles, and hence $O(n)$ changes to $S(\phi)$ overall. Hence, since between critical angles $S(\phi)$ is constant, we see that $S(\phi)$ takes on $O(n)$ distinct values. Now place the squeeze line $l$ to coincide with $w(\phi)$. For a given crossing structure $S(\phi) \cup S(\phi+\pi)$, satisfying conditions 1 and 2 as defined in Section 3.3 devolves to solving two equations. The first equation provides the condition for translation equilibrium, while the second equation implements the condition for orientation equilibrium. The latter equation is called the moment function $M(\phi)$, because it describes the moment acting on $P$ as a function of $\phi$. (But note that $M$ is different from the moment function defined in Section 3.3, because here the part rotates about a fixed pivot point.) In analogy to Section 3.3, it can be shown that these equations are algebraic and of degree $k$, where $k$ is the maximum number of edges intersected by the squeeze line as described in Section 3.2. This implies that between any two adjacent critical values there are only $O(k)$ orientations of $l$ (given by $w(\phi))$ that satisfy conditions 1 and 2 . Hence, the overall number of orientations satisfying conditions 1 and 2 is $O(k, n)$.

If $\delta>0$, the part $P$ will be perturbed, so that Property 1 is only approximately satisfied. (That is, we can relax the assumption that $v$ is constrained to be at the origin). However, we can ensure that $v$ lies within an $\epsilon$-ball around the origin (the center of the radial field). To see this, first consider $P$ at some arbitrary configuration $\mathbf{z}$ in the squeeze field $\delta S$. The total squeeze force on $P_{\mathbf{z}}$ is given by the area integral $\delta S_{P}(\mathbf{z})=$ $\int_{P_{\mathbf{z}}} \delta S d A$. (Recall that $S_{P}$ denotes the lifted force field of $S$; see Definition 6, eq. (13).) Now $\delta S_{P}$ is bounded above by $\left|\delta S_{P}\right| \leq \delta A$, where $A$ is the area of $P$ (note that $S$ is a unit squeeze field).

$P$ is in equilibrium with respect to the radial field $R$ if $v$ is at the origin. Now consider the lifted force $R_{P}$ when the pivot point of $P$ is not at the origin. More specifically, let $v_{\mathbf{z}}$ be the pivot point of $P_{\mathbf{z}}$, and let us define a set $\mathcal{R}_{P}(d)=\left\{\left|R_{P}(\mathbf{z})\right|\right.$ such that $\left.\left|v_{\mathbf{z}}\right|=d\right\}$. We also define a function $\hat{R}_{P}(d)=$ min $\left\{\mathcal{R}_{P}(d)\right\}$. This function is well defined, because $\mathcal{R}_{P}(d)$ is the continuous image of a compact set; hence the minimum exists. $\hat{R}_{P}(d)$ is the minimum magnitude of the lifted force acting on $P_{\mathbf{z}}$ when its pivot point $v_{\mathbf{z}}$ is at distance $d$ from the origin.

By decomposing $R_{P}$ into its $x$ - and $y$-components, we can write $\left|R_{P}\right|$ as $\sqrt{R_{P, x}^{2}+R_{P, y}^{2}}$. Because of the radial symmetry of $R$, let us assume without loss of generality that $v_{\mathbf{z}}=(d, 0)$. From the proof of Proposition 13, we know that for any given orientation of $P_{\mathrm{z}}$, the magnitude of $R_{P, x}$ increases continuously and strictly monotonically with increasing $d \geq 0$. Furthermore, $R_{P, y}$ is continuous in $d$, and $R_{P, y}(\mathbf{0})=0$, so $R_{P, y}^{2}$ is continuous and monotonically increasing for all $d$ less than some sufficiently small $d_{0}>0$. Hence for any fixed orientation of $P_{\mathbf{z}}, R_{P}$ is a continuous and strictly monotonically increasing function for all $d \in\left|0, d_{0}\right|$.

Now suppose that $\hat{R}_{P}(d)$ is not strictly monotone, i.e., that there exist $d_{1}, d_{2}$ with $0 \leq d_{1}<d_{2}$, but $\hat{R}_{P}\left(d_{1}\right) \geq \hat{R}_{P}\left(d_{2}\right)$. Then there must exist $\mathbf{z}_{1}, \mathbf{z}_{2}$ with $\left|v_{\mathbf{z}_{1}}\right|=d_{1}$ and $\left|v_{\mathbf{z}_{2}}\right|=d_{2}$, and $\left|R_{P}\left(\mathbf{z}_{1}\right)\right|=\hat{R}_{P}\left(d_{1}\right) \geq \hat{R}_{P}\left(d_{2}\right)=\left|R_{P}\left(\mathbf{z}_{2}\right)\right|$. Let us define $\mathbf{z}_{2}^{\prime}$ such that $\mathbf{z}_{2, \theta}^{\prime}=\mathbf{z}_{2 \theta}$ and $v_{\mathbf{z}_{2}^{\prime}}=c v_{\mathbf{z}_{2}}$ for some $c \in \mathbb{R}$, i.e., $v_{\mathbf{z}_{2}}$ and $v_{\mathbf{z}_{2}^{\prime}}$ lie on a line through the origin. If we choose $0 \leq$ $c<1$, then $R_{P}\left(\mathbf{z}_{2}^{\prime}\right)|<| R_{P}\left(\mathbf{z}_{2}\right) \mid$, because $\left|R_{P}\right|$ is monotone, as shown in the previous paragraph. In particular, if we choose $c=d_{1} / d_{2}$, then $R_{P}\left(\mathbf{z}_{2}^{\prime}\right)|<| R_{P}\left(\mathbf{z}_{2}\right)|\leq| R_{P}\left(\mathbf{z}_{1}\right) \mid=\hat{R_{P}}\left(d_{1}\right)$, and $\left|v_{\mathbf{z}_{2}^{\prime}}\right|=\left|v_{\mathbf{z}_{1}}\right|$. This is a contradiction to the definition of $\hat{R_{P}}\left(d_{1}\right)=\min \left\{\mathcal{R}_{P}(d)\right\}$. We conclude that $R_{P}$ is continuous and strictly monotone for sufficiently small $d \geq 0$.

Now consider $P_{\mathbf{Z}}$ in the combined field $R+\delta \bar{S}$, and again let $d$ denote the distance between pivot point $v_{\mathbf{z}}$ and the origin. In equilibrium, the lifted forces $R_{P_{\mathbf{Z}}}$ and $\delta S_{P_{\mathbf{z}}}$ balance out; hence $\hat{R}_{P}(d) \leq\left|R_{P_{\mathbf{z}}}\right|=\left|\delta S_{P_{\mathbf{z}}}\right| \leq \delta A$, with $A$ the area of $P$. Since $\hat{R}_{P}$ is continuous and strictly monotone in $d$ for sufficiently small $d$, we can ensure that $d$ is less than a given $\epsilon$, by choosing an appropriately small $\delta$. This implies that $v_{\mathbf{z}}$ must lie within an $\epsilon$-ball of the center of the radial field. In particular, we can make this $\epsilon$-ball small enough so that the crossing structure $S(\phi)$ is not affected.

Finally, we have to ensure that the stable equilibria, as predicted by the moment function $M$, are approximated arbitrarily closely. This means that the disturbance in the moment function, caused by pivot point $v_{\mathbf{z}}$ not exactly coinciding with the center of the radial field, can be made arbitrarily small. To see this, first consider the original (unperturbed) moment function $M$, which describes the moment acting on the part $P$ if its pivot point coincides with the origin. In this case, the moment is caused solely by the squeeze field $\delta S$, while $P$ is 
in equilibrium with respect to the radial field $R$.

Now consider the disturbance in $M$ if the pivot point $v_{\mathbf{z}}$ is not exactly at the origin, but somewhere in an $\epsilon$-ball around it. Let us call this disturbance $\Delta M$, and note that $\Delta M$ has two components: $\Delta M_{\delta S}$, which is the change in moment caused by the squeeze field $\delta S$, and $\Delta M_{R}$, the disturbance caused by the radial field $R$.

For a part $P$ at a given orientation, any $\epsilon$-displacement of $v_{\mathbf{Z}}$ can change the force in a squeeze field $\delta S$ by at most $\left|\Delta F_{\delta S}\right| \leq \delta \epsilon d_{P}$, where $d_{P}$ is the maximum diameter of $P$. Hence $\Delta M_{\delta S}=r \times \Delta F_{\delta S}$ is proportional to the product of the disturbance in location $\epsilon$, and the magnitude of the squeeze field $\delta$ ( $r$ is the fixed distance between pivot point and COM of $P$ ), i.e., $\left|\Delta M_{\delta S}\right|=O(\delta \epsilon)$.

Since the force caused by the radial field $R$ balances the force generated by the squeeze field $\delta S$, we obtain the same bounds for $\Delta M_{R}$. We see that $\Delta M=\Delta M_{\delta S}+\Delta M_{R}=$ $O(\delta \epsilon)$. Recall that $\epsilon$ decreases strictly monotonically with $\delta$; hence $\Delta M$ decreases asymptotically faster than $\delta$. This ensures that we can find a sufficiently small $\delta$ such that the moment function $M$ is approximated arbitrarily closely, and the equilibria of the squeeze field $\delta S$ are not affected.

We conclude that the number of equilibria in a field $R+\delta S$ is bounded by $O(k n)$, for sufficiently small $\delta$.

In analogy to Section 3.3, we define the turn function $t: \mathbb{S}^{1} \rightarrow \mathbb{S}^{1}$, which describes how the part will turn under a squeeze pattern, and hence yields the stable equilibrium configurations. Given the turn function $t$, we can construct the corresponding squeeze function $s$ as described in Section 3.3. With $s$ as the input for the alignment planner described by Goldberg (1993), we obtain strategies for unique part alignment (and positioning) of length $O(k n)$. They can be computed in time $O\left(k^{2} n^{2}\right)$.

The result is a strategy for parts positioning of the form $\left(R+\delta S_{1}\right) * \cdots *\left(R+\delta S_{O(k n)}\right)$. Compared to the general squeeze algorithm in Section 3.3, it improves the plan length by a factor of $n$, and the planning complexity is reduced by a factor of $n^{2}$. The planner is complete: for any polygonal part, there exists a strategy of the form $*_{i}\left(R+\delta S_{i}\right)$. Moreover, the algorithm is guaranteed to find a strategy for any input part. By appending a step that is merely the radial field $R$ without a squeeze component, we are guaranteed that the part $P$ will be uniquely posed ( $v$ is at the origin) as well as uniquely oriented. We can also show that the continuously varying "morphing" strategy $\left(R+\delta S_{1}\right) \rightsquigarrow \cdots \rightsquigarrow\left(R+\delta S_{O(k n)}\right) \rightsquigarrow R$ works in the same fashion to achieve the same unique equilibrium.

\subsection{Manipulation Grammars}

The development of devices that generate programmable vector fields is still in its infancy. The existing prototype devices exhibit only a limited range of programmability. For example, the prototype MEMS arrays described in Section 2.1 currently have actuators in only four different directions, and the actu- ators are only row-wise controllable. Arrays with individually addressable actuators at various orientations are possible (Böhringer et al. 1994a, 1994b; Liu and Will 1995; Böhringer, Donald, and MacDonald 1996b; Suh et al. 1996) but require significant development effort. There are also limitations on the resolution of the devices given by fabrication constraints. For the vibrating-plate device from Section 2.2, the fields are even more constrained by the vibrational modes of the plate.

We are interested in the capabilities of such constrained systems. In this section, we give an algorithm that decides whether a part can be uniquely positioned using a given set of vector fields, and it synthesizes an optimal-length strategy if one exists. Furthermore, in Section 6.2, the vector fields we consider may be arbitrary, and in particular can vary in magnitude (as opposed to unit-squeeze fields). If we think of these vector fields as a vocabulary, we obtain a language of manipulation strategies. We are interested in those expressions in the language that corresponds to a strategy for uniquely posing the part.

We define two basic operations on vector fields. Consider two vector fields $f$ and $g: f+g$ denotes point-wise addition, and $f * g$ denotes sequential execution of $f$ and then $g$.

DEFINITION 13. Let $P$ be an arbitrary planar part. A finite field operator is a sequence of vector fields that brings $P$ from an arbitrary initial pose into a finite set of equilibrium poses.

A field operator comes with the following guarantee: no matter where in $\mathbb{R}^{2} \times \mathbb{S}^{1}$ the part starts off, it will always come to rest in one of $E$ different total equilibria (Fig. 22). That is, for any polygonal part $P$, either of these field operators is always guaranteed to reduce $P$ to a finite set of equilibria in its configuration space $\mathcal{C}=\mathbb{R}^{2} \times \mathbb{S}^{1}$.

From Section 6.1, we know that combined radial squeeze patterns $R+\delta S$ have this property. However, there are other simple field operators that also have this finiteness property.

ClAIM 1. Let $f$ and $f_{\perp}$ be unit-squeeze fields such that $f_{\perp}$ is orthogonal to $f$. Then the fields $f * f_{\perp}$ and $f+f_{\perp}$ induce a finite number of equilibria on every connected polygon $P$; hence $f * f_{\perp}$ and $f+f_{\perp}$ are finite field operators.

Proof. First, consider the field $f * f_{\perp}$, and without loss of generality assume that $f(x y)=(-\operatorname{sign}(x), 0)$. Also assume that the COM of $P$ is the reference point used to define its configuration space, $\mathcal{C}=\mathbb{R}^{2} \times \mathbb{S}^{1}$. As discussed in Sections 3.2 and 3.3, $P$ will reach one of a finite number of orientation equilibria when placed in $f$ or $f_{\perp}$. More specifically, when $P$ is placed in $f$, there exists a finite set of equilibria $E_{f}=$ $\left\{\left(x_{i}, \theta_{i}\right)\right\}$, where $x_{i}$ is the offset from $f$ 's squeeze line, and $\theta_{i}$ is the orientation of $P$ (see Section 3.4). Similarly for $f_{\perp}(x, y)=(0,-\operatorname{sign}(y))$, there exists a finite set of equilibria $E_{f_{\perp}}=\left\{\left(y_{j}, \theta_{j}\right)\right\}$. Since the $x$-component of $f_{\perp}$ is zero, the $x$-coordinate of the reference point of $P$ (the COM) remains constant while $P$ is in $f_{\perp}$. Hence $P$ will finally come to rest in a pose $\left(x_{k}, y_{k}, \theta_{k}\right)$, where $x_{k} \in \pi_{1}(E f),\left(y_{k}, \theta_{k}\right) \in E_{f_{\perp}}$, and 


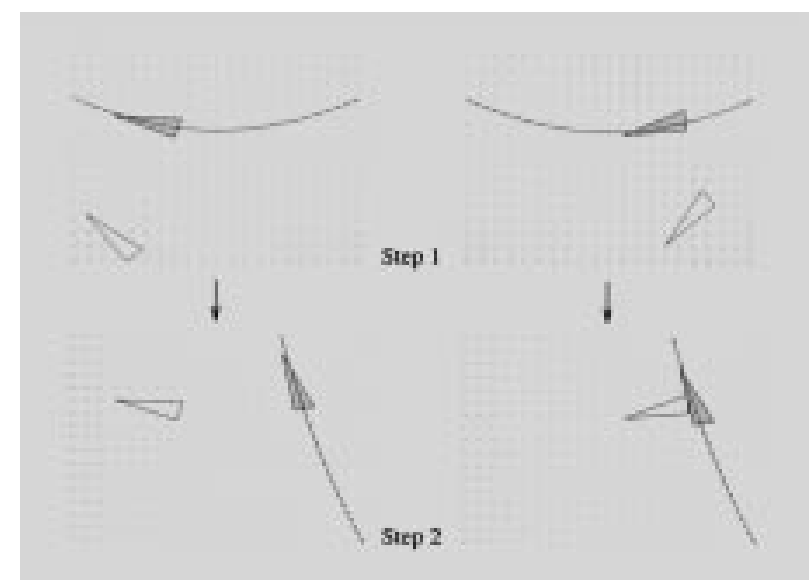

Fig. 22. Manipulation vocabulary for a triangular part on a vibrating plate, consisting of two consecutive force fields with slightly curved nodal lines (attractors), which bring the part into (approximately) the same equilibria.

$\pi_{1}$ is the canonical projection such that $\pi_{1}(x, \theta)=x$. Since $E f$ is finite, so is $\pi_{1}\left(E_{f}\right) . E\left(f_{\perp}\right)$ is also finite; therefore, there exists only a finite number of such total equilibrium poses for $f * f_{\perp}$.

If $P$ is placed into the field $f+f_{\perp}$, there exists a unique translation equilibrium for every given, fixed orientation $\theta$. In each of these translation equilibria, the squeeze lines of $f$ and $f_{\perp}$ are both bisectors of $P$. Now consider the moment acting on $P$ when $P$ is in translation equilibrium as a function of $\theta$. Since there are $O\left(n^{2}\right)$ topological placements for a single bisector, therefore there exist also only $O\left(n^{2}\right)$ topological placements for two simultaneous, orthogonal bisectors. In analogy to Proposition 1 in Section 3.2, we can show that for an topological placement of the bisectors, this moment function has at most $O(k)$ roots, where $k$ is the maximum number of edges a bisector of $P$ can cross. This implies that there exist only $O\left(k n^{2}\right)$ distinct total equilibria for $f+f_{\perp}$.

If we can assume that the 2 Phase assumption holds, then Claim 1 leads to an interesting extension of the parts-orienting algorithm described in Section 3.3. Let $f_{x}$ and $f_{y}$ be two orthogonal squeeze fields with their squeeze lines coinciding with the $x$-axis and the $y$-axis, respectively. Note that $f_{x} * f_{y}$ is a finite field operator. Let us append $f_{x} * f_{y}$ to an orienting strategy, $s$. After $s$ has been executed, the part will be uniquely orientated. Assuming that the 2Phase assumption holds, $f_{x} * f_{y}$ first brings the $y$-coordinate and then the $x$ coordinate of the part to a unique value, while maintaining unique orientation. Hence, given an arbitrary parts-orienting strategy $s$, by executing $s * f_{x} * f_{y}$, we obtain unique positioning and orienting. 6

6. This scheme can be simplified even further: Let $s_{n}$ be the last squeeze of the orienting strategy $s$. Let $s_{n}^{\perp}$ be a squeeze field orthogonal to $s_{n}$. Then it is easy to show that $s * s_{n}^{\perp}$ uniquely positions and orients the part.
COROLlaRY 6. Let $f$ be a finite field operator for a part $P$, and let $g$ be an arbitrary vector field. Then the sequence $g * f$ is a finite field operator.

Proof. By definition of a finite field operator, $f$ brings the part $P$ into a finite set of equilibrium poses from arbitrary initial poses, in particular, from the poses that are the result of field $g$.

Thus, by pre-pending an arbitrary sequence of fields to a finite field operator, one can always create a new finite field operator (possibly with a smaller set of discrete equilibria). In the remainder of this section, however, we will only consider finite field operators of minimal length, i.e., field sequences from which no field can be removed without losing the finiteness property (Definition 13).

We have seen in Sections 3 and 5 that for simple force fields such as squeeze or radial fields, we can predict the motion and the equilibria of a part using exact analytical methods. However, for arbitrary fields (e.g., the force fields described in Section 2.2, which are induced by vibrating plates), such algorithms are not known. Instead, we can employ approximate methods to predict the behavior of the part in the force field. These methods are typically numerical computations that involve simulating the part from a specific initial pose, until it reaches equilibrium. ${ }^{7}$ We call the cost for such a computation the simulation complexity $s(n)$. We write $s(n)$, because the simulation complexity will usually depend on the complexity of the part, i.e., its number of vertices $n$ (for more details also see the work of Donald and Xavier (1995)).

Proposition 14. Consider a polygonal part $P$, and $m$ finite field operators $\left\{F_{i}\right\}, 1 \leq i \leq m$, each with at most $E$ distinct equilibria in the configuration space $C$ for $P$. There exists an algorithm that generates an optimal length strategy of the form $F_{1} * F_{2} * \cdots * F_{l}$ to uniquely pose $P$ up to symmetries, if such a strategy exists. This algorithm runs in $O\left(m^{2} E\left(s(n)+2^{E}\right)\right)$ time, where $s(n)$ is the simulation complexity of $P$ in $F_{i}$. If no such strategy exists, the algorithm will signal failure.

Proof. Construct a transition table $T$ of size $m^{2} E$ that describes how the part $P$ moves from an equilibrium of $F_{i}$ to an equilibrium of $F_{j}$. This table can be constructed either by a dynamic analysis similar to that in Section 3.1, or by dynamic simulation. The time to construct this table is $O\left(m^{2} E s(n)\right)$, where $s(n)$ is the simulation complexity, which will typically depend on the complexity $n$ of the part.

Using the table $T$, we can search for a strategy as follows: define the state of the system as the set of possible equilibria a part is in, for a particular finite field operator $F_{i}$. There are $O(E)$ equilibria for each finite field operator; hence, there are $O\left(m 2^{E}\right)$ distinct states. For each state, there are $m$ possible

\footnotetext{
7. See, for example, the World Wide Web at www.ee.washington.edu/faculty/
} karl/Research/. 
successor states, as given by table $T$, and they can each be determined in $O(E)$ operations, which results in a graph with $O\left(m 2^{E}\right)$ nodes, $O\left(m^{2} 2^{E}\right)$ edges, and $O\left(m^{2} E 2^{E}\right)$ operations for its construction. Finding a strategy, or deciding that one exists, then devolves to finding a path whose goal node is a state with a unique equilibrium. The total running time of this algorithm is $\left.O\left(m^{2} E(s(n))+2^{E}\right)\right)$.

Hence, as discussed by Erdmann and Mason (1988), for any part we can decide whether a part can be uniquely posed using the vocabulary of field operators $\left\{F_{i}\right\}$, but (1) the planning time is worst-case exponential, and (2) we do not know how to characterize the class of parts that can be oriented by a specific family of operators $\left\{F_{i}\right\}$. However, the resulting strategies are optimal in length.

Manipulation grammars are discussed in much greater detail by Böhringer and colleagues (forthcoming) in the context of the limited manipulation vocabulary generated by vibrating plates.

\section{Conclusions and Open Problems}

The following table 1 summarizes fields and algorithms for manipulation tasks with programmable force fields, and includes some additional recent results.

Less-difficult tasks such as translation can be achieved with relatively simple fields and without any planning. More complex tasks, such as centering or unique orienting, require increasingly complex fields. However, planning complexity is, e.g., higher for sequences of squeeze fields, and lower for the more complex combined radial + squeeze fields. This illustrates a trade-off between mechanical complexity (the dexterity and controllability of actuator-array elements) and computational complexity (the algorithmic difficulty of synthesizing a strategy). For example, if one is willing to build a device capable of radial fields, then one reaps great benefits in planning and execution speed. On the other hand, we can still plan for simpler devices, but the plan synthesis is more expensive (worst-case exponential in the number of equilibria), and we lose some completeness properties.

We believe that the rapid growth in this research area will continue. Even though a science base for manipulation with programmable force fields has emerged, many important questions remain open. Some topics for future work are listed in the following paragraphs.

- Universal feeder-orienter (UFO) devices. It was shown in Proposition 1 that every connected polygonal part $P$ with $n$ vertices has a finite number of stable orientation equilibria when $P$ is placed into a squeeze field $S$. Based on this property, we were able to generate manipulation strategies for unique part alignment. We showed in Section 6.1 that by using a combined radial and squeeze field $R+\delta S$, the number of equilibria can be reduced to $O(k n)$. Using elliptic force fields $f(x, y)=(\alpha x, \beta y)$ such that $\alpha \neq \beta$ and $\alpha, \beta \neq 0$, this bound can be reduced to two (Kavraki 1995, 1997).

In

a stable equilibrium, the part's major principal axis of inertia lines up with the squeeze line to minimize the second moment of inertia.

Does there exist a universal field that, for every part $P$, has only one unique equilibrium (up to part symmetry)? Such a field could be used to build a universal parts feeder (Abell and Erdmann 1996) that uniquely positions a part without the need of a clock, sensors, or programming.

We propose a combined radial and "gravitational" field $R+\delta G$ that might have this property. $\delta$ is a small positive constant, and $G$ is defined as $G(x, y)=(0,-1)$. This device design is inspired by the "universal gripper" described by Abell and Erdmann (1996). Such a field could be obtained from a MEMS array that implements a unit radial force field. Instead of rectangular actuators in a regular grid, triangular actuators could be laid out in a polar-coordinate grid. The array could then be tilted slightly to obtain the gravity component; hence, such a device would be relatively easy to build. Alternatively, a resonating speaker, or a vibrating diskshaped plate that is fixed at the center, might be used to create a radial force field. Extensive simulations show that for every part we have tried, one unique total equilibrium is always obtained. We are working toward a rigorous proof of this experimental observation.

- Abstraction barriers. We believe that programmable force fields can be used as an abstraction barrier between parts positioning and feeding applications and devices implementing the requisite mechanical twodimensional force fields (MEMS arrays, vibratory devices, or other devices). That is, applications such as parts feeding can be formulated in terms of the force fields required. This then serves as a specification that the underlying device technology must deliver. Conversely, the capabilities of MEMS-array or vibratorydevice technology can be formulated in terms of the force fields they can implement. This means that device designers can potentially ignore certain details of the application process, and instead focus on matching the required force-field specification. This would free application engineers from needing to know much about process engineering, in the same way that software and algorithm designers often abstract away from details of the hardware. Such an abstraction barrier could permit hierarchical design, and allow application designs with greater independence from the underlying device technology. 
Table 1.

\begin{tabular}{|c|c|c|c|c|}
\hline \multirow[b]{2}{*}{ Task } & \multirow[b]{2}{*}{ Field(s) } & \multicolumn{3}{|c|}{ Complexity } \\
\hline & & Fields & Planning & Plan Steps \\
\hline Translate & Constant & $\begin{array}{l}\text { Constant magnitude } \\
\text { and direction }\end{array}$ & - & 1 \\
\hline \multirow[t]{2}{*}{ Center } & Radial & $\begin{array}{l}\text { Constant magnitude, } \\
\text { continuous directions }\end{array}$ & - & 1 \\
\hline & $\begin{array}{l}\text { Orthogonal squeezes } \\
\text { magnitude and direction }\end{array}$ & Piecewise constant & $O(1)$ & $O(1)$ \\
\hline \multirow[t]{2}{*}{ Uniquely orient } & Sequence of squeezes & $\begin{array}{l}\text { Piecewise constant } \\
\text { magnitude and direction }\end{array}$ & $O\left(k^{2} n^{4}\right)$ & $O\left(k n^{2}\right)$ \\
\hline & Inertial & $\begin{array}{l}\text { Smooth magnitude } \\
\text { piecewise-constant direction }\end{array}$ & $O(1)$ & $O(1)$ \\
\hline \multirow[t]{4}{*}{ Uniquely pose } & Manipulation grammar & $\begin{array}{l}m \text { arbitrary fields, } \\
\text { at most } E \text { stable equations }\end{array}$ & $O\left(m^{2} 2^{E}\right)$ & $\begin{array}{l}O\left(m 2^{E}\right) \\
\text { (not com- } \\
\text { plete) }\end{array}$ \\
\hline & Sequence of radial + squeeze & $\begin{array}{l}\text { Piecewise-continuous } \\
\text { magnitude and direction }\end{array}$ & $O\left(k^{2} n^{2}\right)$ & $O(k n)$ \\
\hline & Elliptic & Smooth magnitude and direction & $O(1)$ & $O(1)$ \\
\hline & UFO & Continuous magnitude and direction & -- & 1 \\
\hline
\end{tabular}

- Magnitude control. Consider an array in which the magnitude of the actuator forces cannot be controlled. Does there exist an array with constant magnitude in which all parts reach one unique equilibrium? Or can one prove that, without magnitude control, the number of distinct equilibria is always greater than one?

- Geometric filters. This paper focuses mainly on sensorless manipulation strategies for unique positioning of parts. Another important application of programmable vector fields are geometric filters, which would be useful for the sorting and singulation of parts. Figure 1 shows a simple filter that separates smaller and larger parts. We are interested in the question, Given $n$ parts, does there exist a vector field that will separate them into specific equivalence classes? For example, does there exist a field that moves small and large rectangles to the left, and triangles to the right? In particular, it would be interesting to know whether for any two different parts there exists a sequence of force fields that will separate them.

- Force-field computers. In this paper, we have demonstrated that even with a rather limited vocabulary of simple force fields, useful and quite complex tasks such as sensorless posing or sorting of parts can be performed. It might be possible that force fields could be used to solve certain classes of problems, by encoding them in particular force fields, part shapes, and initial and goal poses, resulting in a "force-field computer" that provides a physical implementation of the problem. Identifying the class of encodable problems might yield deeper insights into the complexity of parts manipulation with force-vector fields.

- Performance measures. Are there performance measures for how fast (in real time) an array will orient a part? In some sense, the actuators are fighting each other (as we have observed experimentally) when the part approaches equilibrium. For squeeze grasps, one measure of "efficiency," albeit crude, might be the integral of the magnitude of the moment function, i.e., $\int_{0}^{2 \pi}|M(\theta)| d \theta$. The issue is that if, for many poses, $|M(\theta)|$ is very small, then the orientation process will be slow. Better measures are also desirable.

- Uncertainty. In practice, neither the force-vector field nor the part geometry will be exact, and both can only be characterized up to tolerances (Donald 1989). This is particularly important at the microscopic scale. Within the framework of potential fields, we can express this uncertainty by considering not one single potential function $U_{P}$, but rather families of potentials that correspond to different values within the uncertainty range. Bounds on part and force tolerances will correspond to limits on the variation within these function families. An investigation of these limits will allow us to obtain upper error bounds for manipulation tasks under which a specific strategy will still achieve its goal.

A family of potential functions is a set $\left\{U_{\alpha}: \mathcal{C} \rightarrow\right.$ $\mathbb{R}\}_{\alpha \in J}$ where $J$ is an index set. For example, we may start with a single potential function $U: \mathcal{C} \rightarrow \mathbb{R}$ and define a family of potential functions $\mathcal{F}(U, \epsilon, z)$ as $\left\{\left\{U_{\alpha}: \mathcal{C} \rightarrow \mathbb{R} \mid\left\|U_{\alpha}(p)-U(p)\right\|_{z}<\epsilon\right\}\right.$ for some $\epsilon$ and 
norm $z$. This is analogous to defining a neighborhood in function space, using, e.g., the compact-open topology.

When we differentiate a family of potential fields (using the gradient), we obtain a differential inclusion instead of a differential equation. So if $\mathcal{F}(u)=\mathcal{F}(u, \epsilon, z)$, then $\nabla \mathcal{F}(u)=\left\{\nabla U_{\alpha}\right\}_{\alpha \in J}$

When considering families of potentials, the equilibrium may be known to lie only within a set $E_{i}$, although we may know that it is always a point in $E_{i}$. If the sets $E_{i}$ are of a small diameter less than some $\epsilon>0$, our algorithms could be extended to handle the $\epsilon$-approximations.

As a more general approach, we propose an algorithm based on back-projections: for a given part, let $B_{F_{i}}(G) \subset \mathcal{C}=\mathbb{R}^{2} \times \mathbb{S}^{1}$ be the back-projection (LozanoPérez, Mason, and Taylor 1984) of the set $G$ under $F_{i}$, where $G \subset \mathcal{C}$, and $F_{i}$ is a family of fields on $\mathbb{R}^{2}$. Then we wish to calculate a sequence of fields $F_{l}, F_{2}, \ldots, F_{k}$, such that $B_{F_{1}}\left(B_{F_{2}}\left(\cdots B_{F_{k}}(G) \cdots\right)\right)=$ $\mathcal{C}$, where $G$ is a single point in $\mathcal{C}$ (cf. Lozano-Pérez, Mason, and Taylor 1984; Erdmann and Mason 1988; Brost 1988; Donald 1989; Brigg 1992).

- Output sensitivity. We have seen in Sections 3.1, 6.1, and 6.2 that the efficiency of planning and executing manipulation strategies critically depends on the number of equilibrium configurations. Expressing the planning and execution complexity as a function of the number of equilibria $E$, rather than the number of vertices $n$, is called output-sensitive analysis. In practice, we have found that there are almost no parts with more than two distinct (orientation) equilibria, even in squeeze fields. This is far less than the $E=O\left(k n^{2}\right)$ upper bound derived in Section 3.2. If this observation can be supported by an exact or even statistical analysis of part shapes, it could lead to extremely good expected bounds on plan length and planning time, even for the less powerful strategies employing manipulation grammars (note that the complexity of the manipulation grammar algorithm in Proposition 14 is output-sensitive).

- Discrete force fields. For the manipulation strategies described in this paper, we assume that the force fields are continuous, i.e., that the generated forces are dense compared to the moving part (the density assumption in Section 3.3). When manipulating very small parts on microactuator arrays, this condition may be only approximately satisfied. We are interested in the limitations of the continuous model, and we would like to know the conditions under which it is necessary to employ a different, discrete model of the array that takes into account individual actuators, as well as the gaps between actuators. In the work of Böhringer et al. (1994b), we propose a model for the interaction between parts and arrays of individual actuators, based on the theory of limit surfaces (Goyal and Ruina 1988; Goya, Ruina, and Papadopoulos 1991).

- Resonance properties. Is it possible to exploit the dynamic resonance properties of parts to tune the control signal of the array or plate to perform efficient dynamic manipulation?

- 3-D force fields. It may be possible to generate 3-D force fields by using Lorentz electromagnetic forces. Tunable electric coils could be attached to various points of a 3-D body, suspending the resulting object in a strong permanent magnetic field using magnetic levitation (the Lorentz effect) (Hollis and Salcudean 1993; Salcudean, Wong, and Hollis 1995. The tuning (control) of the electric coils could be effected as follows: integrated control circuitry could be fabricated and colocated with the coils, and conceivably, a power supply. The control could be globally effected using wireless communication, or the control of each coil could evolve in time until the part is reoriented as desired. The Lorentz forces could then be deactivated to bring the object to rest on the ground. Planning for such a 3-D device might reduce to that described by Erdmann and colleagues (1993).

\section{Acknowledgments}

We would like to thank Tamara Lynn Abell, Vivek Bhatt, John Canny, Bernard Chazelle, Paul Chew, Perry Cook, Ken Goldberg, Al Rizzi, Ivelisse Rubio, Andy Ruina, Ken Steiglitz, Nick Trefethen, and Andy Yao for useful discussions and valuable comments. We are particularly grateful to Mike Erdmann for his suggestions and help for the proofs of Proposition 7 and Lemma 4, to Danny Halperin for sharing his insights into geometric and topological issues related to manipulation with force fields, to Lydia Kavraki for continuing discussions and creative new ideas concerning force-vector fields, and Jean-Claude Latombe for his hospitality during our stay at the Stanford Robotics Laboratory. We thank the staff, students, and users of the Cornell Nanofabrication Facility for their help and support.

Support was provided in part by the NSF under grants IRI-8802390, IRI-9000532, IRI-9201699, IRI-9530785, and CISE/CDA 98-05548 IRI-9896020, and by a Presidential Young Investigator award to Bruce Donald, in part by NSF/ARPA Special Grant for Experimental Research IRI9403903, and in part by the AFOSR, the Mathematical Sciences Institute, Intel Corporation, and AT\&T Bell Laboratories. This work was supported by ARPA under contract DABT 63-69-C-0019. The microactuator fabrication was performed 
at the Cornell Nanofabrication Facility (CNF), which is supported by NSF grant ECS-8619049, Cornell University, and Industrial Affiliates.

\section{References}

Abell, Tamara Lynn, and Erdmann, Mike. 1996. A universal parts feeder. Personal communication.

Akella, Srinivas, Huang, Wesley H., Lynch, Kevin M., and Mason, Matthew T, 1995. Planar manipulation on a conveyor b a one-joint robot with and without sensing. Int. Symp. of Robot. Res. (ISSR).

Analog Devices, Inc. 1991. Introducing the ADXL50 Micromachined Accelerometer Sensor. Norwood, Massachusetts.

Böhringer, Karl-Friedrich, Bhatt, Vivek, Donald, Bruce R., and Goldberg, Kenneth Y. (forthcoming). Sensorless manipulation using transverse vibrations of a plate. Algorithmica, Special Issue on Algorithmic Foundations of Robotics.

Böhringer, Karl-Friedrich, Bhatt, Vivek, and Goldberg, Kenneth Y. 1995 (May, Nagoya, Japan). Sensorless manipulation using transverse vibrations of a plate. Proc. of the IEEE Int. Conf. on Robot. and Automat. (ICRA). Washington, DC: IEEE, pp. 1989-1996. Also available on the Web at http://www.cs.cornell.edu/home/karl/VibratoryAlign.

Böhringer, Karl-Friedrich, Brown, Russell G., Donald, Bruce R., Jennings, James S., and Rus, Daniela. 1995 (June, Stanford, California). Distributed robotic manipulation: Experiments in minimalism. Experimental Robotics IV, vol. 223 of Lecture Notes in Control and Information Sciences. Berlin: Springer-Verlag, pp. 11-25.

Böhringer, Karl-Friedrich, Donald, Bruce R., and Halperin, Dan. 1997 (June, Nice). The area bisectors of a polygon and force equilibria in programmable vector fields. Proc. of the 13th ACM Symp. on Computational Geometry. New York: ACM.

Böhringer, Karl-Friedrich, Donald, Bruce R., and MacDonald, Noel C. 1996a (February, San Diego, California). Single-crystal silicon actuator arrays for micromanipulation tasks. Proc. of the IEEE Workshop on Micro Electro Mechanical Systems (MEMS). Washington, DC: IEEE, pp. 7-12. Also available on the Web at http://www.cs.cornell.edu/home/karl/MicroActuators.

Böhringer, Karl-Friedrich, Donald, Bruce R., and MacDonald, Noel C. 1996b (July, Toulouse). Upper and lower bounds for programmable vector fields with applications to MEMS and vibratory plate parts feeders. Int. Workshop on Algorithmic Foundations of Robot. (WAFR). Also available on the Web at http://www.cs.cornell.edu/home/karl/MicroManipulation.

Böhringer, Karl-Friedrich, Donald, Bruce R., MacDonald, Noel C., Kovacs, Gregory T. A., and Suh, John W. 1997. Computational methods for design and control of MEMS micromanipulator arrays. Comp. Sci. Eng. (Jan./March):17-29.

Böhringer, Karl-Friedrich, Donald, Bruce R., Mihailovich, Robert, and MacDonald, Noel C. 1994a (May, San Diego, California). Sensorless manipulation using massively parallel microfabricated actuator arrays. Proc. of the IEEE Int. Conf. on Robot. and Automat. (ICRA). Los Alamitos, California: IEEE, pp. 826-833. Also available on the Web at http://www.cs.cornell.edu/home/karl/MicroManipulation.

bßöhringer, Karl-Friedrich, Donald, Bruce R., Mihailovich, Robert, and MacDonald, Noel C. 1994b (January, Oiso, Japan). A theory of manipulation and control for microfabricated actuator arrays. Proc. of the IEEE Workshop on Micro Electro Mechanical Systems (MEMS). Los Alamitos, California: IEEE, pp. 102-107. Also available on the Web at http://www.cs.cornell.edu/home/karl/MicroActuators.

Böhringer, Karl-Friedrich, Suh, John W., Donald, Bruce R., and Kovacs, G. T. A. 1997 (April, Albuquerque, New Mexico). Vector fields for task-level distributed manipulation: Experiments with organic micro actuator arrays. Proc. of the IEEE Int. Conf. on Robot. and Automat. (ICRA). Washington, DC: IEEE, pp. 1779-1786.

Boothroyd, Geoffrey, Poli, Corrado, and Murch, Laurence E. 1982. Automatic Assembly. Marcel-Dekker.

Briggs, Amy J. 1992. An efficient algorithm for one-step planar compliant motion planning with uncertainty. Algorithmica 8(3).

Brooks, Rod 1986. A layered intelligent control system for a mobile robot. IEEE J. Robot. Automat. RA(2).

Brost, Randy C. 1988. Automatic grasp planning in the presence of uncertainty. Int. J. Robot. Res. 7(1):3-17.

Canny, John, and Goldberg, Ken 1994. "RISC" for industrial robotics: Recent results and open problems. Proc. IEEE Int. Conf. on Robot. and Automat. (ICRA). Los Alamitos, CA: IEEE.

Darling, Bruce R., Suh, John W., and Kovacs, Gregory T. A. 1997 (October 20-24, San Jose, California). Ciliary microactuator array for scanning electron microscope positioning stage. Proc. of the AVS 44th National Symp.

Diaz, M., and O'Rourke, J. 1990 (Ottawa). Ham-sandwich sectioning of polygons. Proc. of the 2nd Canadian Conf. on Computational Geometry, pp. 95-101.

Donald, Bruce R. 1989. Error Detection and Recovery in Robotics, vol. 336 of Lecture Notes in Computer Science. Berlin: Springer-Verlag.

Donald, Bruce R. 1990. The complexity of planar compliant motion planning with uncertainty. Algorithmica 5(3):353382.

Donald, Bruce Randall, Jennings, James, and Rus, Daniela. 1995. Information invariants for distributed manipulation. Int. Workshop on Algorithmic Found. of Robot. (WAFR). Wellesley, Massachusetts: A. K. Peters, pp. 431-459.

Donald, Bruce Randall, and Xavier, Pat. 1995. Provably good approximation algorithms for optimal kinodynamic 
planning for Cartesian robots and open-chain manipulators. Algorithmica 14(6):480-630.

Erdmann, M. A. 1994. On a representation of friction in configuration space. J. Robot. Res. 13(3):240-271.

Erdmann, Michael A. 1996. An exploration of nonprehensile two-arm manipulation: Planning and execution. Technical Report, Carnegie Mellon University, Pittsburgh, Pennsylvania.

Erdmann, Michael A., and Mason, Matthew T. 1988. An exploration of sensorless manipulation. IEEE J. Robot. Automat. 4(4).

Erdmann, Michael A., and Mason, Matthew T. 1996 (July, Toulouse). Nonprehensile manipulation. Int. Workshop on Algorithmic Found. of Robot. (WAFR). Wellesley, Massachusetts: A. K. Peters.

Erdmann, Michael A., Mason, Matthew T., and Vaneček, Jr., G. 1993. Mechanical parts orienting: The case of a polyhedron on a table. Algorithmica 10.

Farnum, Gregory T., and Davis, Bill. 1986. Delivering the part. Manufact. Eng. (March).

Fujita, Hiroyuki. 1993 (October 26-28, Tokyo). Group work of microactuators. Int. Adv. Robot Program Workshop on Micromachine Tech. and Sys., pp. 24-31.

Gabriel, Kaigham J. 1995. Engineering microscopic machines. Sci. Am. 273(3).

Gillmer, Thomas 0. 1956. Fundamentals of Construction and Stability of Naval Ships. Annapolis, Maryland: U. S. Naval Institute.

Goldberg, K. Y. 1993. Orienting polygonal parts without sensing. Algorithmica 10(2/3/4):201-225.

Goyal, Suresh, and Ruina, Andy. 1988. Relation between load and motion for a rigid body sliding on a planar surface with dry friction: Limit surfaces, incipient and asymptotic motion. Wear.

Goyal, Suresh, Ruina, Andy, and Papadopoulos, Jim. 1991. Planar sliding with dry friction. Part 1: Limit surface and moment function. Part 2: Dynamics of motion. Wear 143:307-352.

Hitakawa, Hajime. 1988. Advanced parts orientation system has wide application. Assembly Automation 8(3).

Hollis, Ralph, and Salcudean, S. E. 1993 (October 1-3, Hidden Valley, Pennsylvania). Lorentz levitation technology: A new approach to fine-motion robotics, teleoperation, haptic interfaces, and vibration isolation. Int. Symp. on Robot. Res. (ISRR).

Jacobson, J. D. Goodwin-Johansson, S. H., Bobbio, S. M., Bartlet, C. A., and Yadon, N. 1995. Integrated force arrays: Theory and modeling of static operation. J. Microelectromechanical Sys. 4(3):139-150.

Kavraki, Lydia E. 1995. On the number of equilibrium placements of mass distributions in elliptic potential fields. Technical Report STAN-CS-TR-95.1559, Department of Computer Science, Stanford University, Stanford, California 94305.
Kavraki, Lydia. 1997 (April, Albuquerque, New Mexico). Part orientation with programmable vector fields: Two stable equilibria for most parts. Proc. IEEE Int. Conf. on Robot. and Automat. (ICRA). Washington, DC: IEEE.

Khatib, Oussama. 1986. Real-time obstacle avoidance for manipulators and mobile robots. Int. J. Robot. Res. 5(1):90-99.

Koditschek, Daniel E., and Rimon, Elon. 1988. Robot navigation functions on manifolds with boundary. Adv. Appl. Math.

Konishi, Satoshi, and Fujita, Hiroyuki. 1993 (March, Kawasaki, Japan). A proposal for a conveyance system with autonomous decentralized micro modules. Proc. of the IEEE Int. Sym. on Autonomous Decentralized Sys. Los Alamitos, California: IEEE.

Liu, Wenheng, and Will, Peter. 1995 (Pittsburgh, Pennsylvania). Parts manipulation on an intelligent motion surface. Proc. of the IEEE/RSJ Int. Workshop on Intell. Robots and Sys. (IROS). Washington, DC: IEEE.

Lozano-Pérez, Tomás. 1983. Spatial planning: A configurationspace approach. IEEE Trans. Comp. C32(2):108-120.

Lozano-Pérez, Tomás, Mason, Matt, and Taylor, Russell. 1984. Automatic synthesis of fine-motion strategies for robots. Int. J. Robot. Res. 3(1).

MacDonald, Noel C. Forthcoming. Nanostructures in motion:Microinstruments for moving nanometer-scale objects. In G. Timp (ed.): Nano-Science and Technology: A New Frontier. A\&P Press.

MacDonald, Noel C. 1996. SCREAM microelectromechanical systems. J. Microelectronic Eng. 32:49-73.

MacDonald, N. C., Adams, S. G. Ayon, A. A., Böhringer, K.F., Chen, L.-Y., Das, J. H., Haronian, D., Hofmann, W. Huang, X. T., Jazairy, A., Mihailovich, R. E., Miller, S. A., Ogo, I., Prasad, R., Reed, B. W., Saif, M. T. A., Shaw, K. A., Webb, R. Y., and Xu, Y. 1996. Microelectronic Eng. 30(1/4):563-564.

McGeer, T. 1990. Passive dynamic walking. Int. J. Robot. Res.

Mihailovich, R. E., and MacDonald, N. C. 1996. Dissipation measurements of vacuum-operated single-crystal silicon resonators. Sensors and Actuators.

Mihailovich, R. E., Zhang, Z. L., Shaw, K. A., and MacDonald, N. C. 1993 (February, Fort Lauderdale, Florida). Single-crystal silicon torsional resonators. Proc. of the IEEE Workshop on Micro-Electro Mechanical Systems (MEMS). Los Alamitos, California: IEEE, pp. 155-160.

Moncevicz, Paul, Jakiela, Mark, and Ulrich, Karl. 1991 (September). Orientation and insertion of randomly presented parts using vibratory agitation. Proc. of the $3 r d$ ASME Conf. on Flexible Assembly Sys. New York: ASME.

Nevins, James L., and Whitney, Daniel E. 1978. Computercontrolled assembly. Sci. Am.

Newman, John Nicholas. 1977. Marine Hydrodynamics. Cambridge, Massachusetts: MIT Press. 
Peterson, K. E. 1982. Silicon as a mechanical material. Proc. IEEE 70.

Pister, K. S. J., Fearing, R., and Howe, R. 1990 (February, Napa Valley, California). A planar air-levitated electrostatic actuator system. Proc. of the IEEE Workshop on $\mathrm{Mi}$ cro Electro Mechanical Sys.(MEMS). Los Alamitos, California: IEEE, pp. 67-71.

Raibert, M. H., Hodgins, J. K., Playter, R. R., and Ringrose, R. P. 1993. Animation of legged maneuvers: Jumps, somersaults, and gait transitions. J. Robot. Soc. Japan 11(3):333-341.

Reif, John, and Wang, H. 1995. Social potential fields: A distributed behavioral control for autonomous robots. In Goldberg, K., Halperin, D., Latombe, J.-C., and Wilson,R. (eds): International Workshop on Algorithmic Foundations of Robotics (WAFR). Wellesley, Massachusetts: A. K. Peters, pp. 431-459.

Riley, J. 1983. Assembly Automation, A Management Handbook. New York: Industrial Press.

Rimon, Elon, and Koditschek, Dan. 1992. Exact robot navigation using artificial potential functions. IEEE Trans. Robot. Automat. 8(5).

Saif, M. Taher A., and MacDonald, Noel C. 1995 (June, Stockholm). A milliNewton micro-loading device. Transducers---Digest Int. Conf. on Solid-State Sensors and Actuators, pp. 25-29.

Salcudean, S. E., Wong, N. M., and Hollis, R. L. 1995. Design and control of a force-reflecting teleoperation system with magnetically levitated master and wrist. IEEE Trans. Robot. Automat. 11(6).

Sampsell, J. B. 1993 (June, Yokahama). The digital micromirror device and its application to projection displays. Transducers---Digest Int. Conf. on Solid-State Sensors and Actuators, pp. 24-27.

Sandler, Ben-Zion. 1991. Robotics; Designing the Mechanisms for Automated Machinery. Englewood Cliffs, New
Jersey: Prentice Hall.

Schroer, Berhard J. 1987. Electronic parts presentation using vibratory bowl feeders. Robotics 3 .

Shaw, Kevin A., and MacDonald, Noel C. 1996 (February, San Diego, California). Integrating SCREAM micromechanical devices with integrated circuits. Proc. IEEE Workshop on Micro-Electro Mechanical Systems (MEMS). Washington, DC: IEEE.

Shaw, Kevin A., Zhang, Z. Lisa, and MacDonald, Noel C. 1993 (June 7-10, Yokahama). SCREAM I: A single mask, single-crystal silicon process for microelectromechanical structures. Transducers---Digest Int. Conf. on Solid-State Sensors and Actuators.

Singer, N. C., and Seering, W. P. 1987. Utilizing dynamic stability to orient parts. J. Appl. Mech. 54:961-966.

Storment, C. W., Borkholder, D. A., Westerlind, V., Suh, J. W., Maluf, N. I., and Kovacs, G. T. A. 1994. Flexible, dry-released process for aluminum electrostatic actuators. J. Microelectromechanical Sys. 3(3):90-96.

Suh, John W., Glander, Steven F., Darling, Robert B., Storment, Christopher W., and Kovacs, Gregory T. A.. 1996 (June, Hilton Head, North Carolina). Combined organic thermal and electrostatic omnidirectional ciliary microactuator array for object positioning and inspection. Proc. of the Solid-State Sensor and Actuator Workshop.

Xu, Yang, Miller, Scott A., and MacDonald, Noel C. 1995. Microelectromechanical scanning tunneling microscope. Bull. Am. Physical Soc. 40(1):63.

Zhang, Z. Lisa, and MacDonald, Noel C. 1992. An RIE process for submicron, silicon electromechanical structures. J. Micromechanics Microengineering 2(1):31-38.

Zumel, A. B., and Erdmann, M. A. 1996 (April, Minneapolis, Minnesota). Nonprehensile two-palm manipulation with nonequilibrium transitions between stable states. Proc. of the IEEE Int. Conf. on Robot. and Automat. (ICRA). Washington, DC: IEEE. 